\title{
Modern vegetation proxies reflect Palaeogene and Neogene vegetation evolution and climate change in Europe, Turkey, and Armenia
}

\author{
Johanna Kovar-Eder, Petr Mazouch, Vasilis Teodoridis, Anita Roth-Nebelsick, \\ Christopher Traiser, and Janina Wypich
}

\begin{abstract}
Recently two tools, Drudge 1 and 2, were introduced to more easily assess modern vegetation proxies for the fossil record. They are based on three similarities: the Integrated Plant Record (IPR) Similarity assessing the similarity between fossil assemblages and modern vegetation based on the proportion of major zonal angiosperm components; the Taxonomic Similarity (TS) reflecting the similarity based on the coincidence of genera among the fossil record and modern vegetation; and the combination of both similarities (Results Mix). In this paper, we apply Drudge 1 and 2 to 54 fossil plant assemblages from Europe, Turkey, and Armenia covering the time span from the early Eocene to the Pleistocene. For every fossil plant assemblage, a set of 25 proxies (modern vegetation units) is derived by the Drudges, reflecting its physiognomy and floristic composition. The results for all fossil plant assemblages feature the overall vegetation evolution and climate change in Europe, Turkey, and Armenia providing deeper insight into the relationships of the fossil record to the modern Asian and European vegetation. This study strives to provide an improved understanding of the Palaeogene/Neogene vegetation evolution in Europe and Asia beyond the community of specialists. Our results can help model future scenarios. They also improve our understanding of how climate change may affect vegetation and more broadly ecosystems as a whole.
\end{abstract}

Johanna Kovar-Eder. State Museum of Natural History Stuttgart, Rosenstein 1, 70191 Stuttgart, Germany. johanna.eder@smns-bw.de

Petr Mazouch. Faculty of Informatics and Statistics, University of Economics, Prague, Czech Republic. mazouchp@vse.cz

Vasilis Teodoridis. Department of Biology and Environmental Studies, Faculty of Education, Charles University, Prague, Czech Republic. vasilis.teodoridis@pedf.cuni.cz

Anita Roth-Nebelsick. State Museum of Natural History Stuttgart, Rosenstein 1, 70191 Stuttgart, Germany. anita.rothnebelsick@smns-bw.de

Kovar-Eder, Johanna, Mazouch, Petr, Teodoridis, Vasilis, Roth-Nebelsick, Anita, Traiser, Christopher, and Wypich, Janina. 2021. Modern vegetation proxies reflect Palaeogene and Neogene vegetation evolution and climate change in Europe, Turkey, and Armenia. Palaeontologia Electronica, 24(2):a18. https://doi.org/10.26879/1131

palaeo-electronica.org/content/2021/3352-vegetation-evolution-and-climate-change

Copyright: May 2021 Paleontological Society.

This is an open access article distributed under the terms of Attribution-NonCommercial-ShareAlike 4.0 International (CC BY-NC-SA 4.0), which permits users to copy and redistribute the material in any medium or format, provided it is not used for commercial purposes and the original author and source are credited, with indications if any changes are made.

creativecommons.org/licenses/by-nc-sa/4.0/ 
Christopher Traiser. University Tübingen, Departement of Geoscience, Schnarrenbergstr. 94-96, 72076

Tübingen, Germany. christopher.traiser@uni-tuebingen.de

Janina Wypich. State Museum of Natural History Stuttgart, Rosenstein 1, 70191 Stuttgart, Germany.

janina.wypich@smns-bw.de

Key words: vegetation; climate; evolution; Palaeogene; Neogene; Eurasia

Submission: 7 October 2020. Acceptance: 23 April 2021.

\section{INTRODUCTION}

Vegetation is a key indicator for understanding Earth's evolution and climate change. The natural distribution as well as physiognomy of major vegetation types/formations is linked to climate, while aspects of climate can be influenced by the vegetation cover. Moreover, vegetation and its floristic composition are basic traits of any terrestrial ecosystem. Finally, flora and vegetation, and fauna are closely related and variously dependent on each other within one ecosystem. Accordingly, the assessment of the flora and vegetation along with their evolution over time is crucial for our understanding of general ecosystem evolution.

In Europe, the Palaeogene and Neogene plant record (both macro- and micro-fossils) is extremely rich and comparatively well studied within a stratigraphically well-defined framework. This offers an excellent baseline for assessing vegetation and (palaeo) climate evolution. For nonspecialists in plant sciences, however, it is a major challenge to understand the differences both in composition and ecological properties of different vegetation types. This paper aims to bridge this gap by assessing modern European and East Asian vegetation types that resemble assemblages in the European fossil record based on their physiognomic and taxonomic character (modern proxies).

In contrast to attempts for (palaeo) climate reconstruction and modelling, approaches to assess former large-scale vegetation are rare. The Integrated Plant Record (IPR) vegetation analysis is designed to assess major types of deep-time vegetation based on leaf physiognomy and the autecology of the fossil taxa of plant taphocoenoses, including a full range of plant organ assemblages (Kovar-Eder and Kvaček, 2003, 2007; Kovar-Eder et al., 2008). The concept of Utescher et al. (2007) applies plant functional types of trees to this end, but differs in substantial aspects from IPR vegetation analysis. It includes climatic parameters in the scoring groups, ignores herbaceous plants, and it does not differentiate between zonal and azonal elements. Other approaches to assess former vegetation focus more on regional aspects (Bouchal et al., 2018) or are restricted to the fossil record of specific plant organs such as the diaspore record (Martinetto and Vassio, 2010).

The IPR vegetation analysis was tested on modern vegetation assemblages from China and Japan (Teodoridis et al., 2011, 2012). Most recently, two mathematical tools (Drudge 1 and Drudge 2) were introduced to more easily determine modern vegetation proxies (Teodoridis et al., 2020). Modern proxies are important because they facilitate our understanding of past vegetation properties. Drudge 1 and Drudge 2 are based on the IPR vegetation analysis, which was extended for the "Taxonomic Similarity" (TS) at the genus level. Moreover, the reference dataset of modern vegetation in Teodoridis et al. (2020) was considerably extended, including the vegetation from Europe and the Caucasus (Bohn et al., 2004) as well as from China (following Wang, 1961). Finally, in that paper Drudge 1 and 2 were tested on six fossil floras from Central Europe ranging from the late Eocene to the late Pliocene.

Here we present the comprehensive application of Drudge 1 and 2 to 54 fossil floras from Europe, Turkey, and Armenia ranging from the early Eocene to the Pleistocene. This more precisely renders both the floristic and the vegetation evolution in this region by modern proxies. We further demonstrate that the modern proxies identified by this methodology reflect overall climate change.

\section{MATERIAL}

Fifty-four assemblages of fossil plant remains (including leaf, carpological, and pollen record) from Europe, Turkey, and Armenia and ranging from the early Eocene to Pleistocene were analysed by the IPR vegetation analysis, and then Drudges 1 and 2 (Teodoridis et al., 2020) were applied (Figure 1, Table 1, Appendix 1). Nine of the assemblages comprise leaf, carpological, and pollen records, eight include leaves and carpological material, nine leaves and pollen, one fruit and pol- 


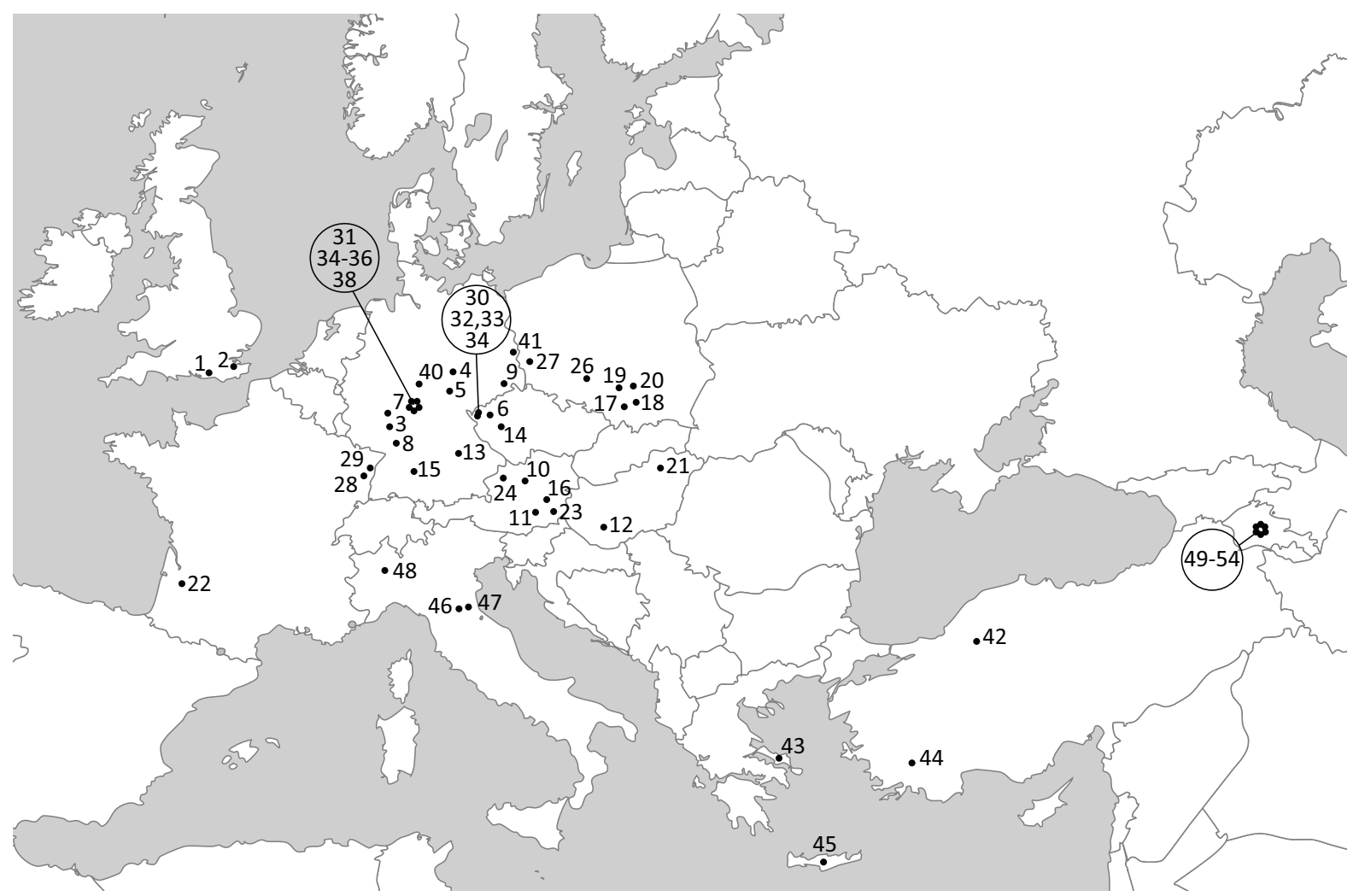

FIGURE 1. Geographic sketch showing the location of the plant-bearing sites. For locality numbers see Table 1.

len, 16 are leaf assemblages, and 11 are fruit assemblages. In selecting the assemblages, we considered the following criteria: diversity of taxa, reasonable taxonomic resolution, relevance for regional coverage, and stratigraphic control. The number of taxa per assemblage varied between 32 (Horní Břiza) and 204 (Wackersdorf), the mean was 101 taxa. The number of zonal taxa, which is the relevant parameter for applying the IPR vegetation analysis and the Drudges (see section Methods), varied from 16 (Nordhausen) to 151 (Arjuzanx) (mean: 68 zonal taxa).

\section{METHODS}

\section{Integrated Plant Record (IPR) Vegetation Analysis}

The IPR vegetation analysis is a semi-quantitative method that assesses major zonal vegetation types in deep time based on the fossil plant record - including the leaf, carpological, and pollen records (Kovar-Eder and Kvaček, 2003, 2007, Kovar-Eder et al., 2008). This approach is based on leaf physiognomy and the autecology of most similar living relatives. The proportions of major zonal angiosperm components are decisive for the assignment to a vegetation type. These major zonal angiosperm components are broad-leaved deciduous (BLD), broad-leaved evergreen (BLE), sclerophyllous (SCL), legume-like (LEG), dry herbs (DRY HERB), and mesophytic herbs (MESO HERB). The concept was evaluated by applying this approach to the modern vegetation in East Asia and was then adapted by Teodoridis et al. (2011). Since the plausibility of the results increases with the number of zonal taxa, the original minimum threshold of at least 10 zonal taxa was later raised to 15 (Kovar-Eder and Teodoridis, 2018).

\section{Drudge 1 and 2}

The objectives of the newly introduced tools, Drudges 1 and 2 (Teodoridis et al., 2020, 2021), are two-fold: (1) to facilitate the application of the IPR vegetation analysis, (2) to automatically assess proxies from the reference data set of modern vegetation units. They do this based on the similarity of the proportions of the major zonal angiosperms and the coincidence of the floristic 
TABLE 1. Fossil plant assemblages from Europe and Asia Minor (hereafter "test set of fossil assemblages") covering the period from the early Eocene to the Pleistocene, their ages, fossil record, and references. Country abbreviations. GB, Great Britain; DE, Germany; CZ, Czech Republic; AT, Austria; HU, Hungary; PL, Poland; FR, France; TY, Turkey; GR, Greece; IT, Italy; AM, Armenia.

\begin{tabular}{|c|c|c|c|c|c|c|c|c|c|c|c|}
\hline & 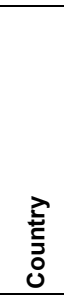 & 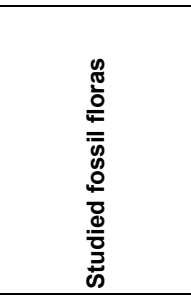 & $\begin{array}{l}\frac{-}{0} \\
\frac{2}{5} \\
i \frac{7}{4} \\
. \subseteq \\
0\end{array}$ & 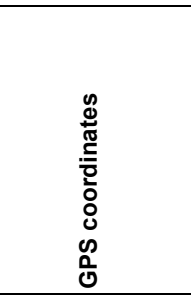 & 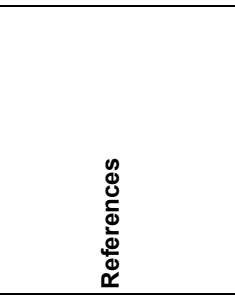 & $\stackrel{\mathscr{E}}{\frac{\mathscr{C}}{\infty}}$ & 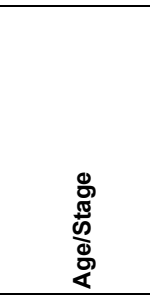 & 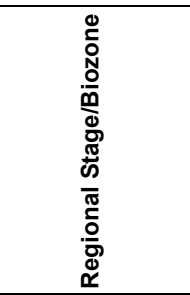 & $\sum^{\pi}$ & 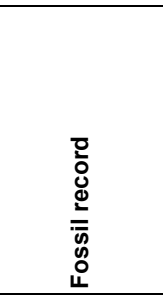 & 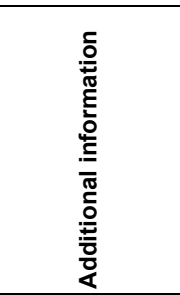 \\
\hline \multirow{16}{*}{ 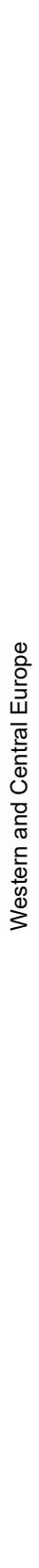 } & GB & $\begin{array}{l}\text { Hampshire } \\
\text { Basin }\end{array}$ & 1 & & Collinson (1983) & $\begin{array}{l}\text { early } \\
\text { Eocene }\end{array}$ & & & $56-49$ & fruits & \\
\hline & GB & London Clay & 2 & & Collinson (1983) & $\begin{array}{l}\text { early } \\
\text { Eocene }\end{array}$ & & & $56-49$ & fruits & \\
\hline & $\mathrm{DE}$ & Messel & 3 & $\begin{array}{l}\mathrm{N} 49^{\circ} 56^{\prime} \\
\mathrm{E} 8^{\circ} 44^{\prime}\end{array}$ & $\begin{array}{l}\text { Collinson et al. } \\
\text { (2012), Sturm } \\
\text { (1971), Wilde } \\
\text { (1989), Wilde et al. } \\
(2005)\end{array}$ & $\begin{array}{l}\text { early middle } \\
\text { Eocene }\end{array}$ & $\begin{array}{l}\text { lower } \\
\text { Geiselian }\end{array}$ & $\begin{array}{l}\text { Europ. } \\
\text { Vertebrate zone } \\
\text { MP } 11\end{array}$ & & leaves, fruits & \\
\hline & $\mathrm{DE}$ & Geiseltal & 4 & $\begin{array}{l}\text { N } 51^{\circ} 18^{\prime} 28^{\prime \prime} \\
\text { E } 11^{\circ} 52^{\prime} 09^{\prime \prime}\end{array}$ & $\begin{array}{l}\text { Mai (1976), Wilde } \\
\text { (1995), Kahlert and } \\
\text { Rüffle (2007) }\end{array}$ & $\begin{array}{l}\text { middle } \\
\text { Eocene }\end{array}$ & & & & leaves, fruits & \\
\hline & $\mathrm{DE}$ & $\begin{array}{l}\text { Profen- } \\
\text { Scheiplitz }\end{array}$ & 5 & $\begin{array}{l}\text { N } 51^{\circ} 7^{\prime} 32.45^{\prime \prime} \\
\text { E } 12^{\circ} 12^{\prime} 56.56^{\prime \prime}\end{array}$ & $\begin{array}{l}\text { Fischer (1991), Mai } \\
\text { and Walther (2000) }\end{array}$ & $\begin{array}{l}\text { late middle } \\
\text { Eocene }\end{array}$ & & & & leaves, fruits & \\
\hline & $C Z$ & Staré Sedlo & 6 & $\begin{array}{l}\text { N } 50^{\circ} 10^{\prime} 54.39^{\prime \prime} \\
\text { E } 12^{\circ} 43^{\prime} 11.37^{\prime \prime}\end{array}$ & $\begin{array}{l}\text { Knobloch et al. } \\
\text { (1996), Teodoridis } \\
\text { et al. (2012) }\end{array}$ & late Eocene & Priabonian & & $?$ & $\begin{array}{l}\text { leaves, pollen } \\
\text { (morphotaxa) }\end{array}$ & Staré Sedlo s.I. \\
\hline & $\mathrm{DE}$ & Flörsheim & 7 & $\begin{array}{l}\text { N } 50^{\circ} 01^{\prime} \\
\mathrm{E} 08^{\circ} 26^{\prime}\end{array}$ & Kvaček (2004) & $\begin{array}{l}\text { early } \\
\text { Oligocene }\end{array}$ & Rupelian & $\begin{array}{l}\text { Nannoplankton } \\
\text { zone NP23 }\end{array}$ & & leaves & \\
\hline & $\mathrm{DE}$ & Rauenberg & 8 & $\begin{array}{l}\text { N } 49^{\circ} 16^{\prime} 04^{\prime \prime} \\
\text { E } 8^{\circ} 42^{\prime} 13^{\prime \prime}\end{array}$ & Kovar-Eder (2016) & $\begin{array}{l}\text { early } \\
\text { Oligocene }\end{array}$ & Rupelian & $\begin{array}{l}\text { Nannoplankton } \\
\text { zone NP23 }\end{array}$ & & leaves & \\
\hline & $\mathrm{DE}$ & Seifhennersdorf & 9 & $\begin{array}{l}\text { N } 50^{\circ} 56^{\prime} 7.02^{\prime \prime} \\
\text { E } 14^{\circ} 36^{\prime} 26.99^{\prime \prime}\end{array}$ & $\begin{array}{l}\text { Walther and Kvaček } \\
(2007)\end{array}$ & $\begin{array}{l}\text { early } \\
\text { Oligocene }\end{array}$ & Rupelian & & $30.44 \pm 1.25$ & leaves & \\
\hline & AT & $\begin{array}{l}\text { Linz, Ebelsberg } \\
\text { Fm. }\end{array}$ & 10 & $\begin{array}{l}\text { N } 48^{\circ} 18^{\prime} \\
\text { E } 14^{\circ} 17^{\prime}\end{array}$ & Kovar (1982) & $\begin{array}{l}\text { early } \\
\text { Miocene }\end{array}$ & Aquitanian & $\begin{array}{l}\text { lowermost } \\
\text { upper Egerian, } \\
\text { nannoplankton } \\
\text { zone NN1 }\end{array}$ & app. 21.6-23 & leaves & \\
\hline & AT & Oberdorf & 11 & $\begin{array}{l}\text { N } 47^{\circ} 04^{\prime} \\
\text { E } 15^{\circ} 07^{\prime}\end{array}$ & $\begin{array}{l}\text { Kovar-Eder et al. } \\
(2001)\end{array}$ & $\begin{array}{l}\text { early } \\
\text { Miocene }\end{array}$ & $\begin{array}{l}\text { upper } \\
\text { Burdigalian }\end{array}$ & $\begin{array}{l}\text { Ottnangian/ } \\
\text { Karpatian }\end{array}$ & & leaves, fruits & \\
\hline & $\mathrm{HU}$ & Mecsek Mts. & 12 & $\begin{array}{l}\text { N } 46^{\circ} 14^{\prime} \\
\text { E } 18^{\circ} 17^{\prime}\end{array}$ & Hably (2020) & $\begin{array}{l}\text { late early } \\
\text { Miocene }\end{array}$ & $\begin{array}{l}\text { upper } \\
\text { Burdigalian }\end{array}$ & Karpatian & $\begin{array}{c}16.82 \pm 0.65 \\
\text { K/Ar date } \\
\text { from } \\
\text { fossiliferous } \\
\text { Komló } \\
\text { claymarl }\end{array}$ & leaves & \\
\hline & $\mathrm{DE}$ & Wackersdorf & 13 & $\begin{array}{l}\text { N 49 } 19^{\circ} 50.96 " \\
\text { E 12'10'39.46" }\end{array}$ & $\begin{array}{l}\text { Knobloch and } \\
\text { Kvaček (1976), } \\
\text { Gregor (1978), } \\
\text { Günther and } \\
\text { Gregor (1993) }\end{array}$ & $\begin{array}{l}\text { early } \\
\text { Miocene }\end{array}$ & Burdigalian & Karpatian & $14.5-17.3$ & leaves, fruits & \\
\hline & $C Z$ & Horní Bříza & 14 & $\begin{array}{l}\text { N 4950'28.79" } \\
\text { E } 13^{\circ} 21^{\prime} 28.56^{\prime \prime}\end{array}$ & $\begin{array}{l}\text { Němejc et al. } \\
(2003)\end{array}$ & $\begin{array}{l}\text { middle } \\
\text { Miocene }\end{array}$ & Serrevallian & $\begin{array}{l}\text { upper Badenian } \\
\text { / lower } \\
\text { Sarmatian }\end{array}$ & $11.6-13.8$ & leaves, fruits & \\
\hline & $\mathrm{DE}$ & Randeck Maar & 15 & $\begin{array}{l}\text { N 48 } 34^{\circ} 08^{\prime \prime} \\
\text { E } 9^{\circ} 30^{\prime} 77^{\prime \prime}\end{array}$ & $\begin{array}{l}\text { Rüffle (1963), } \\
\text { Rasser et al. (2013) }\end{array}$ & $\begin{array}{l}\text { late early/ } \\
\text { early middle } \\
\text { Miocene }\end{array}$ & $\begin{array}{l}\text { upper } \\
\text { Burdigalian/ } \\
\text { Langhian }\end{array}$ & $\begin{array}{l}\text { Neogene } \\
\text { Mammal zone } \\
\text { MN } 5\end{array}$ & & $\begin{array}{l}\text { leaves, fruits, } \\
\text { pollen }\end{array}$ & \\
\hline & AT & Parschlug & 16 & $\begin{array}{l}\text { N } 47^{\circ} 28^{\prime} 50.7^{\prime \prime} \\
\text { E } 15^{\circ} 17^{\prime} 15.3^{\prime \prime}\end{array}$ & $\begin{array}{l}\text { Kovar-Eder et al. } \\
(2004)\end{array}$ & $\begin{array}{l}\text { early/middle } \\
\text { Miocene }\end{array}$ & $\begin{array}{l}\text { uppermost } \\
\text { Burdigalian/ } \\
\text { lowermost } \\
\text { Langhian }\end{array}$ & $\begin{array}{l}\text { Karpatian/lower } \\
\text { Badenian }\end{array}$ & $?$ & leaves & \\
\hline
\end{tabular}


TABLE 1 (continued).

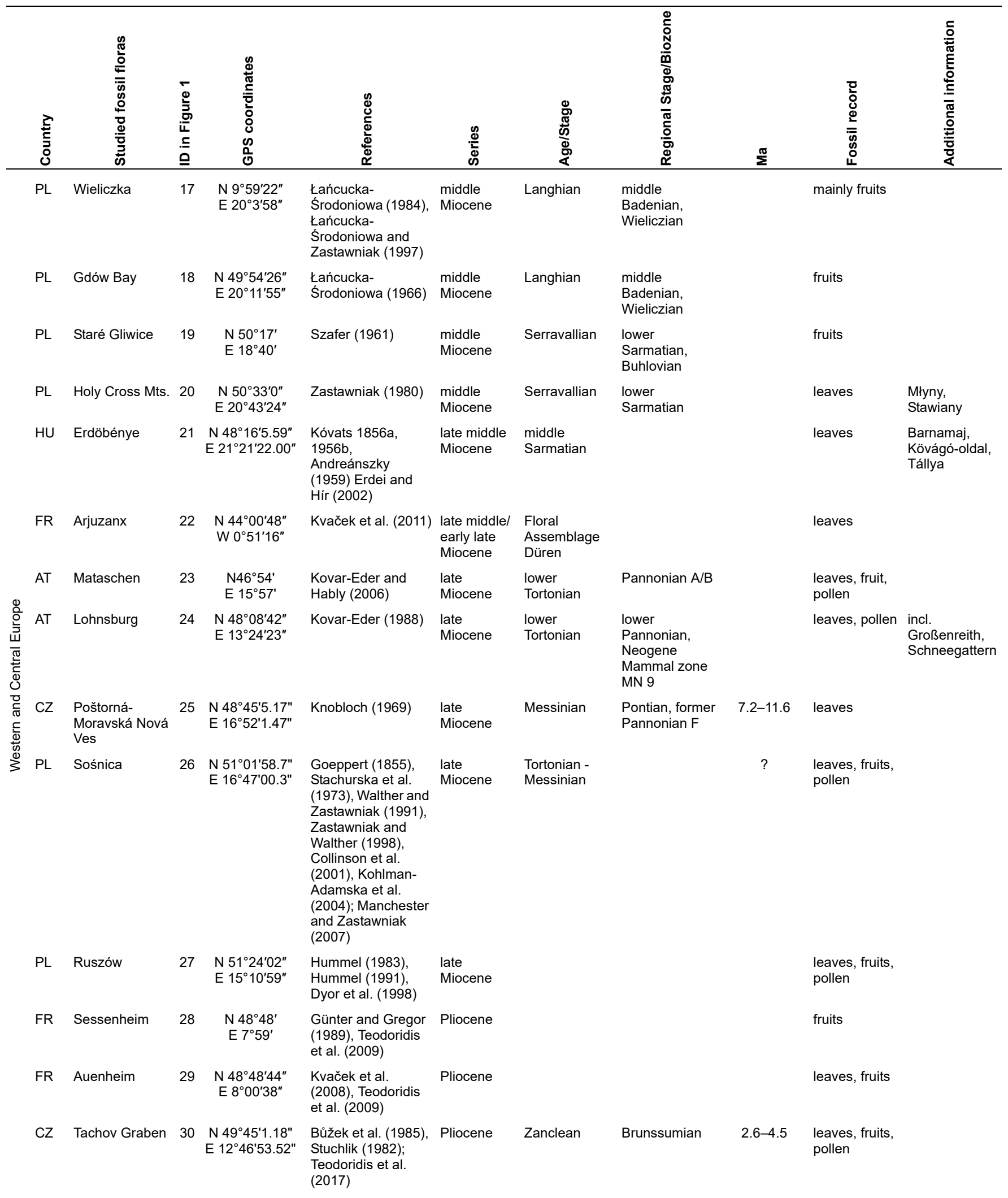


TABLE 1 (continued).

\begin{tabular}{|c|c|c|c|c|c|c|c|c|c|c|c|}
\hline & 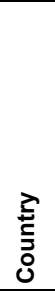 & 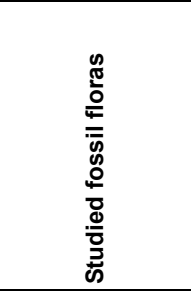 & 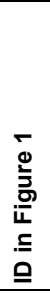 & 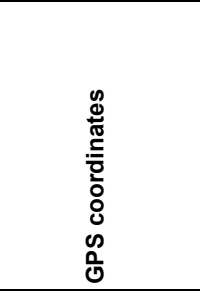 & 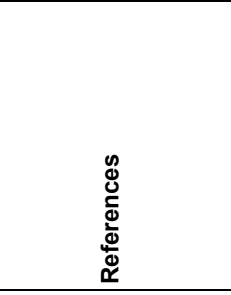 & \& & 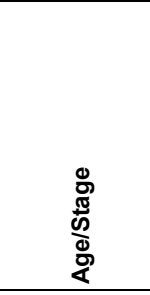 & 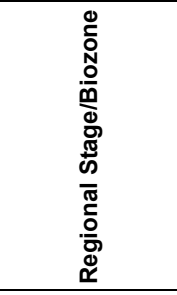 & $\sum^{\pi}$ & 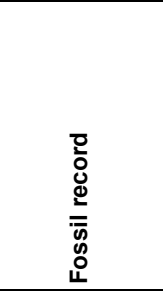 & 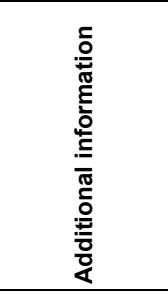 \\
\hline \multirow{11}{*}{ 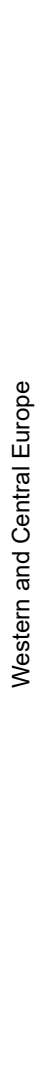 } & $\mathrm{DE}$ & Kaltensundheim & 31 & $\begin{array}{l}\text { N } 50^{\circ} 36^{\prime} 21.643^{\prime \prime} \\
\text { E } 10^{\circ} 09^{\prime} 22.740 "\end{array}$ & $\begin{array}{l}\text { Mai and Walther } \\
\text { (1988) }\end{array}$ & $\begin{array}{l}\text { late } \\
\text { Pliocene }\end{array}$ & Piacenzian & Reuverian & 3.4 & fruits & \\
\hline & $\mathrm{CZ}$ & $\begin{array}{l}\text { Vildštejn Fm. } \\
\text { Pluto Clay }\end{array}$ & 32 & $\begin{array}{l}\text { N } 50^{\circ} 09^{\prime} 19.74 " \\
\text { E } 12^{\circ} 22^{\prime} 43.99^{\prime \prime}\end{array}$ & $\begin{array}{l}\text { Bůžek et al. (1985), } \\
\text { Stuchlik (1982); } \\
\text { Teodoridis et al. } \\
\text { (2017) }\end{array}$ & $\begin{array}{l}\text { late } \\
\text { Pliocene }\end{array}$ & Piacenzian & Reuverian & $? 2.6-4.5$ & $\begin{array}{l}\text { leaves, fruits, } \\
\text { pollen }\end{array}$ & Vonšov Mb. \\
\hline & $\mathrm{CZ}$ & $\begin{array}{l}\text { Vildštejn Fm., } \\
\text { Nero Clay }\end{array}$ & 33 & $\begin{array}{l}\text { N 5009'19.74", } \\
\text { E } 12^{\circ} 22^{\prime} 43.99^{\prime \prime}\end{array}$ & $\begin{array}{l}\text { Bůžek et al. (1985), } \\
\text { Stuchlik (1982); } \\
\text { Teodoridis et al. } \\
\text { (2017) }\end{array}$ & Pliocene & Piacenzian & Reuverian & ? 2.6-4.5 & fruits, pollen & Nová Ves Mb. \\
\hline & $\mathrm{DE}$ & Gerstungen & 34 & $\begin{array}{c}\text { N } 50^{\circ} 57^{\prime} 49.799^{\prime \prime} \\
\text { E } 10^{\circ} 04^{\prime} 5.099^{\prime \prime}\end{array}$ & $\begin{array}{l}\text { Mai and Walther } \\
\text { (1988) }\end{array}$ & $\begin{array}{l}\text { Pliocene, ? } \\
\text { early, ? late }\end{array}$ & & & & fruits & \\
\hline & $\mathrm{DE}$ & Kranichfeld & 35 & $\begin{array}{l}\text { N } 50^{\circ} 51^{\prime} 25.596^{\prime \prime} \\
\text { E } 11^{\circ} 12^{\prime} 11.499^{\prime \prime}\end{array}$ & $\begin{array}{l}\text { Mai and Walther } \\
\text { (1988) }\end{array}$ & $\begin{array}{l}\text { late } \\
\text { Pliocene }\end{array}$ & Piacenzian & Reuverian & & fruits & \\
\hline & $\mathrm{DE}$ & Berga & 36 & $\begin{array}{l}\text { N } 51^{\circ} 27^{\prime} 21.355^{\prime \prime} \\
\text { E } 11^{\circ} 0 ' 30.913^{\prime \prime}\end{array}$ & $\begin{array}{l}\text { Mai and Walther } \\
\text { (1988) }\end{array}$ & $\begin{array}{l}\text { late } \\
\text { Pliocene }\end{array}$ & Piacenzian & Reuverian & 2.5 & leaves, fruits & \\
\hline & $\mathrm{CZ}$ & $\begin{array}{l}\text { Vildštejn Fm., } \\
\text { lignite beds }\end{array}$ & 37 & $\begin{array}{c}\text { N } 50^{\circ} 09^{\prime} 19.74 " \\
\text { E } 12^{\circ} 22^{\prime} 43.99^{\prime \prime}\end{array}$ & $\begin{array}{l}\text { Bůžek et al. (1985), } \\
\text { Stuchlik (1982); } \\
\text { Teodoridis et al. } \\
\text { (2017) }\end{array}$ & Pleistocene & Piacenzian & Reuverian & $1.5-2.6$ & $\begin{array}{l}\text { leaves, fruits, } \\
\text { pollen }\end{array}$ & Nová Ves Mb. \\
\hline & $\mathrm{DE}$ & Rippersroda & 38 & $\begin{array}{l}\text { N } 50^{\circ} 46^{\prime} 14.94 " \\
\text { E } 50^{\circ} 38^{\prime} 29.13^{\prime \prime}\end{array}$ & $\begin{array}{l}\text { Mai and Walther } \\
\text { (1988) }\end{array}$ & $\begin{array}{l}\text { late } \\
\text { Pliocene/ } \\
\text { Pleistocene }\end{array}$ & Reuverian & Tiglian C & $1.5-2.6$ & fruits & \\
\hline & $C Z$ & $\begin{array}{l}\text { Vildštejn Fm., } \\
\text { upper beds }\end{array}$ & 39 & $\begin{array}{l}\text { N } 50^{\circ} 09^{\prime} 19.74 " \\
\text { E } 12^{\circ} 22^{\prime} 43.99^{\prime \prime}\end{array}$ & $\begin{array}{l}\text { Bůžek et al. (1985), } \\
\text { Stuchlik (1982); } \\
\text { Teodoridis et al. } \\
\text { (2017) }\end{array}$ & Pleistocene & & Praetiglian & $1.5-2.6$ & $\begin{array}{l}\text { (leaves, } \\
\text { fruits), pollen }\end{array}$ & Nová Ves Mb. \\
\hline & $\mathrm{DE}$ & Nordhausen & 40 & $\begin{array}{l}\text { N } 51^{\circ} 29^{\prime} 51.47^{\prime \prime} \\
\text { E } 10^{\circ} 47^{\prime} 50.59^{\prime \prime}\end{array}$ & $\begin{array}{l}\text { Mai and Walther } \\
\text { (1988) }\end{array}$ & $\begin{array}{l}\text { latest } \\
\text { Pliocene/ } \\
\text { Pleistocene }\end{array}$ & $\begin{array}{l}\text { uppermost } \\
\text { Pliocene to } \\
\text { Tiglian A }\end{array}$ & & 1.65 & fruits & \\
\hline & $\mathrm{DE}$ & Klinger beds & 41 & $\begin{array}{l}\text { N } 51^{\circ} 45^{\prime} 38^{\prime \prime} \\
\text { E } 14^{\circ} 20^{\prime} 03^{\prime \prime}\end{array}$ & Striegler (2008) & Pleistocene & $\begin{array}{l}\text { Eem } \\
\text { Interglacial }\end{array}$ & & & leaves & \\
\hline \multirow{7}{*}{ 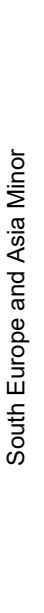 } & TY & Güvem & 42 & $\begin{array}{l}\text { N } 40^{\circ} 28^{\prime} 11^{\prime \prime} \\
\text { E } 32^{\circ} 39^{\prime} 02^{\prime \prime}\end{array}$ & Denk et al. (2017) & $\begin{array}{l}\text { early } \\
\text { Miocene }\end{array}$ & Burdigalian & $\begin{array}{l}\text { Neogene } \\
\text { Mammal Zone } \\
\text { MN3 }\end{array}$ & $20-18$ & leaves & \\
\hline & GR & Kymi & 43 & $\begin{array}{l}\text { N } 38^{\circ} 38^{\prime} \\
\text { E } 24^{\circ} 06^{\prime}\end{array}$ & $\begin{array}{l}\text { Velitzelos et al. } \\
(2002)\end{array}$ & $\begin{array}{l}\text { early } \\
\text { Miocene }\end{array}$ & & & 13 & leaves & \\
\hline & TY & Yatagan Basin & 44 & $\begin{array}{l}\text { N } 37^{\circ} 35^{\prime} \\
\text { E } 28^{\circ} 14^{\prime}\end{array}$ & Güner et al. (2017) & $\begin{array}{l}\text { middle } \\
\text { Miocene }\end{array}$ & $\begin{array}{l}\text { upper } \\
\text { Langhian/ } \\
\text { lower } \\
\text { Serravallian }\end{array}$ & & & leaves & \\
\hline & GR & Pitsidia & 45 & $\begin{array}{c}\text { N } 34^{\circ} 59^{\prime} 43.99^{\prime \prime} \\
\text { E } 24^{\circ} 45^{\prime} 0.03^{\prime \prime}\end{array}$ & $\begin{array}{l}\text { Zidianakis et al. } \\
2015,2016,2020)\end{array}$ & $\begin{array}{l}\text { late } \\
\text { Miocene }\end{array}$ & $\begin{array}{l}\text { lower-upper } \\
\text { Tortonian }\end{array}$ & & & leaves & \\
\hline & IT & Mt. Tondo & 46 & $\begin{array}{l}\mathrm{N} 44^{\circ} 17^{\prime} \\
\mathrm{E} 11^{\circ} 35^{\prime}\end{array}$ & $\begin{array}{l}\text { Teodoridis et al. } \\
(2015)\end{array}$ & $\begin{array}{l}\text { late } \\
\text { Miocene }\end{array}$ & Messinian & & appr. 5.7 & mainly leaves & $\begin{array}{l}\text { Vena del } \\
\text { Gesso Fm. }\end{array}$ \\
\hline & IT & Tossignano & 47 & 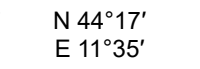 & $\begin{array}{l}\text { Teodoridis et al. } \\
(2015)\end{array}$ & $\begin{array}{l}\text { late } \\
\text { Miocene }\end{array}$ & Messinian & & appr. 5.7 & leaves, pollen & $\begin{array}{l}\text { Vena del } \\
\text { Gesso Fm. }\end{array}$ \\
\hline & IT & Ca'Viettone & 48 & $\begin{array}{l}\mathrm{N} 45^{\circ} 19^{\prime} \\
\mathrm{E} 7^{\circ} 36^{\prime}\end{array}$ & $\begin{array}{l}\text { Bertoldi and } \\
\text { Martinetto (1995) }\end{array}$ & Pliocene & $\begin{array}{l}\text { upper } \\
\text { Zanclean } \\
\text { (probably) }\end{array}$ & & & $\begin{array}{l}\text { leaves, } \\
\text { pollen, fruits }\end{array}$ & \\
\hline
\end{tabular}


TABLE 1 (continued).

\begin{tabular}{|c|c|c|c|c|c|c|c|c|c|c|c|}
\hline & 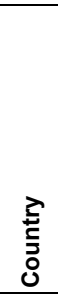 & 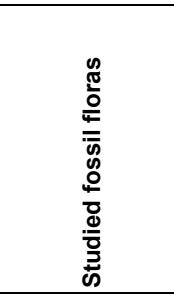 & 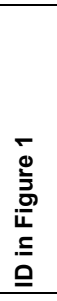 & 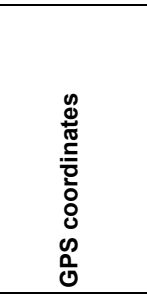 & 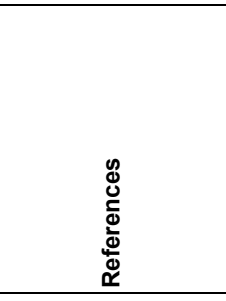 & 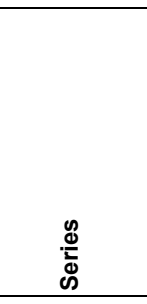 & 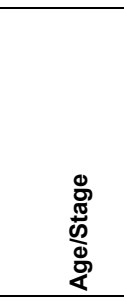 & 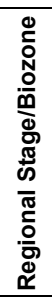 & $\sum^{\pi}$ & 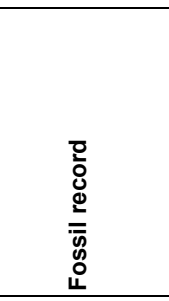 & 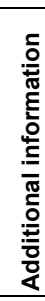 \\
\hline \multirow{6}{*}{ 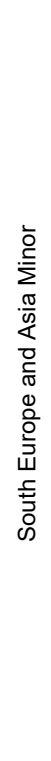 } & AM & Shamb $1 b+A 1$ & 49 & $\begin{array}{l}\text { N } 39^{\circ} 28^{\prime} 03^{\prime \prime} \\
\text { E } 46^{\circ} 09^{\prime} 00^{\prime \prime}\end{array}$ & $\begin{array}{l}\text { Bruch and } \\
\text { Gabrielyan (2002), } \\
\text { Bruch et al. } \\
\text { (unpublished) }\end{array}$ & $\begin{array}{l}\text { early } \\
\text { Pleistocene }\end{array}$ & Calabrian & & 1.298 & leaves, pollen & \\
\hline & AM & Shamb 1f+B1 & 50 & $\begin{array}{l}\text { N } 39^{\circ} 28^{\prime} 03^{\prime \prime} \\
\text { E } 46^{\circ} 09^{\prime} 00^{\prime \prime}\end{array}$ & $\begin{array}{l}\text { Bruch and } \\
\text { Gabrielyan (2002), } \\
\text { Bruch et al. } \\
\text { (unpublished) }\end{array}$ & $\begin{array}{l}\text { early } \\
\text { Pleistocene }\end{array}$ & Calabrian & & 1.275 & leaves, pollen & \\
\hline & AM & Darbas $2 / d$ & 51 & $\begin{array}{l}\text { N } 39^{\circ} 26^{\prime} 24^{\prime \prime} \\
\text { E } 46^{\circ} 07^{\prime \prime} 20^{\prime \prime}\end{array}$ & $\begin{array}{l}\text { Bruch and } \\
\text { Gabrielyan (2002), } \\
\text { Bruch et al. } \\
\text { (unpublished) }\end{array}$ & $\begin{array}{l}\text { early } \\
\text { Pleistocene }\end{array}$ & Calabrian & & 1.0715 & leaves, pollen & \\
\hline & AM & $\begin{array}{l}\text { Uyts-2/a + } \\
\text { Uts-2 PZ23 }\end{array}$ & 52 & $\begin{array}{l}\text { N } 39^{\circ} 31^{\prime} \\
\text { E } 46^{\circ} 03^{\prime}\end{array}$ & $\begin{array}{l}\text { Bruch and } \\
\text { Gabrielyan (2002), } \\
\text { Bruch et al. } \\
\text { (unpublished) }\end{array}$ & $\begin{array}{l}\text { early } \\
\text { Pleistocene }\end{array}$ & Calabrian & & 1.0715 & leaves, pollen & \\
\hline & AM & $\begin{array}{l}\text { Darbas-2/f + } \\
\text { Drb-2 PZ6 }\end{array}$ & 53 & $\begin{array}{l}\text { N } 39^{\circ} 26^{\prime} 24^{\prime \prime} \\
\text { E } 46^{\circ} 07^{\prime \prime} 20^{\prime \prime}\end{array}$ & $\begin{array}{l}\text { Bruch and } \\
\text { Gabrielyan (2002), } \\
\text { Bruch et al. } \\
\text { (unpublished) }\end{array}$ & $\begin{array}{l}\text { early } \\
\text { Pleistocene }\end{array}$ & Calabrian & & 1.030 & leaves, pollen & \\
\hline & AM & Tolors 2 & 54 & $\begin{array}{l}\text { N } 39^{\circ} 27^{\prime} 38^{\prime \prime} \\
\text { E } 46^{\circ} 02^{\prime} 46^{\prime \prime}\end{array}$ & $\begin{array}{l}\text { Bruch and } \\
\text { Gabrielyan (2002), } \\
\text { Bruch et al. } \\
\text { (unpublished) }\end{array}$ & $\begin{array}{l}\text { early } \\
\text { Pleistocene }\end{array}$ & Calabrian & & 0.998 & leaves, pollen & \\
\hline
\end{tabular}

composition between a given fossil assemblage and modern vegetation units.

Drudges 1 and 2 display the closeness of the fossil and modern plant assemblages based on their proportions of key zonal components and the parameter Taxonomic Similarity (TS) based on cluster analysis (Ward's method, Euclidian distance). Drudge 1 is designed to extract the highest level of correspondence of the proportions of zonal woody angiosperms in the fossil assemblage and the taxon correspondence at the generic level (TS). Drudge 2 was developed to better distinguish more open vegetation types from closed ones. It works on the same principle as Drudge 1 but includes the zonal herb components (DRY HERB and MESO HERB). It determines the highest level of correspondence in the proportions of the zonal woody key components (BLD, BLE, SCL+LEG) of all zonal components (CONIFER, BLD, BLE, SCL+LEG, ZONAL PALM, ARBFERN, DRY HERB, and MESO HERB).

Since the publication of Teodoridis et al. (2020) some minor corrections have been made and the most recent versions of these tools (Drudge_1_505_corr_20200510, Drudge_2_505_corr_20200510) are provided here in Appendices 2 and 3. Furthermore, an online application is offered by Teodoridis et al. (2021). The results in this study are modern vegetation proxies for every single fossil plant assemblage by applying Drudges 1 and 2 (Table 2). They list the five best fitted results of the IPR Similarity, the Taxonomic Similarity (TS), and the Results Mix (hereafter "similarity approaches"). These results were plotted on a spreadsheet which constitutes the matrix of all the graphs presented here (Appendix $4)$. The fossil assemblages (x-axis) are plotted against the dataset of modern vegetation units ( $y$ axis). The different working sheets yield the results of the individual similarity approaches (IPR Similarities, TS, Results Mix based on Drudge 1 and 2) and the summary of these results (hereafter "overall scores") in the worknig sheet "Overall scores Drudge 1+2". The number of all proxies for all 54 assemblages in every similarity approach (IPR Similarity, TS, Results Mix of both Drudges) is 270 $(54 \times 5)$. Note that TS is counted only once because it is identical in Drudges 1 and 2. In the overall score all proxies from the different similarity approaches are summarised, i.e., for every site 25 proxies (five from each similarity approach). Hence, the number of all proxies for all sites and all 
TABLE 2. Results of applying the Drudges 1 and 2 on the test set of fossil assemblages. Listed are the five best fitted results for every site/assemblage for the IPR Similarity, Taxonomic Similarity (TS), and Results Mix (hereafter "similarity approaches") for both Drudges. For every individual site, 25 proxies are listed (five for each of the similarity approaches). Note that TS is counted only once because it is identical for Drudge 1 and 2 . The number for all proxies for every similarity approach is $270(54 \times 5)$. The number of all proxies for all sites and all similarity approaches is 1350 $(54 \times 5 \times 5)$.

\begin{tabular}{|c|c|c|c|c|c|c|c|c|c|c|c|c|c|}
\hline & & 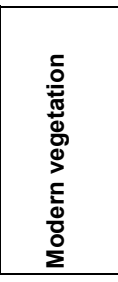 & 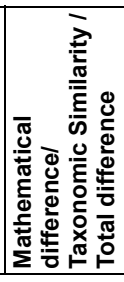 & 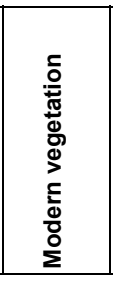 & 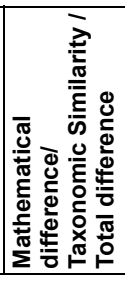 & 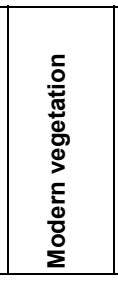 & 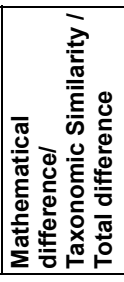 & 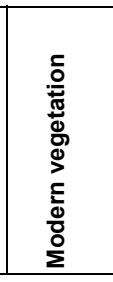 & 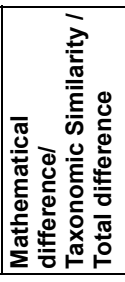 & 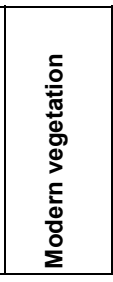 & 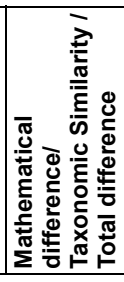 & 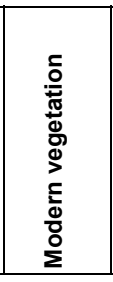 & 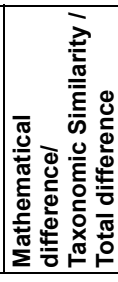 \\
\hline \multicolumn{14}{|c|}{ Drudge 1} \\
\hline Site/results & & \multicolumn{2}{|c|}{ Hampshire Basin } & \multicolumn{2}{|c|}{ London Clay } & \multicolumn{2}{|c|}{ Messel } & \multicolumn{2}{|c|}{ Geiseltal } & \multicolumn{2}{|c|}{ Profen-Scheiplitz } & \multicolumn{2}{|c|}{ Staré Sedlo } \\
\hline \multirow{5}{*}{$\begin{array}{l}\text { Results - IPR } \\
\text { Similarity }\end{array}$} & 1 & China 17 & 3.0 & China 17 & 3.2 & Japan 07 & 2.1 & Japan 07 & 6.2 & Japan 13 & 4.0 & Japan 08 & 4.0 \\
\hline & 2 & China 89 & 4.3 & China 34 & 4.4 & Japan 08 & 6.6 & China 18 & 7.3 & Japan 12 & 4.1 & D032 & 5.3 \\
\hline & 3 & D032 & 4.7 & China 32 & 4.5 & China 34 & 6.8 & China 34 & 9.6 & China 16 & 4.5 & China 89 & 5.4 \\
\hline & 4 & China 32 & 4.9 & D032 & 5.8 & D032 & 6.9 & China 19 & 11.0 & Japan 11 & 4.8 & Japan 07 & 7.2 \\
\hline & 5 & China 34 & 6.5 & Japan 07 & 6.7 & China 17 & 8.6 & Japan 08 & 11.4 & China 22 & 5.7 & China 17 & 8.0 \\
\hline \multirow{5}{*}{$\begin{array}{l}\text { Results - } \\
\text { Taxonomic } \\
\text { Similarity }\end{array}$} & 1 & Japan 06 & $18.3 \%$ & Japan 06 & $13.5 \%$ & China 17 & $7.5 \%$ & China 57 & $17.9 \%$ & China 17 & $23.7 \%$ & China 34 & $5.7 \%$ \\
\hline & 2 & China 77 & $18.3 \%$ & China 57 & $12.7 \%$ & Japan 14 & $6.8 \%$ & China 61 & $14.9 \%$ & China 21 & $21.1 \%$ & China 38 & $5.0 \%$ \\
\hline & 3 & Japan 01 & $17.2 \%$ & China 77 & $12.7 \%$ & China 57 & $6.2 \%$ & China 38 & $13.4 \%$ & Japan 11 & $21.1 \%$ & China 13 & $5.0 \%$ \\
\hline & 4 & Japan 02 & $17.2 \%$ & Japan 01 & $12.7 \%$ & Japan 01 & $6.2 \%$ & China 58 & $13.4 \%$ & Japan 12 & $21.1 \%$ & China 14 & $5.0 \%$ \\
\hline & 5 & China 57 & $17.2 \%$ & Japan 02 & $12.7 \%$ & Japan 02 & $6.2 \%$ & China 60 & $11.9 \%$ & China 38 & $21.1 \%$ & Japan 01 & $5.0 \%$ \\
\hline \multirow{5}{*}{ Results - Mix } & 1 & China 17 & 85.0 & China 17 & 89.7 & China 17 & 92.9 & China 34 & 90.1 & Japan 12 & 79.1 & China 34 & 94.8 \\
\hline & 2 & \begin{tabular}{|l|} 
China 32 \\
\end{tabular} & 88.3 & na 32 & 91.4 & 08 & 95.4 & China 32 & 90.5 & Japan 11 & 79.1 & China 36 & 96.9 \\
\hline & 3 & China 01 & 89.8 & China 01 & 91.6 & Japan 14 & 96.0 & China 33 & 91.8 & China 21 & 79.8 & China 89 & 97.3 \\
\hline & 4 & China 36 & 90.9 & China 36 & 93.1 & China 19 & 96.8 & China 17 & 91.9 & Japan 10 & 81.8 & China 30 & 97.6 \\
\hline & 5 & China 33 & 91.0 & China 33 & 93.3 & China 33 & 96.9 & China 36 & 93.4 & China 20 & 81.9 & China 32 & 97.7 \\
\hline \multicolumn{14}{|c|}{ Drudge 2} \\
\hline Site/results & & \multicolumn{2}{|c|}{ Hampshire Basin } & \multicolumn{2}{|c|}{ London Clay } & \multicolumn{2}{|c|}{ Messel } & \multicolumn{2}{|c|}{ Geiseltal } & \multicolumn{2}{|c|}{ Profen-Scheiplitz } & \multicolumn{2}{|c|}{ Staré Sedlo } \\
\hline \multirow{5}{*}{$\begin{array}{l}\text { Results - IPR } \\
\text { Similarity }\end{array}$} & 1 & \begin{tabular}{|l|} 
China 32 \\
\end{tabular} & 4.8 & China 34 & 3.3 & China 34 & 8.6 & China 30 & 7.8 & China 20 & 10.3 & China 89 & 12.8 \\
\hline & 2 & China 34 & 6.4 & China 32 & 4.3 & China 19 & 11.4 & China 19 & 13.3 & China 16 & 12.9 & China 30 & 12.8 \\
\hline & 3 & China 36 & 10.5 & China 33 & 12.1 & China 32 & 12.4 & China 33 & 13.5 & China 21 & 14.1 & China 36 & 17.4 \\
\hline & 4 & China 01 & 12.7 & China 01 & 12.2 & China 33 & 12.4 & China 34 & 14.8 & China 22 & 15.0 & China 17 & 17.5 \\
\hline & 5 & China 19 & 15.9 & China 19 & 12.7 & China 30 & 13.5 & China 21 & 16.1 & China 31 & 15.0 & China 34 & 17.5 \\
\hline \multirow{5}{*}{$\begin{array}{l}\text { Results - } \\
\text { Taxonomic } \\
\text { Similarity }\end{array}$} & 1 & Japan 06 & $18.3 \%$ & Japan 06 & $13.5 \%$ & China 17 & $7.5 \%$ & China 57 & $17.9 \%$ & China 17 & $23.7 \%$ & China 34 & $5.7 \%$ \\
\hline & 2 & \begin{tabular}{|l|} 
China 77 \\
\end{tabular} & $18.3 \%$ & China 57 & $12.7 \%$ & \begin{tabular}{|l|} 
Japan 14 \\
\end{tabular} & $6.8 \%$ & China 61 & $14.9 \%$ & China 21 & $21.1 \%$ & China 38 & $5.0 \%$ \\
\hline & 3 & \begin{tabular}{|l|} 
Japan 01 \\
\end{tabular} & $17.2 \%$ & China 77 & $12.7 \%$ & China 57 & $6.2 \%$ & China 38 & $13.4 \%$ & Japan 11 & $21.1 \%$ & China 13 & $5.0 \%$ \\
\hline & 4 & Japan 02 & $17.2 \%$ & Japan 01 & $12.7 \%$ & Japan 01 & $6.2 \%$ & China 58 & $13.4 \%$ & |Japan 12 & $21.1 \%$ & China 14 & $5.0 \%$ \\
\hline & 5 & China 57 & $17.2 \%$ & Japan 02 & $12.7 \%$ & Japan 02 & $6.2 \%$ & China 60 & $11.9 \%$ & China 38 & $21.1 \%$ & Japan 01 & $5.0 \%$ \\
\hline \multirow{5}{*}{ Results - Mix } & 1 & China 32 & 88.3 & China 32 & 91.4 & China 17 & 95.7 & China 34 & 90.8 & China 21 & 80.2 & China 34 & 95.9 \\
\hline & 2 & China 01 & 90.2 & China 01 & 92.1 & China 19 & 96.6 & China 32 & 91.5 & Japan 12 & 82.1 & China 30 & 96.6 \\
\hline & 3 & China 17 & 90.3 & China 33 & 92.9 & China 33 & 96.7 & China 33 & 92.0 & Japan 11 & 82.2 & China 38 & 97.7 \\
\hline & 4 & China 33 & 90.7 & China 36 & 93.0 & China 21 & 97.6 & China 38 & 92.3 & China 20 & 82.2 & China 36 & 98.0 \\
\hline & 5 & China 36 & 90.9 & China 17 & 94.4 & China 34 & 97.6 & China 17 & 93.3 & China 31 & 82.9 & China 89 & 98.0 \\
\hline
\end{tabular}


TABLE 2 (continued).

\begin{tabular}{|c|c|c|c|c|c|c|c|c|c|c|c|c|c|}
\hline & & 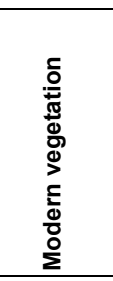 & 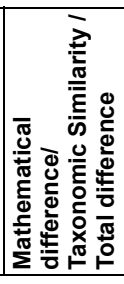 & 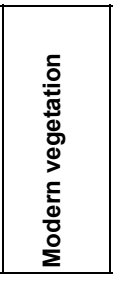 & 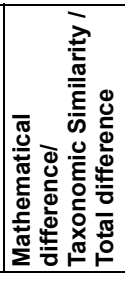 & 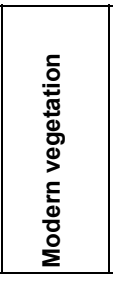 & 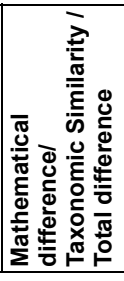 & 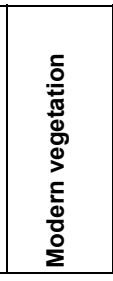 & 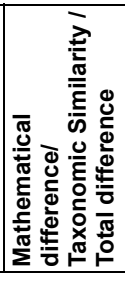 & 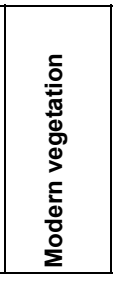 & 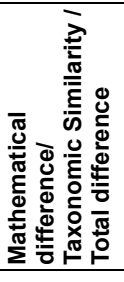 & 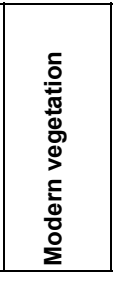 & 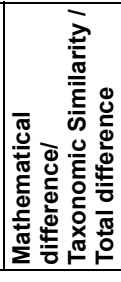 \\
\hline \multicolumn{14}{|c|}{ Drudge 1} \\
\hline Site/results & & \multicolumn{2}{|c|}{ Flörsheim } & \multicolumn{2}{|c|}{ Rauenberg } & \multicolumn{2}{|c|}{ Seifhennersdorf } & \multicolumn{2}{|c|}{$\begin{array}{c}\text { Linz, Ebelsberg } \\
\text { Fm. }\end{array}$} & \multicolumn{2}{|c|}{ Oberdorf } & \multicolumn{2}{|c|}{ Mecsek Mts. } \\
\hline \multirow{5}{*}{$\begin{array}{l}\text { Results - IPR } \\
\text { Similarity }\end{array}$} & 1 & China 18 & 6.4 & J037 & 14.5 & China 84 & 4.9 & H001 & 1.6 & H001 & 1.4 & F001 & 1.7 \\
\hline & 2 & Japan 07 & 9.5 & Japan 07 & 15.0 & F166 & 4.9 & C045 & 5.4 & China 36 & 4.6 & C008 & 2.8 \\
\hline & 3 & China 19 & 10.3 & China 18 & 15.7 & F169 & 5.1 & Japan 04 & 5.5 & C045 & 6.0 & F030 & 3.2 \\
\hline & 4 & China 33 & 10.7 & |Japan 08 & 18.7 & China 38 & 5.2 & China 35 & 5.9 & |Japan $04 \mid$ & 7.1 & D056 & 4.4 \\
\hline & 5 & China 34 & 11.9 & China 19 & 19.4 & China 28 & 7.2 & China 37 & 5.9 & China 35 & 7.5 & D042 & 4.9 \\
\hline \multirow{5}{*}{$\begin{array}{l}\text { Results - } \\
\text { Taxonomic } \\
\text { Similarity }\end{array}$} & 1 & Japan 05 & $15.9 \%$ & China 35 & $22.8 \%$ & Japan 05 & $41.2 \%$ & China 62 & $38.7 \%$ & China 60 & $31.5 \%$ & China 78 & $27.6 \%$ \\
\hline & 2 & China 38 & $15.9 \%$ & China 37 & $22.8 \%$ & China 57 & $41.2 \%$ & Japan 01 & $35.5 \%$ & Japan 05 & $30.0 \%$ & Japan 05 & $26.3 \%$ \\
\hline & 3 & China 77 & $14.3 \%$ & China 34 & $19.3 \%$ & China 67 & $41.2 \%$ & China 60 & $35.5 \%$ & |Japan $06 \mid$ & $26.2 \%$ & |China 67 & $26.3 \%$ \\
\hline & 4 & J019 & $14.3 \%$ & China 38 & $17.5 \%$ & China 60 & $39.7 \%$ & China 67 & $32.3 \%$ & Japan 14 & $25.4 \%$ & China 61 & $25.0 \%$ \\
\hline & 5 & J022 & $14.3 \%$ & F009 & $17.5 \%$ & China 61 & $39.7 \%$ & China 57 & $32.3 \%$ & China 57 & $25.4 \%$ & China 68 & $23.7 \%$ \\
\hline \multirow{5}{*}{ Results - Mix } & 1 & China 34 & 88.1 & China 34 & 83.1 & China 67 & 60.3 & China 67 & 72.7 & China 17 & 76.6 & $\mathrm{H} 002$ & 83.9 \\
\hline & 2 & China 33 & 89.5 & China 33 & 84.8 & China 56 & 65.0 & China 32 & 74.0 & China 36 & 82.4 & China 67 & 83.9 \\
\hline & 3 & Japan 11 & 89.8 & China 35 & 86.4 & China 57 & 67.1 & China 36 & 74.5 & China 67 & 82.6 & F156 & 85.7 \\
\hline & 4 & Japan 08 & 90.1 & China 37 & 86.4 & China 60 & 67.9 & China 62 & 75.5 & Japan 04 & 82.6 & J017 & 85.8 \\
\hline & 5 & Japan 12 & 90.2 & China 22 & 88.3 & China 61 & 68.8 & China 59 & 78.4 & China 60 & 83.0 & China 14 & 86.3 \\
\hline \multicolumn{14}{|c|}{ Drudge 2} \\
\hline Site/results & & \multicolumn{2}{|c|}{ Flörsheim } & \multicolumn{2}{|c|}{ Rauenberg } & \multicolumn{2}{|c|}{ Seifhennersdorf } & \multicolumn{2}{|c|}{$\begin{array}{l}\text { Linz, Ebelsberg } \\
\text { Fm. }\end{array}$} & \multicolumn{2}{|c|}{ Oberdorf } & \multicolumn{2}{|c|}{ Mecsek Mts. } \\
\hline \multirow{5}{*}{$\begin{array}{l}\text { Results - IPR } \\
\text { Similarity }\end{array}$} & 1 & China 33 & 10.3 & China 33 & 16.1 & China 38 & 4.9 & China 38 & 15.9 & Japan 04 & 10.7 & D042 & 16.2 \\
\hline & 2 & China 19 & 13.0 & China 30 & 18.5 & China 59 & 9.7 & China 89 & 16.5 & China 36 & 11.5 & D002 & 20.6 \\
\hline & 3 & China 30 & 13.1 & China 19 & 19.4 & China 67 & 10.0 & China 36 & 19.0 & China 38 & 11.8 & G076 & 23.4 \\
\hline & 4 & China 34 & 13.7 & China 34 & 19.7 & Japan 04 & 10.5 & China 59 & 20.3 & China 35 & 13.7 & D045 & 24.1 \\
\hline & 5 & China 21 & 17.0 & Japan 09 & 21.5 & China 35 & 12.9 & Japan 04 & 20.4 & China 37 & 13.7 & D064 & 24.3 \\
\hline \multirow{5}{*}{$\begin{array}{l}\text { Results - } \\
\text { Taxonomic } \\
\text { Similarity }\end{array}$} & 1 & Japan 05 & $16 \%$ & China 35 & $23 \%$ & Japan 05 & $41.2 \%$ & China 62 & $39 \%$ & China 60 & $31.5 \%$ & China 78 & $27.6 \%$ \\
\hline & 2 & China 38 & $16 \%$ & China 37 & $23 \%$ & China 57 & $41.2 \%$ & Japan 01 & $36 \%$ & Japan 05 & $30.0 \%$ & Japan 05 & $26.3 \%$ \\
\hline & 3 & China 77 & $14 \%$ & China 34 & $19 \%$ & China 67 & $41.2 \%$ & China 60 & $36 \%$ & Japan 06 & $26.2 \%$ & China 67 & $26.3 \%$ \\
\hline & 4 & J019 & $14 \%$ & China 38 & $18 \%$ & China 60 & $39.7 \%$ & China 67 & $32 \%$ & Japan 14 & $25.4 \%$ & China 61 & $25.0 \%$ \\
\hline & 5 & J022 & $14 \%$ & F009 & $18 \%$ & China 61 & $39.7 \%$ & China 57 & $32 \%$ & China 57 & $25.4 \%$ & China 68 & $23.7 \%$ \\
\hline \multirow{5}{*}{ Results - Mix } & 1 & China 34 & 88.4 & China 34 & 83.1 & China 67 & 59.7 & China 67 & 72.7 & China 17 & 78.4 & China 67 & 81.1 \\
\hline & 2 & China 33 & 89.5 & China 33 & 84.0 & China $57 \mid$ & 65.0 & China 62 & 72.9 & China 60 & 80.4 & J017 & 85.9 \\
\hline & 3 & China 36 & 90.3 & China 35 & 85.4 & China 56 & 65.3 & China 32 & 76.4 & China 67 & 81.7 & China 59 & 86.6 \\
\hline & 4 & Japan 11 & 90.4 & China 37 & 85.4 & China 60 & 66.0 & China 36 & 76.6 & China 33 & 82.8 & G056 & 87.9 \\
\hline & 5 & Japan 12 & 90.5 & China 30 & 87.9 & China 58 & 68.0 & China 59 & 76.9 & |Japan 04 & 83.0 & D052 & 89.3 \\
\hline
\end{tabular}


TABLE 2 (continued).

\begin{tabular}{|c|c|c|c|c|c|c|c|c|c|c|c|c|c|}
\hline & & 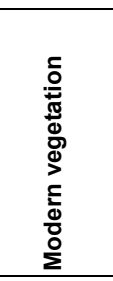 & 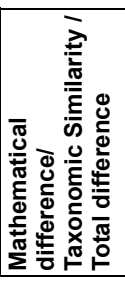 & 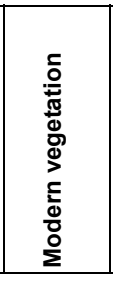 & 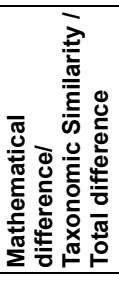 & 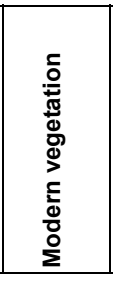 & 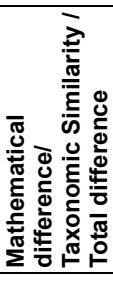 & 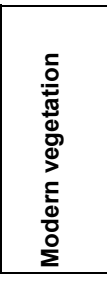 & 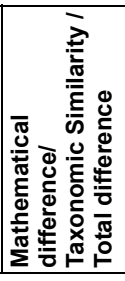 & 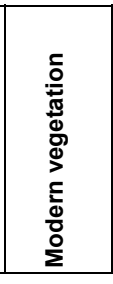 & 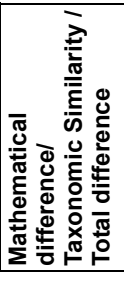 & 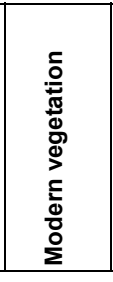 & 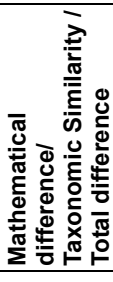 \\
\hline \multicolumn{14}{|c|}{ Drudge 1} \\
\hline Site/results & & \multicolumn{2}{|c|}{ Wackersdorf } & \multicolumn{2}{|c|}{ Horní Bříza } & \multicolumn{2}{|c|}{ Randeck Maar } & \multicolumn{2}{|c|}{ Parschlug } & \multicolumn{2}{|c|}{ Wieliczka } & \multicolumn{2}{|c|}{ Gdów Bay } \\
\hline \multirow{5}{*}{$\begin{array}{l}\text { Results - IPR } \\
\text { Similarity }\end{array}$} & 1 & Japan 04 & 2.2 & F139 & 3.2 & F026 & 3.1 & G043 & 2.2 & F169 & 1.8 & F107 & 2.5 \\
\hline & 2 & C045 & 2.6 & F137 & 3.3 & D030 & 4.2 & F098 & 2.6 & Japan 15 & 4.5 & F117 & 3.6 \\
\hline & 3 & F163 & 3.6 & China 13 & 3.9 & D011 & 4.7 & K022 & 2.7 & China 38 & 5.1 & Japan 02 & 3.7 \\
\hline & 4 & H001 & 4.0 & D064 & 4.1 & D056 & 5.0 & G074 & 3.4 & China 84 & 5.7 & China 79 & 3.8 \\
\hline & 5 & China 35 & 5.1 & F084 & 5.0 & K009 & 5.4 & K015 & 4.0 & C042 & 6.5 & F157 & 4.2 \\
\hline \multirow{5}{*}{$\begin{array}{c}\text { Results - } \\
\text { Taxonomic } \\
\text { Similarity }\end{array}$} & 1 & Japan 06 & $31.0 \%$ & China 57 & $46.4 \%$ & China 57 & $23.8 \%$ & China 68 & $32.8 \%$ & Japan 06 & $39.2 \%$ & Japan 05 & $37.5 \%$ \\
\hline & 2 & Japan 05 & $29.1 \%$ & China 67 & $42.9 \%$ & Japan 05 & $21.4 \%$ & China 81 & $31.3 \%$ & Japan 05 & $37.7 \%$ & China 60 & $32.5 \%$ \\
\hline & 3 & China 57 & $27.8 \%$ & China 68 & $39.3 \%$ & China 56 & $21.4 \%$ & Japan 05 & $29.7 \%$ & China 57 & $36.2 \%$ & China 68 & $30.0 \%$ \\
\hline & 4 & China 60 & $27.8 \%$ & China 78 & $39.3 \%$ & China 68 & $20.2 \%$ & Japan 06 & $29.7 \%$ & China 60 & $33.1 \%$ & China 81 & $30.0 \%$ \\
\hline & 5 & China 67 & $24.7 \%$ & China 60 & $39.3 \%$ & China 32 & $20.2 \%$ & China 60 & $29.7 \%$ & China 61 & $29.2 \%$ & Japan 01 & $30.0 \%$ \\
\hline \multirow{5}{*}{ Results - Mix } & 1 & China 67 & 78.7 & China 57 & 61.7 & China 35 & 86.5 & China 13 & 79.2 & China 57 & 72.9 & |Japan 05 & 62.8 \\
\hline & 2 & China 36 & 81.0 & China 67 & 62.7 & China 37 & 86.5 & D052 & 79.3 & China 67 & 73.5 & China 60 & 68.0 \\
\hline & 3 & China 17 & 82.3 & China 60 & 67.8 & China 56 & 87.2 & G072 & 80.2 & China 60 & 75.2 & |Japan 01 & 70.9 \\
\hline & 4 & China 35 & 83.1 & China 56 & 69.4 & China 67 & 87.3 & Japan 01 & 80.8 & Japan 05 & 76.3 & China 68 & 71.9 \\
\hline & 5 & China 37 & 83.1 & Japan 01 & 70.3 & China 32 & 88.6 & C046 & 82.0 & China 58 & 77.2 & China 81 & 72.2 \\
\hline \multicolumn{14}{|c|}{ Drudge 2} \\
\hline Site/results & & \multicolumn{2}{|c|}{ Wackersdorf } & \multicolumn{2}{|c|}{ Horní Bříza } & \multicolumn{2}{|c|}{ Randeck Maar } & \multicolumn{2}{|c|}{ Parschlug } & \multicolumn{2}{|c|}{ Wieliczka } & \multicolumn{2}{|c|}{ Gdów Bay } \\
\hline \multirow{5}{*}{$\begin{array}{l}\text { Results - IPR } \\
\text { Similarity }\end{array}$} & 1 & China 38 & 5.5 & China 87 & 18.0 & D042 & 13.3 & G032 & 17.1 & China 38 & 5.2 & G017 & 10.7 \\
\hline & 2 & Japan 04 & 6.1 & China 65 & 18.9 & G076 & 17.7 & G071 & 21.5 & China 59 & 11.0 & G018 & 11.1 \\
\hline & 3 & China 35 & 7.4 & F075 & 20.4 & D002 & 18.3 & D064 & 22.5 & Japan 04 & 12.5 & F171 & 12.8 \\
\hline & 4 & China 37 & 7.4 & China 63 & 20.6 & D064 & 19.9 & D042 & 24.2 & China 67 & 14.4 & F075 & 12.8 \\
\hline & 5 & China 36 & 10.1 & China 59 & 20.9 & China 38 & 20.2 & G030 & 24.3 & China 35 & 15.2 & G056 & 13.6 \\
\hline \multirow{5}{*}{$\begin{array}{l}\text { Results - } \\
\text { Taxonomic } \\
\text { Similarity }\end{array}$} & 1 & Japan 06 & $31.0 \%$ & China 57 & $46.4 \%$ & China 57 & $23.8 \%$ & China 68 & $32.8 \%$ & Japan 06 & $39.2 \%$ & Japan 05 & $37.5 \%$ \\
\hline & 2 & Japan 05 & $29.1 \%$ & China 67 & $42.9 \%$ & Japan 05 & $21.4 \%$ & China 81 & $31.3 \%$ & Japan 05 & $37.7 \%$ & China 60 & $32.5 \%$ \\
\hline & 3 & China 57 & $27.8 \%$ & China 68 & $39.3 \%$ & China 56 & $21.4 \%$ & Japan 05 & $29.7 \%$ & China 57 & $36.2 \%$ & China 68 & $30.0 \%$ \\
\hline & 4 & China 60 & $27.8 \%$ & China 78 & $39.3 \%$ & China 68 & $20.2 \%$ & Japan 06 & $29.7 \%$ & China 60 & $33.1 \%$ & China 81 & $30.0 \%$ \\
\hline & 5 & China 61 & $27.8 \%$ & China 60 & $39.3 \%$ & China 32 & $20.2 \%$ & China 60 & $29.7 \%$ & China 61 & $29.2 \%$ & Japan 01 & $30.0 \%$ \\
\hline \multirow{5}{*}{ Results - Mix } & 1 & China 67 & 77.7 & China 57 & 58.9 & China 67 & 85.9 & China 68 & 81.2 & China 57 & 71.2 & Japan 05 & 69.7 \\
\hline & 2 & China 60 & 80.8 & China 67 & 61.0 & China 35 & 87.0 & China 38 & 81.6 & China 67 & 73.0 & China 68 & 71.9 \\
\hline & 3 & China 36 & 81.0 & China 60 & 65.3 & China 37 & 87.0 & China 81 & 82.5 & China 60 & 73.6 & China 81 & 71.9 \\
\hline & 4 & China 57 & 81.2 & China 78 & 67.1 & China $57 \mid$ & 87.4 & China 60 & 82.5 & Japan 05 & 74.2 & China 60 & 72.9 \\
\hline & 5 & China 35 & 83.2 & China 56 & 68.9 & China 56 & 88.0 & China 67 & 83.0 & China 58 & 75.7 & China 63 & 75.3 \\
\hline
\end{tabular}


TABLE 2 (continued).

\begin{tabular}{|c|c|c|c|c|c|c|c|c|c|c|c|c|c|}
\hline & & 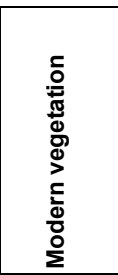 & 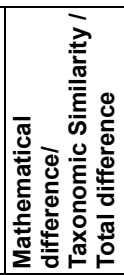 & 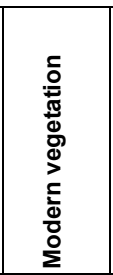 & 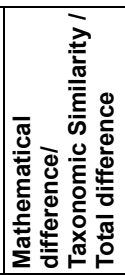 & 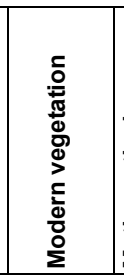 & 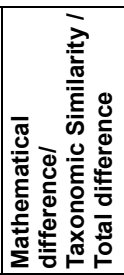 & 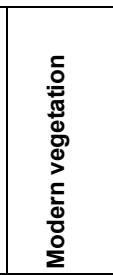 & 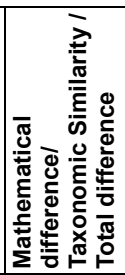 & 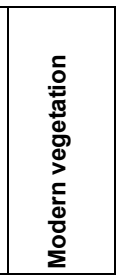 & 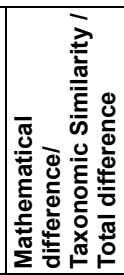 & 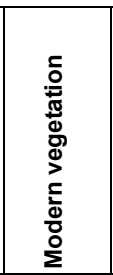 & 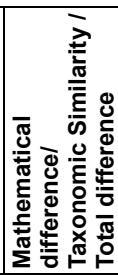 \\
\hline \multicolumn{14}{|c|}{ Drudge 1} \\
\hline Site/results & & \multicolumn{2}{|c|}{ Staré Gliwice } & \multicolumn{2}{|c|}{ Hóly Cross Mts. } & \multicolumn{2}{|c|}{ Erdöbenye } & \multicolumn{2}{|c|}{ Arjuzanx } & \multicolumn{2}{|c|}{ Mataschen } & \multicolumn{2}{|c|}{ Lohnsburg } \\
\hline \multirow{5}{*}{$\begin{array}{l}\text { Results - IPR } \\
\text { Similarity }\end{array}$} & 1 & \begin{tabular}{|l|} 
Japan 01 \\
\end{tabular} & 0.7 & G032 & 1.2 & C009 & 0.2 & D033 & 3.8 & C042 & 5.3 & \begin{tabular}{|l|} 
Japan 02 \\
\end{tabular} & 1.5 \\
\hline & 2 & F054 & 2.5 & G051 & 5.6 & D017 & 1.0 & Japan 17 & 6.8 & C045 & 5.5 & F107 & 1.7 \\
\hline & 3 & F065 & 2.6 & L008 & 5.7 & F010 & 1.4 & D030 & 7.3 & Japan 17 & 7.2 & F129 & 3.1 \\
\hline & 4 & F130 & 2.6 & K016 & 7.1 & F011 & 1.4 & Japan 18 & 8.9 & H001 & 8.5 & Japan 05 & 3.4 \\
\hline & 5 & F131 & 2.7 & J009 & 7.1 & D035 & 1.5 & D061 & 9.3 & F169 & 9.2 & F113 & 3.6 \\
\hline \multirow{5}{*}{$\begin{array}{l}\text { Results - } \\
\text { Taxonomic } \\
\text { Similarity }\end{array}$} & 1 & \begin{tabular}{|l|} 
Japan 06 \\
\end{tabular} & $48.5 \%$ & China 68 & $37.5 \%$ & China 57 & $50.0 \%$ & Japan 05 & $28.4 \%$ & Japan 01 & $25.6 \%$ & China 60 & $60.0 \%$ \\
\hline & 2 & Japan 05 & $46.6 \%$ & China 81 & $35.0 \%$ & China 60 & $48.2 \%$ & Japan 06 & $28.4 \%$ & Japan 06 & $24.8 \%$ & China 67 & $58.6 \%$ \\
\hline & 3 & China 68 & $43.7 \%$ & D052 & $35.0 \%$ & China 61 & $46.4 \%$ & China 60 & $28.4 \%$ & China 60 & $24.1 \%$ & China 61 & $57.1 \%$ \\
\hline & 4 & China 81 & $43.7 \%$ & Japan 05 & $32.5 \%$ & China 68 & $46.4 \%$ & China 57 & $27.2 \%$ & China 68 & $21.1 \%$ & China 68 & $55.7 \%$ \\
\hline & 5 & Japan 01 & $40.8 \%$ & China 61 & $32.5 \%$ & China 78 & $46.4 \%$ & China 61 & $26.0 \%$ & China 81 & $21.1 \%$ & Japan 06 & $54.3 \%$ \\
\hline \multirow{5}{*}{ Results - Mix } & 1 & Japan 05 & 55.5 & D052 & 75.0 & Japan 01 & 60.0 & China 67 & 80.2 & China 36 & 85.0 & China 60 & 41.3 \\
\hline & 2 & Japan 06 & 56.1 & $\mathrm{H} 002$ & 77.7 & China 57 & 65.1 & China 36 & 80.7 & Japan 17 & 85.3 & China 61 & 43.8 \\
\hline & 3 & Japan 01 & 59.2 & $\mathrm{C} 013$ & 78.8 & China 60 & 66.3 & China 38 & 84.1 & China 38 & 85.5 & China 68 & 46.4 \\
\hline & 4 & China 68 & 62.3 & F033 & 79.5 & $\mathrm{H} 002$ & 66.7 & Japan 17 & 84.9 & China 67 & 85.6 & Japan 06 & 46.7 \\
\hline & 5 & China 81 & 62.9 & F139 & 80.0 & Japan 03 & 66.8 & China 60 & 85.1 & China 32 & 86.0 & China 57 & 48.1 \\
\hline \multicolumn{14}{|c|}{ Drudge 2} \\
\hline Site/r & & \multicolumn{2}{|c|}{ Staré Gliwice } & \multicolumn{2}{|c|}{ Holy Cross Mts. } & \multicolumn{2}{|c|}{ Erdöbenye } & \multicolumn{2}{|c|}{ Arjuzanx } & \multicolumn{2}{|c|}{ Mataschen } & \multicolumn{2}{|c|}{ Lohnsburg } \\
\hline \multirow{5}{*}{$\begin{array}{l}\text { Results - IPR } \\
\text { Similarity }\end{array}$} & 1 & F075 & 10.5 & G032 & 1.1 & G032 & 15.5 & China 38 & 13.0 & China 89 & 15.2 & China 79 & 5.3 \\
\hline & 2 & China 87 & 10.6 & G071 & 27.2 & G071 & 19.8 & Japan 04 & 17.5 & China 17 & 19.0 & China 63 & 6.1 \\
\hline & 3 & F171 & 11.6 & J009 & 30.6 & D064 & 21.5 & China 36 & 18.1 & D002 & 20.0 & China 62 & 9.7 \\
\hline & 4 & G017 & 14.6 & G036 & 30.8 & G036 & 21.9 & China 37 & 19.6 & China 38 & 20.1 & China 57 & 11.1 \\
\hline & 5 & G036 & 14.9 & G051 & 31.8 & F075 & 21.9 & China 35 & 19.6 & F169 & 20.9 & China 60 & 11.3 \\
\hline \multirow{5}{*}{$\begin{array}{l}\text { Results - } \\
\text { Taxonomic } \\
\text { Similarity }\end{array}$} & 1 & Japan 06 & $48.5 \%$ & China 68 & $37.5 \%$ & China 57 & $50.0 \%$ & Japan 05 & $28.4 \%$ & Japan 01 & $25.6 \%$ & China 60 & $60.0 \%$ \\
\hline & 2 & Japan 05 & $46.6 \%$ & China 81 & $35.0 \%$ & China 60 & $48.2 \%$ & Japan 06 & $28.4 \%$ & Japan 06 & $24.8 \%$ & China 67 & $58.6 \%$ \\
\hline & 3 & China 68 & $43.7 \%$ & D052 & $35.0 \%$ & China 61 & $46.4 \%$ & China 60 & $28.4 \%$ & China 60 & $24.1 \%$ & China 61 & $57.1 \%$ \\
\hline & 4 & China 81 & $43.7 \%$ & Japan 05 & $32.5 \%$ & China 68 & $46.4 \%$ & China 57 & $27.2 \%$ & China 68 & $21.1 \%$ & China 68 & $55.7 \%$ \\
\hline & 5 & Japan 01 & $40.8 \%$ & China 61 & $32.5 \%$ & China 78 & $46.4 \%$ & China 61 & $26.0 \%$ & China 81 & $21.1 \%$ & Japan 06 & $54.3 \%$ \\
\hline \multirow{5}{*}{ Results - Mix } & 1 & Japan 05 & 59.8 & China 68 & 80.8 & China 57 & 62.2 & China 67 & 78.5 & Japan 01 & 84.1 & China 60 & 41.6 \\
\hline & 2 & China 81 & 60.4 & D052 & 82.8 & China 60 & 63.5 & China 36 & 80.8 & China 17 & 86.3 & China 61 & 45.9 \\
\hline & 3 & China 68 & 61.0 & China 81 & 82.9 & \begin{tabular}{|l|} 
China 67 \\
\end{tabular} & 65.8 & China 60 & 82.1 & F033 & 86.7 & China 67 & 46.4 \\
\hline & 4 & Japan 06 & 61.6 & Japan 05 & 83.2 & \begin{tabular}{|l|} 
China 78 \\
\end{tabular} & 66.9 & China 57 & 83.5 & China 67 & 86.7 & China 57 & 48.4 \\
\hline & 5 & China 57 & 66.0 & D064 & 84.0 & China 61 & 67.0 & China 38 & 83.9 & Japan 02 & 87.3 & China 63 & 49.0 \\
\hline
\end{tabular}


TABLE 2 (continued).

\begin{tabular}{|c|c|c|c|c|c|c|c|c|c|c|c|c|c|}
\hline & & 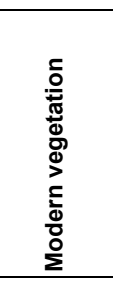 & 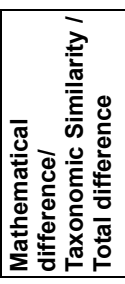 & 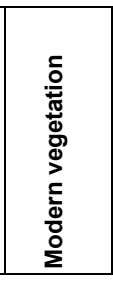 & 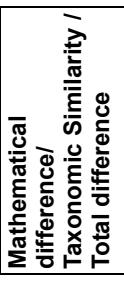 & 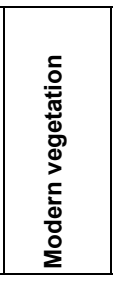 & 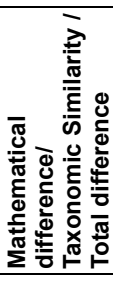 & 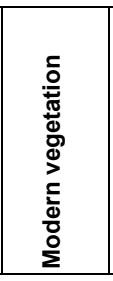 & 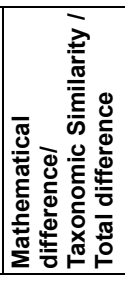 & 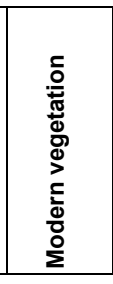 & 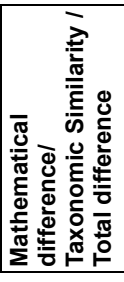 & 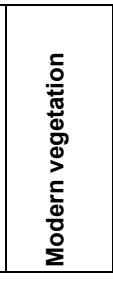 & 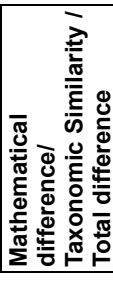 \\
\hline \multicolumn{14}{|c|}{ Drudge 1} \\
\hline Site/results & & \multicolumn{2}{|c|}{$\begin{array}{c}\begin{array}{c}\text { Poštorná-Moravská } \\
\text { Nová Ves }\end{array} \\
\end{array}$} & \multicolumn{2}{|c|}{ Sośnica } & \multicolumn{2}{|c|}{ Ruszów } & \multicolumn{2}{|c|}{ Sessenheim } & \multicolumn{2}{|c|}{ Auenheim } & \multicolumn{2}{|c|}{ Tachov graben } \\
\hline \multirow{5}{*}{$\begin{array}{l}\text { Results - IPR } \\
\text { Similarity }\end{array}$} & 1 & F143 & 1.0 & F130 & 2.0 & F037 & 0.9 & $\begin{array}{c}\text { Mongolia } \\
1\end{array}$ & 3.3 & F159 & 2.0 & F157 & 1.4 \\
\hline & 2 & $\mathrm{H} 002$ & 1.3 & Japan 01 & 2.7 & F157 & 1.2 & F112 & 3.8 & F113 & 2.5 & F117 & 2.8 \\
\hline & 3 & F138 & 1.8 & F054 & 3.3 & F150 & 1.8 & China 56 & 6.0 & F047 & 2.6 & F037 & 3.4 \\
\hline & 4 & F033 & 1.9 & G038 & 3.9 & F104 & 2.7 & China 88 & 6.4 & Japan 05 & 2.7 & F123 & 3.7 \\
\hline & 5 & $\mathrm{H} 003$ & 3.9 & F065 & 4.5 & F117 & 2.7 & F069 & 6.5 & F083 & 3.2 & F150 & 4.0 \\
\hline \multirow{5}{*}{$\begin{array}{c}\text { Results - } \\
\text { Taxonomic } \\
\text { Similarity }\end{array}$} & 1 & Japan 06 & $48.8 \%$ & Japan 06 & $45.3 \%$ & China 67 & $33.9 \%$ & Japan 05 & $36.5 \%$ & China 67 & $56.0 \%$ & Japan 06 & $47.2 \%$ \\
\hline & 2 & China 61 & $46.3 \%$ & China 68 & $44.2 \%$ & Japan 05 & $33.1 \%$ & Japan 06 & $36.5 \%$ & China 81 & $52.0 \%$ & China 81 & $41.7 \%$ \\
\hline & 3 & China 68 & $46.3 \%$ & Japan 01 & $42.1 \%$ & Japan 06 & $33.1 \%$ & Japan 01 & $35.6 \%$ & China 57 & $52.0 \%$ & $\begin{array}{c}\text { Mongolia } \\
1\end{array}$ & $38.9 \%$ \\
\hline & 4 & China 81 & $43.9 \%$ & China 60 & $41.1 \%$ & China 60 & $32.3 \%$ & China 57 & $33.7 \%$ & China 61 & $52.0 \%$ & China 63 & $38.9 \%$ \\
\hline & 5 & Japan 01 & $43.9 \%$ & China 81 & $40.0 \%$ & China 68 & $32.3 \%$ & China 61 & $33.7 \%$ & China 63 & $50.7 \%$ & China 68 & $36.1 \%$ \\
\hline \multirow{5}{*}{ Results - Mix } & 1 & Japan 01 & 58.2 & Japan 01 & 58.0 & Japan 05 & 67.6 & Japan 01 & 66.1 & China 81 & 49.7 & Japan 06 & 54.9 \\
\hline & 2 & China 61 & 60.3 & Japan 06 & 59.6 & Japan 01 & 68.0 & China $67 \mid$ & 67.1 & China 61 & 50.2 & China 63 & 61.7 \\
\hline & 3 & China 56 & 60.3 & China 60 & 61.0 & China 60 & 68.6 & Japan 05 & 67.3 & China 57 & 50.4 & China 81 & 61.9 \\
\hline & 4 & China 62 & 61.7 & China 68 & 62.4 & Japan 06 & 68.9 & China 57 & 67.7 & China 63 & 50.9 & $\begin{array}{c}\text { Mongolia } \\
1\end{array}$ & 62.3 \\
\hline & 5 & China 57 & 62.3 & |Japan 05 & 65.2 & China 57 & 70.1 & China 61 & 67.9 & Japan 05 & 52.1 & Japan 05 & 64.5 \\
\hline \multicolumn{14}{|c|}{ Drudge 2} \\
\hline Site/results & & $\begin{array}{r}\text { Poštorná } \\
\text { Nov }\end{array}$ & $\begin{array}{l}\text {-Moravská } \\
\text { á Ves }\end{array}$ & Sos & śnica & Rus & szów & Sesse & enheim & Aue & nheim & Tacho & graben \\
\hline \multirow{5}{*}{$\begin{array}{l}\text { Results - IPR } \\
\text { Similarity }\end{array}$} & 1 & China 65 & 16.3 & F075 & 10.1 & G018 & 8.9 & China 65 & 14.0 & Japan 05 & 8.6 & D063 & 10.8 \\
\hline & 2 & China 87 & 16.6 & F171 & 10.6 & G017 & 9.1 & F075 & 14.7 & China 79 & 8.7 & D013 & 11.6 \\
\hline & 3 & China 59 & 16.7 & China 87 & 12.1 & F075 & 9.9 & China 59 & 15.7 & China 78 & 9.7 & China 85 & 11.7 \\
\hline & 4 & F075 & 17.1 & G018 & 14.4 & F171 & 10.5 & China $67 \mid$ & 16.1 & China 63 & 11.4 & C017 & 12.2 \\
\hline & 5 & China 67 & 19.0 & G017 & 14.5 & F063 & 10.5 & China 58 & 17.2 & China 57 & 13.1 & D054 & 12.4 \\
\hline \multirow{5}{*}{$\begin{array}{l}\text { Results - } \\
\text { Taxonomic } \\
\text { Similarity }\end{array}$} & 1 & Japan 06 & $48.8 \%$ & |Japan $06 \mid$ & $45.3 \%$ & China 67 & $33.9 \%$ & |Japan $05 \mid$ & $36.5 \%$ & China 67 & $56.0 \%$ & |Japan 06 & $47.2 \%$ \\
\hline & 2 & China 61 & $46.3 \%$ & China 68 & $44.2 \%$ & Japan 05 & $33.1 \%$ & Japan 06 & $36.5 \%$ & China 81 & $52.0 \%$ & China 81 & $41.7 \%$ \\
\hline & 3 & China 68 & $46.3 \%$ & |Japan $01 \mid$ & $42.1 \%$ & Japan 06 & $33.1 \%$ & |Japan $01 \mid$ & $35.6 \%$ & China 57 & $52.0 \%$ & Mongolia & $38.9 \%$ \\
\hline & 4 & China 81 & $43.9 \%$ & China 60 & $41.1 \%$ & China 60 & $32.3 \%$ & China 57 & $33.7 \%$ & China 61 & $52.0 \%$ & China 63 & $38.9 \%$ \\
\hline & 5 & Japan 01 & $43.9 \%$ & China 81 & $40.0 \%$ & China 68 & $32.3 \%$ & China 61 & $33.7 \%$ & China 63 & $50.7 \%$ & China 68 & $36.1 \%$ \\
\hline \multirow{5}{*}{ Results - Mix } & 1 & China 62 & 60.1 & China 68 & 60.4 & China 81 & 69.2 & China 67 & 68.3 & China 57 & 49.7 & $\begin{array}{c}\text { Mongolia } \\
1\end{array}$ & 63.7 \\
\hline & 2 & China 57 & 61.4 & China 60 & 63.3 & China 68 & 69.5 & Japan 05 & 69.2 & China 63 & 50.6 & China 13 & 65.8 \\
\hline & 3 & China 56 & 61.4 & Japan 06 & 63.7 & China 67 & 71.7 & China 57 & 69.8 & China 61 & 50.7 & D011 & 66.1 \\
\hline & 4 & China 67 & 61.5 & China 81 & 63.7 & China 63 & 73.1 & China 68 & 71.2 & China 67 & 51.3 & China 81 & 68.4 \\
\hline & 5 & China 61 & 61.7 & China 71 & 64.4 & China 60 & 73.9 & China 61 & 71.7 & Japan 05 & 52.7 & China 83 & 68.9 \\
\hline
\end{tabular}


TABLE 2 (continued).

\begin{tabular}{|c|c|c|c|c|c|c|c|c|c|c|c|c|c|}
\hline & & 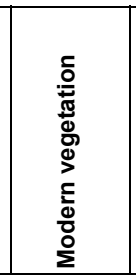 & 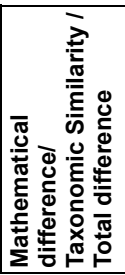 & 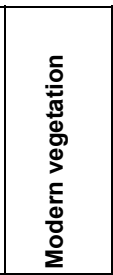 & 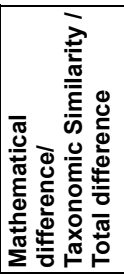 & 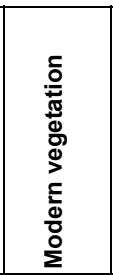 & 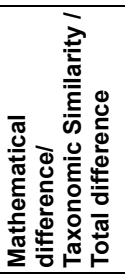 & 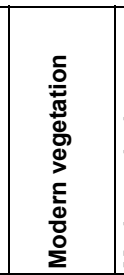 & 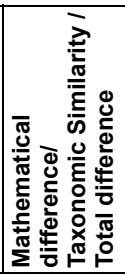 & 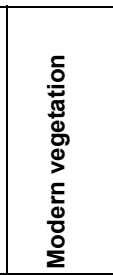 & 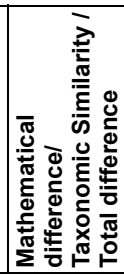 & 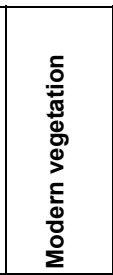 & 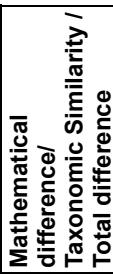 \\
\hline \multicolumn{14}{|c|}{ Drudge 1} \\
\hline Site/results & \multicolumn{3}{|c|}{ Kaltensundheim } & \multicolumn{2}{|c|}{$\begin{array}{c}\text { Vildstejn Fm., Pluto } \\
\text { Clay }\end{array}$} & \multicolumn{2}{|c|}{$\begin{array}{c}\text { Vildstejn Fm., Nero } \\
\text { Clay }\end{array}$} & \multicolumn{2}{|c|}{ Gerstungen } & \multicolumn{2}{|c|}{ Kranichfeld } & \multicolumn{2}{|c|}{ Berga } \\
\hline \multirow{5}{*}{$\begin{array}{l}\text { Results - IPR } \\
\text { Similarity }\end{array}$} & 1 & F119 & 0.6 & China 04 & 1.7 & F123 & 0.5 & China 56 & 0.4 & China 59 & 2.7 & F123 & 1.3 \\
\hline & 2 & China 63 & 2.2 & F123 & 4.6 & F150 & 2.7 & China 88 & 2.4 & F146 & 3.5 & F157 & 1.8 \\
\hline & 3 & China 79 & 3.6 & China 62 & 4.8 & F157 & 3.0 & China 65 & 4.8 & China 67 & 3.6 & F150 & 2.1 \\
\hline & 4 & China 85 & 4.4 & China 80 & 4.8 & F037 & 3.4 & China 58 & 5.5 & China 66 & 3.7 & F037 & 2.5 \\
\hline & 5 & China 61 & 5.2 & China 60 & 5.2 & F142 & 3.5 & $\begin{array}{c}\text { Mongolia } \\
2\end{array}$ & 6.3 & $\begin{array}{c}\text { Mongolia } \\
2\end{array}$ & 5.0 & F104 & 3.9 \\
\hline \multirow{5}{*}{$\begin{array}{l}\text { Results - } \\
\text { Taxonomic } \\
\text { Similarity }\end{array}$} & 1 & F033 & $45.9 \%$ & Japan 05 & $29.0 \%$ & China 68 & $33.7 \%$ & Japan 05 & $53.8 \%$ & China 68 & $48.6 \%$ & Japan 01 & $33.8 \%$ \\
\hline & 2 & China 68 & $43.2 \%$ & Japan 06 & $28.0 \%$ & Japan 06 & $30.2 \%$ & Japan 06 & $48.7 \%$ & $\begin{array}{c}\text { Mongolia } \\
1\end{array}$ & $48.6 \%$ & F033 & $33.8 \%$ \\
\hline & 3 & F024 & $43.2 \%$ & China 68 & $28.0 \%$ & China 81 & $29.1 \%$ & $\begin{array}{c}\text { Mongolia } \\
1\end{array}$ & $43.6 \%$ & F013 & $45.9 \%$ & F035 & $33.8 \%$ \\
\hline & 4 & Mongolia 1 & $40.5 \%$ & China 81 & $27.0 \%$ & Japan 05 & $29.1 \%$ & Japan 01 & $43.6 \%$ & Japan 05 & $43.2 \%$ & China 68 & $32.3 \%$ \\
\hline & 5 & F013 & $40.5 \%$ & F033 & $26.0 \%$ & F033 & $27.9 \%$ & China 68 & $41.0 \%$ & China 71 & $43.2 \%$ & F031 & $32.3 \%$ \\
\hline \multirow{5}{*}{ Results - Mix } & 1 & China 68 & 58.4 & Japan 05 & 72.3 & China 68 & 70.2 & Japan 05 & 51.8 & $\begin{array}{c}\text { Mongolia } \\
1\end{array}$ & 52.9 & Japan 01 & 66.5 \\
\hline & 2 & F033 & 61.6 & Japan 06 & 74.6 & Japan 05 & 72.0 & $\begin{array}{c}\text { Mongolia } \\
1\end{array}$ & 57.1 & F013 & 64.5 & F035 & 66.6 \\
\hline & 3 & Mongolia 1 & 62.2 & China 61 & 75.3 & Japan 06 & 72.3 & |Japan 06 & 58.6 & D011 & 64.7 & F031 & 68.3 \\
\hline & 4 & China 81 & 63.9 & China 68 & 75.7 & Japan 01 & 73.6 & Japan 01 & 59.7 & Japan 05 & 65.4 & F033 & 68.6 \\
\hline & 5 & F035 & 64.0 & $\begin{array}{c}\text { Mongolia } \\
1\end{array}$ & 76.4 & F033 & 74.1 & China 67 & 62.1 & China 68 & 66.2 & China 68 & 71.2 \\
\hline \multicolumn{14}{|c|}{ Drudge 2} \\
\hline Site/results & & \multicolumn{2}{|c|}{ Kaltensundheim } & \multicolumn{2}{|c|}{\begin{tabular}{|c|} 
Vildstejn Fm., Pluto \\
Clay
\end{tabular}} & \multicolumn{2}{|c|}{$\begin{array}{c}\text { Vildstejn Fm., Nero } \\
\text { Clay }\end{array}$} & \multicolumn{2}{|c|}{ Gerstungen } & \multicolumn{2}{|c|}{ Kranichfeld } & \multicolumn{2}{|c|}{ Berga } \\
\hline \multirow{5}{*}{$\begin{array}{l}\text { Results - IPR } \\
\text { Similarity }\end{array}$} & 1 & D041 & 6.9 & F054 & 9.0 & $\mathrm{C} 037$ & 5.9 & China 88 & 11.9 & D011 & 11.1 & G038 & 5.4 \\
\hline & 2 & G001 & 7.3 & G055 & 9.3 & F130 & 6.6 & China 85 & 14.8 & D013 & 11.8 & F054 & 6.8 \\
\hline & 3 & F152 & 7.7 & G038 & 9.5 & F054 & 6.6 & G056 & 17.5 & D030 & 12.3 & F016 & 6.9 \\
\hline & 4 & D058 & 8.1 & F069 & 9.6 & China 83 & 8.4 & D064 & 17.5 & D016 & 13.5 & F069 & 7.3 \\
\hline & 5 & F064 & 8.1 & C037 & 9.7 & F045 & 9.0 & G055 & 17.5 & D063 & 13.5 & F053 & 7.9 \\
\hline \multirow{5}{*}{$\begin{array}{l}\text { Results - } \\
\text { Taxonomic } \\
\text { Similarity }\end{array}$} & 1 & F033 & $45.9 \%$ & Japan 05 & $29.0 \%$ & China 68 & $33.7 \%$ & Japan 05 & $53.8 \%$ & China 68 & $48.6 \%$ & Japan 01 & $33.8 \%$ \\
\hline & 2 & China 68 & $43.2 \%$ & Japan 06 & $28.0 \%$ & Japan 06 & $30.2 \%$ & Japan 06 & $48.7 \%$ & $\begin{array}{c}\text { Mongolia } \\
1\end{array}$ & $48.6 \%$ & F033 & $33.8 \%$ \\
\hline & 3 & F024 & $43.2 \%$ & China 68 & $28.0 \%$ & China 81 & $29.1 \%$ & $\begin{array}{c}\text { Mongolia } \\
1\end{array}$ & $43.6 \%$ & F013 & $45.9 \%$ & F035 & $33.8 \%$ \\
\hline & 4 & Mongolia 1 & $40.5 \%$ & China 81 & $27.0 \%$ & Japan 05 & $29.1 \%$ & Japan 01 & $43.6 \%$ & Japan 05 & $43.2 \%$ & China 68 & $32.3 \%$ \\
\hline & 5 & F013 & $40.5 \%$ & F033 & $26.0 \%$ & F033 & $27.9 \%$ & China 68 & $41.0 \%$ & China 71 & $43.2 \%$ & F031 & $32.3 \%$ \\
\hline \multirow{5}{*}{ Results - Mix } & 1 & F033 & 56.0 & China 68 & 75.0 & China 68 & 70.2 & |Japan $05 \mid$ & 65.1 & $\begin{array}{c}\text { Mongolia } \\
1\end{array}$ & 55.2 & F033 & 68.7 \\
\hline & 2 & F024 & 59.2 & China 81 & 75.5 & F033 & 73.9 & China 85 & 65.8 & D011 & 60.5 & Japan 01 & 69.8 \\
\hline & 3 & Mongolia 1 & 60.5 & F033 & 76.4 & China 81 & 74.3 & China 68 & 66.2 & F013 & 61.9 & China 68 & 69.9 \\
\hline & 4 & F035 & 62.9 & China 71 & 79.4 & Japan 01 & 76.0 & China 81 & 66.4 & D016 & 63.6 & F031 & 70.3 \\
\hline & 5 & F013 & 63.0 & Japan 01 & 79.4 & D052 & 77.3 & Japan 01 & 67.8 & China 68 & 64.1 & China 81 & 71.0 \\
\hline
\end{tabular}


TABLE 2 (continued).

\begin{tabular}{|c|c|c|c|c|c|c|c|c|c|c|c|c|c|}
\hline & & 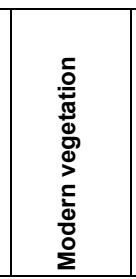 & 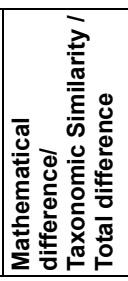 & 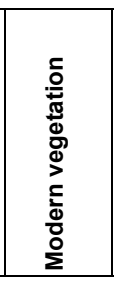 & 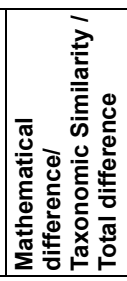 & 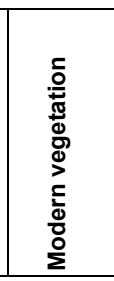 & 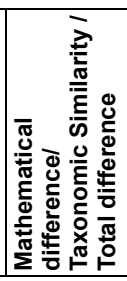 & 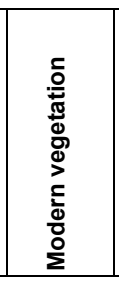 & 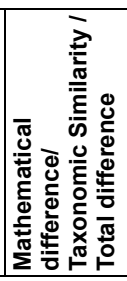 & 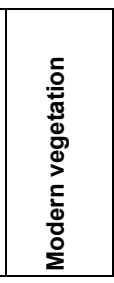 & 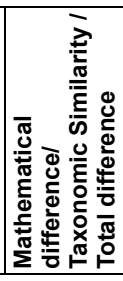 & 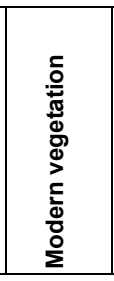 & 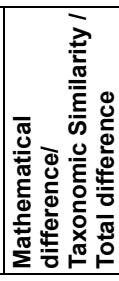 \\
\hline \multicolumn{14}{|c|}{ Drudge 1} \\
\hline Site/results & & \multicolumn{2}{|c|}{$\begin{array}{c}\text { Vildstejn Fm., lignite } \\
\text { beds }\end{array}$} & \multicolumn{2}{|c|}{ Rippersroda } & \multicolumn{2}{|c|}{$\begin{array}{l}\text { Vildstejn Fm., } \\
\text { upper beds }\end{array}$} & \multicolumn{2}{|c|}{ Nordhausen } & \multicolumn{2}{|c|}{ Klinger beds } & \multicolumn{2}{|c|}{ Güvem } \\
\hline \multirow{5}{*}{$\begin{array}{l}\text { Results - IPR } \\
\text { Similarity }\end{array}$} & 1 & F142 & 2.9 & China 78 & 2.4 & F157 & 1.4 & China 04 & 3.0 & China 78 & 1.2 & $\mathrm{C008}$ & 0.5 \\
\hline & 2 & F155 & 3.6 & China 77 & 2.6 & F117 & 2.8 & $\mid \begin{array}{c}\text { Mongolia } \\
1\end{array}$ & 3.8 & F144 & 4.9 & F001 & 1.3 \\
\hline & 3 & F069 & 4.1 & Japan 06 & 3.5 & F037 & 3.4 & F142 & 4.7 & F119 & 5.7 & D056 & 1.9 \\
\hline & 4 & Mongolia 1 & 5.0 & China 71 & 4.3 & F123 & 3.7 & F123 & 6.0 & China 83 & 5.8 & D042 & 3.1 \\
\hline & 5 & F130 & 5.7 & F144 & 4.8 & F150 & 4.0 & China 65 & 6.3 & Japan 06 & 5.9 & F030 & 3.3 \\
\hline \multirow{5}{*}{$\begin{array}{l}\text { Results - } \\
\text { Taxonomic } \\
\text { Similarity }\end{array}$} & 1 & Japan 06 & $31.3 \%$ & China 68 & $57.6 \%$ & Japan 06 & $47.2 \%$ & F031 & $40.9 \%$ & Japan 05 & $75.0 \%$ & Japan 01 & $26.0 \%$ \\
\hline & 2 & Mongolia 1 & $31.3 \%$ & China 81 & $45.5 \%$ & China 81 & $41.7 \%$ & F033 & $31.8 \%$ & China 68 & $75.0 \%$ & Japan 05 & $26.0 \%$ \\
\hline & 3 & China 81 & $29.9 \%$ & $\begin{array}{c}\text { Mongolia } \\
1\end{array}$ & $42.4 \%$ & $\begin{array}{c}\text { Mongolia } \\
1\end{array}$ & $38.9 \%$ & F042 & $31.8 \%$ & F043 & $68.8 \%$ & China 57 & $26.0 \%$ \\
\hline & 4 & Japan 01 & $28.4 \%$ & F043 & $42.4 \%$ & China 63 & $38.9 \%$ & Japan 01 & $31.8 \%$ & China 13 & $68.8 \%$ & Japan 06 & $25.0 \%$ \\
\hline & 5 & China 68 & $28.4 \%$ & Japan 06 & $42.4 \%$ & China 68 & $36.1 \%$ & China 80 & $31.8 \%$ & China 71 & $65.6 \%$ & China 60 & $24.0 \%$ \\
\hline \multirow{5}{*}{ Results - Mix } & 1 & Mongolia 1 & 68.8 & China 68 & 42.7 & Japan 06 & 54.9 & F031 & 61.3 & Japan 05 & 26.0 & F007 & 83.9 \\
\hline & 2 & Japan 01 & 72.0 & China 81 & 54.9 & China 63 & 61.7 & China 80 & 68.7 & China 68 & 26.4 & Japan 17 & 86.1 \\
\hline & 3 & Japan 06 & 73.1 & Japan 06 & 57.7 & China 81 & 61.9 & Japan 01 & 69.1 & F043 & 35.2 & Japan 01 & 86.2 \\
\hline & 4 & F033 & 74.0 & F043 & 59.9 & $\begin{array}{c}\text { Mongolia } \\
1\end{array}$ & 62.3 & F033 & 70.2 & China 71 & 35.2 & China 67 & 87.1 \\
\hline & 5 & Japan 05 & 75.4 & $\begin{array}{c}\text { Mongolia } \\
1\end{array}$ & 63.3 & Japan 05 & 64.5 & F042 & 70.8 & China 81 & 35.7 & China 12 & 87.1 \\
\hline \multicolumn{14}{|c|}{ Drudge 2} \\
\hline Site/results & & \multicolumn{2}{|c|}{\begin{tabular}{|c|} 
Vildstejn Fm., lignite \\
beds
\end{tabular}} & \multicolumn{2}{|c|}{ Rippersroda } & \multicolumn{2}{|c|}{$\begin{array}{c}\text { Vildstejn Fm., } \\
\text { upper beds }\end{array}$} & \multicolumn{2}{|c|}{ Nordhausen } & \multicolumn{2}{|c|}{ Klinger beds } & \multicolumn{2}{|c|}{ Güvem } \\
\hline \multirow{5}{*}{$\begin{array}{l}\text { Results - IPR } \\
\text { Similarity }\end{array}$} & 1 & $\mathrm{C} 017$ & 6.3 & F165 & 8.8 & D063 & 10.8 & F022 & 7.0 & F171 & 15.0 & D042 & 16.0 \\
\hline & 2 & D013 & 8.5 & F029 & 9.2 & D013 & 11.6 & F050 & 7.4 & G017 & 15.2 & D002 & 20.8 \\
\hline & 3 & $\mathrm{~F} 130$ & 9.4 & F047 & 9.8 & China 85 & 11.7 & G001 & 7.9 & China 87 & 15.5 & G076 & 23.1 \\
\hline & 4 & China 13 & 9.5 & D039 & 9.9 & $\mathrm{C} 017$ & 12.2 & F152 & 7.9 & China 63 & 15.9 & China 38 & 23.8 \\
\hline & 5 & $\mathrm{C} 013$ & 9.8 & F046 & 10.1 & D054 & 12.4 & D041 & 8.0 & China 81 & 16.2 & D045 & 24.4 \\
\hline \multirow{5}{*}{$\begin{array}{l}\text { Results - } \\
\text { Taxonomic } \\
\text { Similarity }\end{array}$} & 1 & Japan 06 & $31.3 \%$ & China 68 & $57.6 \%$ & Japan 06 & $47.2 \%$ & F031 & $40.9 \%$ & Japan 05 & $75.0 \%$ & Japan 01 & $26.0 \%$ \\
\hline & 2 & Mongolia 1 & $31.3 \%$ & China 81 & $45.5 \%$ & China 81 & $41.7 \%$ & F033 & $31.8 \%$ & China 68 & $75.0 \%$ & Japan 05 & $26.0 \%$ \\
\hline & 3 & China 81 & $29.9 \%$ & $\begin{array}{c}\text { Mongolia } \\
1\end{array}$ & $42.4 \%$ & $\begin{array}{c}\text { Mongolia } \\
1\end{array}$ & $38.9 \%$ & F042 & $31.8 \%$ & F043 & $68.8 \%$ & China 57 & $26.0 \%$ \\
\hline & 4 & Japan 01 & $28.4 \%$ & F043 & $42.4 \%$ & China 63 & $38.9 \%$ & Japan 01 & $31.8 \%$ & China 13 & $68.8 \%$ & Japan 06 & $25.0 \%$ \\
\hline & 5 & China 68 & $28.4 \%$ & Japan 06 & $42.4 \%$ & China 68 & $36.1 \%$ & China 80 & $31.8 \%$ & China 71 & $65.6 \%$ & China 60 & $24.0 \%$ \\
\hline \multirow{5}{*}{ Results - Mix } & 1 & Mongolia 1 & 70.2 & China 68 & 46.6 & $\begin{array}{c}\text { Mongolia } \\
1\end{array}$ & 63.7 & F031 & 61.9 & China 68 & 32.5 & China 67 & 84.5 \\
\hline & 2 & Japan 01 & 73.9 & China 81 & 55.5 & China 13 & 65.8 & China 80 & 68.7 & Japan 05 & 34.3 & China 59 & 85.5 \\
\hline & 3 & F033 & 74.7 & F043 & 58.6 & D011 & 66.1 & F042 & 68.9 & China 81 & 38.0 & China 57 & 87.5 \\
\hline & 4 & China 81 & 77.1 & $\begin{array}{c}\text { Mongolia } \\
1\end{array}$ & 66.0 & China 81 & 68.4 & Japan 01 & 69.3 & China 71 & 38.5 & F007 & 87.6 \\
\hline & 5 & F043 & 77.6 & China 13 & 66.0 & China 83 & 68.9 & F024 & 69.7 & China 63 & 40.7 & China 58 & 87.9 \\
\hline
\end{tabular}


TABLE 2 (continued).

\begin{tabular}{|c|c|c|c|c|c|c|c|c|c|c|c|c|c|}
\hline & & 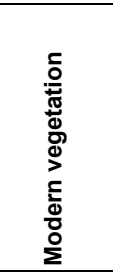 & 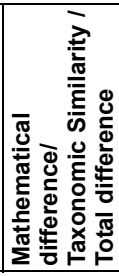 & 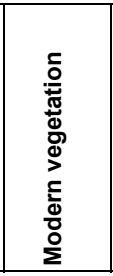 & 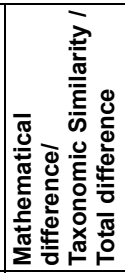 & 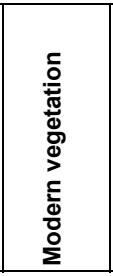 & 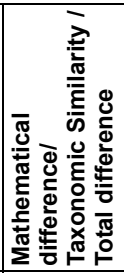 & 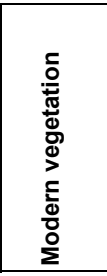 & 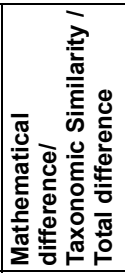 & 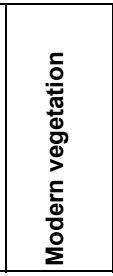 & 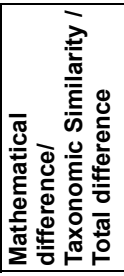 & 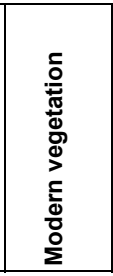 & 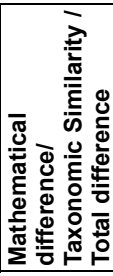 \\
\hline \multicolumn{14}{|c|}{ Drudge 1} \\
\hline Site/results & & \multicolumn{2}{|c|}{ Kymi } & \multicolumn{2}{|c|}{ Yatagan Basin } & \multicolumn{2}{|c|}{ Pitsidia } & \multicolumn{2}{|c|}{ Mt. Tondo } & \multicolumn{2}{|c|}{ Tossignano } & \multicolumn{2}{|c|}{ Ca' Viettone } \\
\hline \multirow{5}{*}{$\begin{array}{l}\text { Results - IPR } \\
\text { Similarity }\end{array}$} & 1 & K027 & 3.1 & F019 & 1.4 & G066 & 1.2 & F026 & 1.6 & D016 & 0.7 & C045 & 2.0 \\
\hline & 2 & C038 & 4.3 & China 14 & 1.4 & G014 & 1.2 & D030 & 1.8 & F136 & 2.3 & C042 & 2.1 \\
\hline & 3 & F023 & 6.9 & D054 & 2.0 & F002 & 1.5 & D011 & 2.5 & D011 & 2.9 & Japan 04 & 6.3 \\
\hline & 4 & G042 & 7.2 & G030 & 2.0 & F008 & 1.5 & F136 & 4.1 & K009 & 3.7 & China 38 & 6.4 \\
\hline & 5 & C019 & 8.0 & G041 & 3.0 & F082 & 1.6 & K009 & 4.6 & F026 & 4.4 & H001 & 6.6 \\
\hline \multirow{5}{*}{$\begin{array}{c}\text { Results - } \\
\text { Taxonomic } \\
\text { Similarity }\end{array}$} & 1 & China 68 & $27.9 \%$ & China 57 & $40.6 \%$ & China 61 & $36.4 \%$ & Japan 05 & $33.9 \%$ & China 57 & $29.9 \%$ & Japan 06 & $34.8 \%$ \\
\hline & 2 & China 81 & $26.2 \%$ & China 61 & $39.1 \%$ & China 62 & $36.4 \%$ & Japan 06 & $33.9 \%$ & China 61 & $29.9 \%$ & Japan 05 & $34.2 \%$ \\
\hline & 3 & Japan 05 & $26.2 \%$ & China 67 & $39.1 \%$ & China 63 & $34.1 \%$ & China 61 & $33.9 \%$ & China 67 & $28.7 \%$ & Japan 01 & $28.4 \%$ \\
\hline & 4 & Japan 06 & $24.6 \%$ & China 68 & $37.5 \%$ & China 67 & $34.1 \%$ & China 68 & $33.9 \%$ & Japan 05 & $27.6 \%$ & China 60 & $27.1 \%$ \\
\hline & 5 & China 32 & $24.6 \%$ & China 77 & $35.9 \%$ & China 68 & $34.1 \%$ & China 57 & $32.3 \%$ & Japan 06 & $27.6 \%$ & China 68 & $26.5 \%$ \\
\hline \multirow{5}{*}{ Results - Mix } & 1 & F009 & 85.6 & $\mathrm{H} 002$ & 69.6 & Japan 01 & 69.4 & China 67 & 74.3 & China 67 & 76.7 & China 17 & 78.0 \\
\hline & 2 & China 38 & 86.0 & Japan 01 & 71.1 & China 62 & 72.7 & China 56 & 78.0 & China 56 & 79.4 & China 67 & 79.1 \\
\hline & 3 & D016 & 86.1 & China 67 & 71.3 & D052 & 72.7 & China 36 & 78.6 & China 13 & 81.4 & |Japan $17 \mid$ & 79.5 \\
\hline & 4 & G072 & 87.0 & C046 & 71.9 & China 61 & 73.4 & Japan 01 & 78.8 & China 36 & 81.4 & Japan 15 & 80.8 \\
\hline & 5 & F007 & 87.3 & China 12 & 72.3 & China $67 \mid$ & 73.5 & F162 & 78.9 & China 57 & 81.4 & China 36 & 81.5 \\
\hline \multicolumn{14}{|c|}{ Drudge 2} \\
\hline Site/results & & \multicolumn{2}{|c|}{ Kymi } & \multicolumn{2}{|c|}{ Yatagan Basin } & \multicolumn{2}{|c|}{ Pitsidia } & \multicolumn{2}{|c|}{ Mt. Tondo } & \multicolumn{2}{|c|}{ Tossignano } & \multicolumn{2}{|c|}{$\mathrm{Ca}^{\prime}$ Viettone } \\
\hline \multirow{5}{*}{$\begin{array}{l}\text { Results - IPR } \\
\text { Similarity }\end{array}$} & 1 & D042 & 17.0 & G032 & 17.0 & G032 & 19.7 & D042 & 17.1 & D042 & 12.4 & China 38 & 14.4 \\
\hline & 2 & D002 & 21.2 & G071 & 22.8 & F075 & 20.0 & China 38 & 18.3 & G076 & 12.6 & China 89 & 15.1 \\
\hline & 3 & D045 & 22.4 & F075 & 24.1 & China 87 & 20.2 & China 59 & 21.3 & K009 & 12.8 & |Japan 04 & 18.4 \\
\hline & 4 & J048 & 23.1 & D064 & 24.8 & G036 & 20.7 & D002 & 22.0 & D064 & 13.3 & D042 & 19.3 \\
\hline & 5 & J009 & 24.1 & G036 & 25.5 & G071 & 20.9 & D064 & 22.8 & G041 & 13.8 & China 59 & 19.5 \\
\hline \multirow{5}{*}{$\begin{array}{l}\text { Results - } \\
\text { Taxonomic } \\
\text { Similarity }\end{array}$} & 1 & China 68 & $27.9 \%$ & China 57 & $40.6 \%$ & China 61 & $36.4 \%$ & Japan 05 & $33.9 \%$ & China 57 & $29.9 \%$ & Japan 06 & $34.8 \%$ \\
\hline & 2 & China 81 & $26.2 \%$ & China 61 & $39.1 \%$ & China 62 & $36.4 \%$ & Japan 06 & $33.9 \%$ & China 61 & $29.9 \%$ & Japan 05 & $34.2 \%$ \\
\hline & 3 & Japan 05 & $26.2 \%$ & China 67 & $39.1 \%$ & China 63 & $34.1 \%$ & China 61 & $33.9 \%$ & China 67 & $28.7 \%$ & Japan 01 & $28.4 \%$ \\
\hline & 4 & Japan 06 & $24.6 \%$ & China 68 & $37.5 \%$ & China 67 & $34.1 \%$ & China 68 & $33.9 \%$ & Japan 05 & $27.6 \%$ & China 60 & $27.1 \%$ \\
\hline & 5 & China 32 & $24.6 \%$ & China 77 & $35.9 \%$ & China 68 & $34.1 \%$ & China 57 & $32.3 \%$ & Japan 06 & $27.6 \%$ & China 68 & $26.5 \%$ \\
\hline \multirow{5}{*}{ Results - Mix } & 1 & China 38 & 84.2 & China 67 & 68.6 & China 62 & 69.7 & China 67 & 72.7 & China 67 & 78.2 & China 17 & 78.2 \\
\hline & 2 & Japan 03 & 87.2 & China 57 & 70.2 & China 63 & 71.0 & China 62 & 77.0 & Japan 03 & 79.5 & China 67 & 79.6 \\
\hline & 3 & China 36 & 87.9 & China 63 & 72.2 & China 67 & 71.5 & China 59 & 77.2 & China 59 & 82.5 & Japan 05 & 82.7 \\
\hline & 4 & China 32 & 89.6 & China 56 & 73.9 & China 57 & 72.8 & China 63 & 77.8 & China 68 & 82.5 & China 36 & 83.3 \\
\hline & 5 & China 68 & 89.6 & China 61 & 74.0 & China 61 & 72.9 & China 36 & 78.7 & China 63 & 82.5 & China 60 & 83.7 \\
\hline
\end{tabular}


TABLE 2 (continued).

\begin{tabular}{|c|c|c|c|c|c|c|c|c|c|c|c|c|c|}
\hline & & 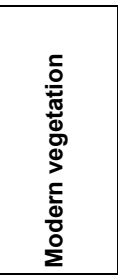 & 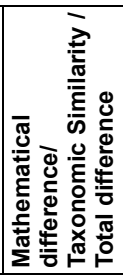 & 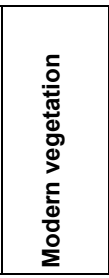 & 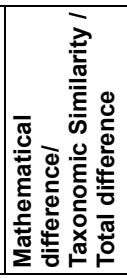 & 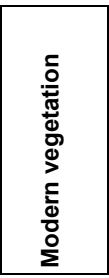 & 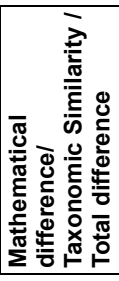 & 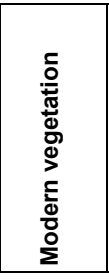 & 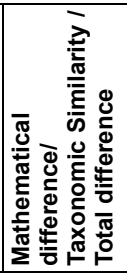 & 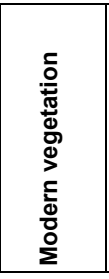 & 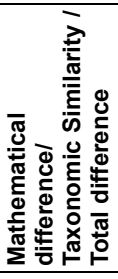 & 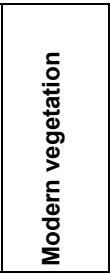 & 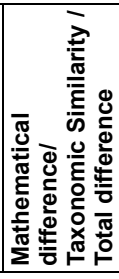 \\
\hline \multicolumn{14}{|c|}{ Drudge 1} \\
\hline Site/results & & \multicolumn{2}{|c|}{ Shamb 1b+A1 } & \multicolumn{2}{|c|}{ Shamb 1f+B1 } & \multicolumn{2}{|c|}{ Darbas 2/d } & \multicolumn{2}{|c|}{$\begin{array}{c}\text { Uyts-2/a + Uts-2 } \\
\text { PZ23 }\end{array}$} & \multicolumn{2}{|c|}{$\begin{array}{c}\text { Darbas-2/f + Drb-2 } \\
\text { PZ6 }\end{array}$} & \multicolumn{2}{|c|}{ Tolors 2} \\
\hline \multirow{5}{*}{$\begin{array}{l}\text { Results - IPR } \\
\text { Similarity }\end{array}$} & 1 & D055 & 1.6 & G028 & 1.9 & G040 & 1.7 & G022 & 0.8 & F022 & 1.0 & F035 & 0.4 \\
\hline & 2 & F090 & 2.0 & $\mathrm{C} 044$ & 2.0 & D028 & 2.0 & G019 & 1.5 & F068 & 1.1 & G018 & 1.4 \\
\hline & 3 & F018 & 2.2 & D012 & 2.4 & G023 & 2.4 & G020 & 1.6 & D041 & 1.2 & F063 & 1.7 \\
\hline & 4 & D036 & 2.6 & G060 & 2.6 & F153 & 2.8 & L001 & 1.9 & G056 & 1.3 & F031 & 1.8 \\
\hline & 5 & China 10 & 2.7 & F092 & 2.8 & F094 & 2.8 & G010 & 2.4 & F029 & 1.4 & F028 & 1.9 \\
\hline \multirow{5}{*}{$\begin{array}{l}\text { Results - } \\
\text { Taxonomic } \\
\text { Similarity }\end{array}$} & 1 & Japan 06 & $26.5 \%$ & China 71 & $53.3 \%$ & China 71 & $65.4 \%$ & China 71 & $48.2 \%$ & Japan 06 & $54.1 \%$ & China 71 & $33.3 \%$ \\
\hline & 2 & China 71 & $24.5 \%$ & China 68 & $50.7 \%$ & China 68 & $56.8 \%$ & China 68 & $46.5 \%$ & China 81 & $49.2 \%$ & F013 & $31.4 \%$ \\
\hline & 3 & Japan 05 & $24.5 \%$ & Japan 05 & $49.3 \%$ & China 81 & $55.6 \%$ & Japan 05 & $43.0 \%$ & Japan 05 & $49.2 \%$ & L014 & $29.4 \%$ \\
\hline & 4 & L014 & $22.4 \%$ & Japan 06 & $45.4 \%$ & Japan 01 & $55.6 \%$ & China 78 & $42.1 \%$ & China 68 & $44.3 \%$ & China 60 & $27.5 \%$ \\
\hline & 5 & China 63 & $22.4 \%$ & F073 & $45.4 \%$ & Japan 06 & $54.3 \%$ & China 81 & $41.2 \%$ & China 71 & $44.3 \%$ & China 63 & $27.5 \%$ \\
\hline \multirow{5}{*}{ Results - Mix } & 1 & L014 & 81.1 & D052 & 57.6 & Japan 01 & 45.5 & China 71 & 61.2 & Japan 06 & 49.5 & China 71 & 69.5 \\
\hline & 2 & Japan 05 & 81.9 & Japan 05 & 59.0 & China 71 & 46.0 & Japan 05 & 61.6 & Japan 05 & 52.2 & L014 & 70.7 \\
\hline & 3 & F033 & 82.4 & F073 & 59.8 & D052 & 47.4 & China 68 & 63.2 & China 81 & 56.2 & D052 & 73.1 \\
\hline & 4 & $\mathrm{H} 002$ & 82.8 & $\mathrm{C} 046$ & 60.4 & F031 & 48.2 & D052 & 64.0 & F043 & 59.3 & Japan 05 & 73.1 \\
\hline & 5 & Japan 06 & 82.9 & F033 & 60.6 & F032 & 49.2 & F043 & 65.1 & China 71 & 59.8 & F013 & 73.4 \\
\hline \multicolumn{14}{|c|}{ Drudge 2} \\
\hline Site/results & & \multicolumn{2}{|c|}{ Shamb $1 b+A 1$} & \multicolumn{2}{|c|}{ Shamb 1f+B1 } & \multicolumn{2}{|c|}{ Darbas 2/d } & \multicolumn{2}{|c|}{$\begin{array}{c}\text { Uyts-2/a + Uts-2 } \\
\text { PZ23 }\end{array}$} & \multicolumn{2}{|c|}{$\begin{array}{c}\text { Darbas-2/f + Drb-2 } \\
\text { PZ6 }\end{array}$} & \multicolumn{2}{|c|}{ Tolors 2} \\
\hline \multirow{5}{*}{$\begin{array}{l}\text { Results - IPR } \\
\text { Similarity }\end{array}$} & 1 & G040 & 7.8 & G071 & 6.8 & G036 & 3.9 & G058 & 4.5 & China 83 & 8.7 & D053 & 5.9 \\
\hline & 2 & $\mathrm{C} 047$ & 8.1 & G036 & 7.2 & G008 & 7.8 & G062 & 5.4 & G055 & 9.2 & D018 & 7.2 \\
\hline & 3 & G039 & 8.9 & G060 & 7.9 & F171 & 8.1 & G010 & 5.8 & G039 & 9.6 & G004 & 8.4 \\
\hline & 4 & G019 & 9.2 & G058 & 8.0 & F052 & 9.2 & G024 & 6.0 & G056 & 10.1 & $\mathrm{C} 046$ & 8.7 \\
\hline & 5 & D053 & 9.5 & G010 & 8.8 & G018 & 9.4 & G025 & 6.8 & China 85 & 10.2 & F018 & 8.8 \\
\hline \multirow{5}{*}{$\begin{array}{l}\text { Results - } \\
\text { Taxonomic } \\
\text { Similarity }\end{array}$} & 1 & Japan 06 & $26.5 \%$ & China 71 & $53.3 \%$ & China 71 & $65.4 \%$ & China 71 & $48.2 \%$ & Japan 06 & $54.1 \%$ & China 71 & $33.3 \%$ \\
\hline & 2 & China 71 & $24.5 \%$ & China 68 & $50.7 \%$ & China 68 & $56.8 \%$ & China 68 & $46.5 \%$ & China 81 & $49.2 \%$ & F013 & $31.4 \%$ \\
\hline & 3 & Japan 05 & $24.5 \%$ & Japan 05 & $49.3 \%$ & China 81 & $55.6 \%$ & Japan 05 & $43.0 \%$ & Japan 05 & $49.2 \%$ & L014 & $29.4 \%$ \\
\hline & 4 & L014 & $22.4 \%$ & Japan 06 & $45.4 \%$ & Japan 01 & $55.6 \%$ & China 78 & $42.1 \%$ & China 68 & $44.3 \%$ & China 60 & $27.5 \%$ \\
\hline & 5 & China 63 & $22.4 \%$ & F073 & $45.4 \%$ & Japan 06 & $54.3 \%$ & China 81 & $41.2 \%$ & China 71 & $44.3 \%$ & China 63 & $27.5 \%$ \\
\hline \multirow{5}{*}{ Results - Mix } & 1 & L014 & 78.5 & China 71 & 52.4 & China 71 & 39.1 & China 71 & 56.4 & China 81 & 58.3 & L014 & 71.8 \\
\hline & 2 & China 71 & 83.1 & China 68 & 54.7 & China 68 & 47.3 & China 68 & 57.2 & China 68 & 60.9 & F013 & 72.2 \\
\hline & 3 & G056 & 83.6 & F073 & 60.7 & China 81 & 49.2 & China 81 & 63.8 & China 71 & 62.2 & D052 & 74.0 \\
\hline & 4 & $\mathrm{H} 002$ & 84.7 & D052 & 60.8 & G022 & 53.1 & D052 & 65.6 & F043 & 62.4 & L006 & 75.4 \\
\hline & 5 & G037 & 85.0 & China 81 & 61.1 & F032 & 53.2 & F043 & 66.5 & China 13 & 65.1 & C043 & 75.8 \\
\hline
\end{tabular}


similarity approaches is $1350(54 \times 5 \times 5)$. Figures 2 and 3 show these results summarised to vegetation types for East Asia and vegetation formations for Europe.

In the sections Results and Discussion, numbers are rounded to 1 except for values $<1$, which are provided to the decimal. East Asian vegetation types and European vegetation formations are treated from south to north and within the respective types/formations according to their relevance based on score results, i.e., numbers of proxies delivered by the Drudges. East Asian vegetation types as summarised in this study are indicated by quotation marks (see Table 3). For Figures 2 and 3 we recommend viewing Appendix 4 because of the interactive colour signature there. By moving the cursor over the colour columns the designation of the proxies and their relevance are provided for every fossil assemblage.

\section{Reference Set of Modern Vegetation}

Summaries of modern vegetation from East Asia and Europe are available in great detail (legend Appendix 5). For Europe, the vegetation units are assigned to vegetation formations according to Bohn et al. (2004). The vegetation units from Asia derive from different sources (Wang, 1961; Teodoridis et al., 2011) and were already summarised to vegetation types by Teodoridis et al. (2020, table 3 there) to the extent that they served as proxies for that study. We present a more encompassing scheme in which all units of the reference set of modern vegetation are assigned to vegetation types. Vegetation types as summarised for this study are indicated by quotation marks, e.g., "MMF China" (Table 3).

In Teodoridis et al. (2020) the reference set of modern vegetation included the unit China 03 (see Teodoridis et al., 2020, app. 4, 7). With more than 630 zonal taxa, this unit was by far the most diverse vegetation unit of the modern reference set. Due to its unusual diversity, its discriminating potential was very low, which was already discussed in that paper. To avoid this effect, China 03 was excluded from the reference set of modern vegetation data in the present study (Table 3). Therefore, the modern reference set currently stands at 503 units.

More comprehensive consideration must be given to the Mixed Mesophytic Forest Formation (sensu Wang, 1961). In empirical studies of younger Palaeogene and Neogene plant assemblages, it is the most often proposed modern analogy. Wang (1961) categorised the Mixed
Mesophytic Forest (MMF) Formation regionally into MMF of the Lower Yangtze Provinces (here units China 56 to 61) and the Broad-leaved Deciduous Forests (BLDF) of the Upper Yangtze Provinces (China 62 to 67) (Appendix 5). According to Wang (1961) both forest types represent deciduous broad-leaved forests bearing the richest woody flora of all temperate regions with a wide overlap in genera and species, including numerous relict and endemic taxa. Both types further resemble each other by lacking primary constituents in the tree level, their very rich understories, and the diversity of conifers. Differences mentioned by Wang (1961) refer mainly to the altitudinal distribution and therefore to climatic conditions. The BLDF of the Upper Yangtze Provinces appear at higher elevations than the MMF of the Lower Yangtze Provinces, and the mean annual precipitation - which is usually between 1000 to $1500 \mathrm{~mm}$ in the area of the MMF Formation - reaches up to $2000 \mathrm{~mm}$ at higher elevations. Teodoridis et al. (2020) treated BLDF of Meili Snow Mt. jointly with the BLDF of the Upper Yangtze Provinces (Table 3). Using Wang's data, the IPR vegetation analysis was performed to more precisely characterise these forest types (Appendices 6,7$)$. The results indicate minor differences between MMF Lower Yangtze Provinces and BLDF Upper Yangtze Provinces sensu Wang (1961), whereby the IPR vegetation analysis does not enable a clearer distinction. Comparing the floristic composition based on Wang (1961), the mean number of zonal woody angiosperms is higher in the MMF of the Lower Yangtze Provinces (62) than in the BLDF of the Upper Yangtze Provinces (45). At the same time, the overall number of woody taxa in the latter (210) exceeds that of the former (196). Both vegetation types share many species, but 28 genera are distinctive for the MMF of the Lower Yangtze Provinces and 34 distinguish the BLDF of the Upper Yangtze Provinces from it. Accordingly, the floristic composition is what distinguishes the two, and it is justified to treat them separately even though they cannot be distinguished unambiguously by the IPR vegetation analysis.

To improve the focus on major vegetation types, some types from China and Japan treated separately by Teodoridis et al. (2020) were merged here because major trends may remain masked if the assessment is too small-scaled. These are: "Broad-leaved evergreen forest, China" is fused with "Broad-leaved evergreen forest, Honshu" to "BLEF China, Japan" (Table 3).

The units Japan 01 to 03 and Japan 05, which Teodoridis et al. (2020) treated as a separate vege- 
IPR Similarity Drudge 1 IPR Similarity Drudge 2 Results Mix Drudge 1 Results Mix Drudge 2
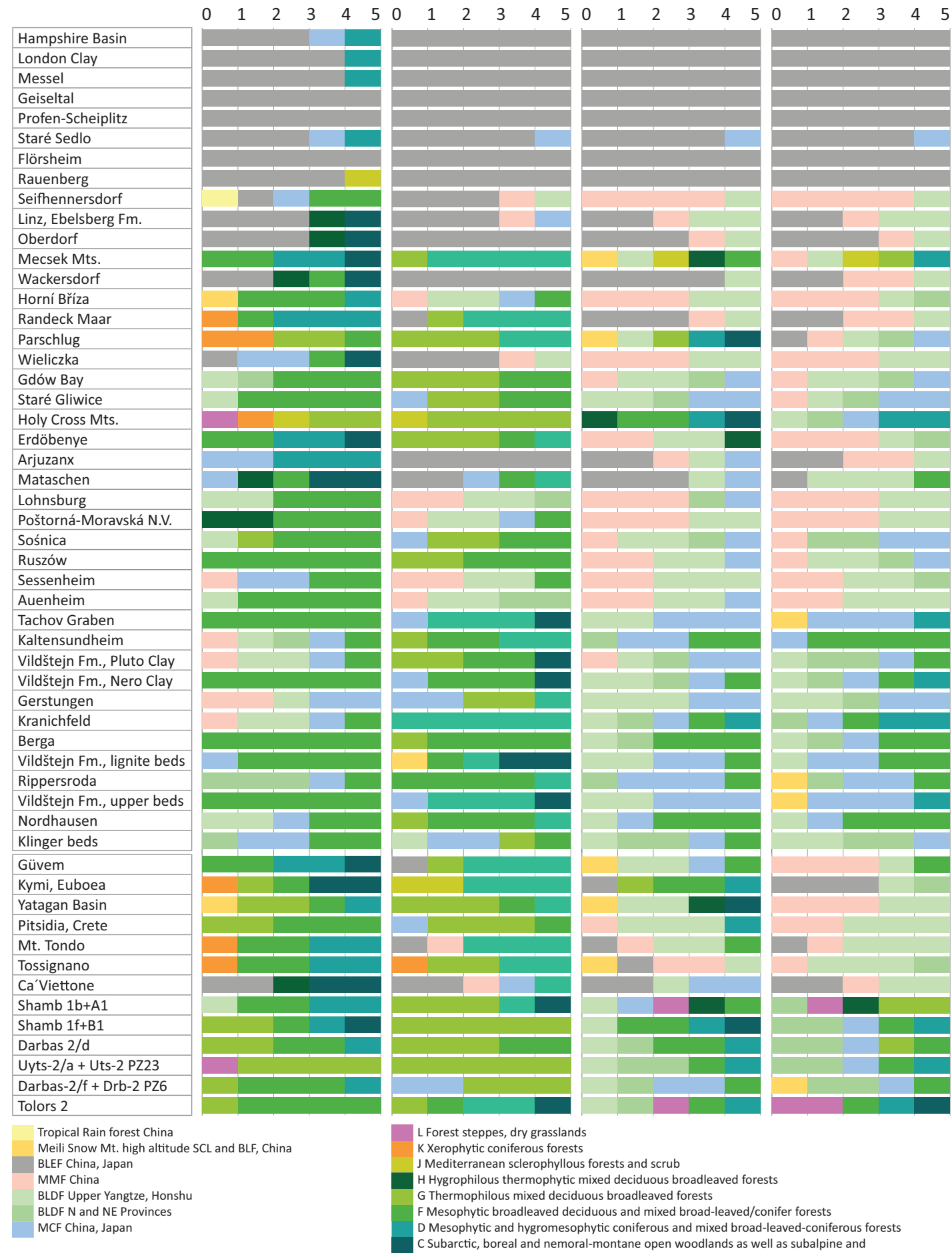

L Forest steppes, dry grasslands

$\mathrm{K}$ Xerophytic coniferous forests

J Mediterranean sclerophyllous forests and scrub

Hygrophilous thermophytic mixed deciduous broadleaved forests

$G$ Thermophilous mixed deciduous broadleaved forests

F Mesophytic broadleaved deciduous and mixed broad-leaved/conifer forests

D Mesophytic and hygromesophytic coniferous and mixed broad-leaved-coniferous forests

C Subarctic, boreal and nemoral-montane open woodlands as well as subalpine and

oro-Mediterranean vegetation

FIGURE 2. Modern vegetation types/formations delivered as proxies by Drudges 1 and 2 for the test set of fossil assemblages. Shown are the five best fitted results for the IPR Similarities based on Drudge 1 and Drudge 2 and for the Results Mix based on Drudge 1 and Drudge 2. Pastel colours represent East Asian vegetation types, bright colours European vegetation formations. For more detailed information see Appendix 4 which provides interactive colour signature (moving the cursor over the columns provides the designation of the proxies and their relevance for every fossil assemblage). 
Taxonomic Similarity Overall scores (synthesis results Drudges 1+2)

\begin{tabular}{|l|}
\hline Hampshire Basin \\
\hline London Clay \\
\hline Messel \\
\hline Geiseltal \\
\hline Profen-Scheiplitz \\
\hline Staré Sedlo \\
\hline Flörsheim \\
\hline Rauenberg \\
\hline Seifhennersdorf \\
\hline Linz, Ebelsberg Fm. \\
\hline Oberdorf \\
\hline Mecsek Mts. \\
\hline Wackersdorf \\
\hline Horní Bř́za \\
\hline Randeck Maar \\
\hline Parschlug \\
\hline Wieliczka \\
\hline Gdów Bay \\
\hline Staré Gliwice \\
\hline Holy Cross Mts. \\
\hline Erdöbenye \\
\hline Arjuzanx \\
\hline Mataschen \\
\hline Lohnsburg \\
\hline Poštorná-Moravská N.V. \\
\hline Sośnica \\
\hline Ruszów \\
\hline Sessenheim \\
\hline Auenheim \\
\hline Tachov Graben \\
\hline Kaltensundheim \\
\hline Vildštejn Fm., Pluto Clay \\
\hline Vildštejn Fm., Nero Clay \\
\hline Gerstungen \\
\hline Kranichfeld \\
\hline Berga \\
\hline Vildštejn Fm., lignite beds \\
\hline Rippersroda \\
\hline Vildštejn Fm., upper beds \\
\hline Nordhausen \\
\hline Klinger beds \\
\hline \hline Güvem \\
\hline Kymi, Euboea \\
\hline Yatagan Basin \\
\hline Pitsidia, Crete \\
\hline Mt. Tondo \\
\hline Tossignano \\
\hline Ca'Viettone \\
\hline Shamb 1b+A1 \\
\hline Shamb 1f+B1 \\
\hline Darbas 2/d \\
\hline Uyts-2/a + Uts-2 PZ23 N and NE Provinces \\
\hline Darbas-2/f + Drb-2 PZ6 \\
\hline Tolors 2 \\
\hline Tropical Rain forest China \\
Meili Snow Mt. high altitude SCL and BLF, China \\
\hline BLFF China, Japan \\
\hline BLF Upper Yante, Honsh \\
\hline
\end{tabular}

0

5

10

15

$20 \quad 25$

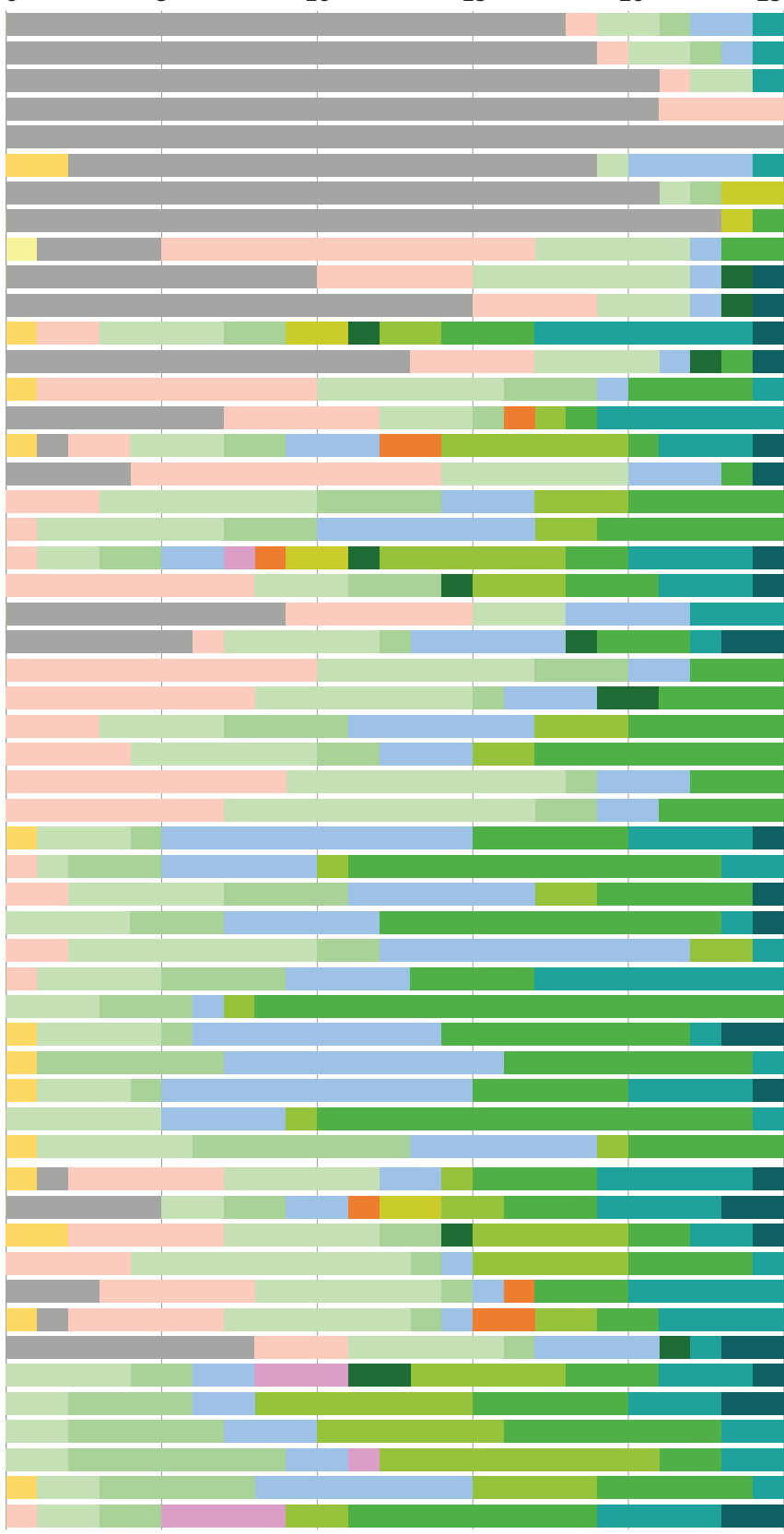

L Forest steppes, dry grasslands

K Xerophytic coniferous forests

J Mediterranean sclerophyllous forests and scrub

H Hygrophilous thermophytic mixed deciduous broadleaved forests

$\mathrm{G}$ Thermophilous mixed deciduous broadleaved forests

G Thermophilous mixed deciduous broadleaved forests

road-leaved/conifer forests

D Mesophytic and hygromesophytic coniferous and mixed broad-leaved-coniferous forests

C Subarctic, boreal and nemoral-montane open woodlands as well as subalpine and oro-Mediterranean vegetation

FIGURE 3. Modern vegetation types/formations delivered as proxies by Drudges 1 and 2 for the test set of fossil assemblages. Shown are the five best fitted results for the Taxonomic Similarity (TS) and the overall scores (synthesis of all similarity approaches), i.e., 25 proxies for every plant assemblage. Pastel colours represent East Asian vegetation types, bright colours European vegetation formations. For more detailed information see Appendix 4 which provides interactive colour signature (moving the cursor over the columns provides the designation of the proxies and their relevance for every fossil assemblage). 
TABLE 3. East Asian vegetation types and European vegetation formations as applied in this study.

\begin{tabular}{|c|c|c|c|c|c|}
\hline & & odoridis et al. (2020) & & this paper & \\
\hline & $\begin{array}{l}\text { vegetation } \\
\text { units (ID } \\
\text { code) }\end{array}$ & vegetation types/formations & $\begin{array}{l}\text { vegetation units } \\
\text { (ID code) }\end{array}$ & vegetation types/formations & acronym \\
\hline & & & China 23-28 & Tropical Rain Forests China & $\begin{array}{l}\text { "Tropical Rain } \\
\text { Forest China" }\end{array}$ \\
\hline & $\begin{array}{l}\text { China } 17,28 \\
30-38\end{array}$ & $\begin{array}{l}\text { Broad-leaved Evergreen Forest, } \\
\text { China }\end{array}$ & $\begin{array}{l}\text { China 01,15-22, } \\
\text { 29-38, Japan 04, }\end{array}$ & $\begin{array}{l}\text { Broad-leaved evergreen forest, } \\
\text { China, Japan }\end{array}$ & $\begin{array}{l}\text { "BLEF China, } \\
\text { Japan" }\end{array}$ \\
\hline & $\begin{array}{l}\text { Japan 04, 07, } \\
08\end{array}$ & $\begin{array}{l}\text { Broad-leaved Evergreen Forest, } \\
\text { Honshu, Japan }\end{array}$ & $07-14$ & & \\
\hline & China 13,14 & $\begin{array}{l}\text { Meili Snow Mt. - Sclerophyllous } \\
\text { and broad-leaved forest zone } \\
(2580-3650 \mathrm{~m} \text { alt })\end{array}$ & China 11- 14 & $\begin{array}{l}\text { Meili Snow Mt. - Sclerophyllous and } \\
\text { broad-leaved forest zone (2580- } \\
3650 \mathrm{~m} \text { alt) }\end{array}$ & $\begin{array}{l}\text { "Meili Snow Mt. } \\
\text { high altitude SCL } \\
\text { and BLF, China" }\end{array}$ \\
\hline & & & China 39-55 & $\begin{array}{l}\text { Grasslands and Desert China, } \\
\text { Mongolia }\end{array}$ & \\
\hline & China 56-61 & $\begin{array}{l}\text { Mixed Mesophytic Forest of the } \\
\text { lower Yangtze Provinces }\end{array}$ & China $02,56-61$ & $\begin{array}{l}\text { Mixed Mesophytic Forest, lower } \\
\text { Yangtze Provinces }\end{array}$ & "MMF China" \\
\hline 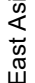 & China 03 & $\begin{array}{l}\text { Mt. Emei evergreen or deciduous } \\
\text { broad-leaved mixed forest zone }\end{array}$ & & $\begin{array}{l}\text { excluded (see section Reference set } \\
\text { of modern vegetation) }\end{array}$ & \\
\hline & $\begin{array}{l}\text { China 04, } \\
62-67\end{array}$ & $\begin{array}{l}\text { Broad-leaved Deciduous Forest, } \\
\text { Upper Yangtze Provinces and Mt. } \\
\text { Emei }\end{array}$ & $\begin{array}{l}\text { China 04-10, 62- } \\
67 \text {, Japan 01- } \\
03,05\end{array}$ & $\begin{array}{l}\text { Broad-leaved Deciduous Forest, } \\
\text { Upper Yangtze Provinces, Mt. Emei, } \\
\text { and Honshu }\end{array}$ & $\begin{array}{l}\text { "BLDF Upper } \\
\text { Yangtze, Honshu" }\end{array}$ \\
\hline & $\begin{array}{l}\text { Japan } \\
01-03,05\end{array}$ & $\begin{array}{l}\text { Broad-leaved deciduous forests } \\
\text { Shirakami Sanchi, and Mt. Fuji }\end{array}$ & & & \\
\hline & China 68-79 & $\begin{array}{l}\text { Broad-leaved Deciduous Forests } \\
\text { of the Northern and Northeastern } \\
\text { Provinces }\end{array}$ & China 68-79 & $\begin{array}{l}\text { Broad-leaved Deciduous Forests of } \\
\text { the Northern and Northeastern } \\
\text { Provinces (China) }\end{array}$ & $\begin{array}{l}\text { "BLDF N and NE } \\
\text { Provinces, China" }\end{array}$ \\
\hline & China 80-89 & $\begin{array}{l}\text { Montane Coniferous Forests, } \\
\text { China, Taiwan }\end{array}$ & $\begin{array}{l}\text { China 80-89, } \\
\text { Japan 06, 15-18 }\end{array}$ & $\begin{array}{l}\text { Montane Coniferous Forest China, } \\
\text { Honshu, Yakushima }\end{array}$ & $\begin{array}{l}\text { "MCF China, } \\
\text { Japan" }\end{array}$ \\
\hline & Japan 06 & $\begin{array}{l}\text { Mt. Fuji-Upper montane and } \\
\text { subalpine coniferous belt, 1800- } \\
2500 \text { m, Vaccinium-Picea region }\end{array}$ & & & \\
\hline
\end{tabular}

tation type, resemble the forests of the MMF Formation in China in their diversity of zonal woody angiosperms and percentage of their BLD component. Japan 05 further resembles those forests by the comparably high number of conifer species. Based on this analysis and the fact that Japan 01 to 03 and 05 are situated further north than the MMF Formation in China, these units from Japan were merged with the BLDF of the Upper Yangtze Provinces (which reach higher altitudes than the MMF Lower Yangtze Provinces) to "BLDF Upper Yangtze Provinces, Honshu" (Table 3). Nonetheless, note that in Japan 01 to 03 and 05 the BLE component is lower and the SCL+LEG component is higher than that of the MMF Lower Yangtze Provinces and the BLDF of the Upper Yangtze Provinces (Appendix 7).

Finally, "Montane Coniferous Forests, China, Taiwan" (China 80 to 89) were merged with Japan
06 and Japan 15 to 18, which represent mountain coniferous forests on Honshu and Yakushima Island (Japan), to "MCF China, Japan" (Table 3, Appendix 5).

The composition of European vegetation formations is available in great detail (Bohn et al., 2004). The formations are split into up to 172 mapping units (Formation F, Appendix 5). Therefore, the Formations C, D, F, and $\mathrm{G}$ were treated according to the subdivisions in Bohn et al. (2004).

\section{RESULTS}

\section{Applying the Drudges}

The proxy sets for all similarity approaches (IPR Similarity, Taxonomic Similarity (TS) and Results Mix derived by both Drudges) are summarised in Table 2, Figures 2, 3, and Appendix 4. 
TABLE 3 (continued).

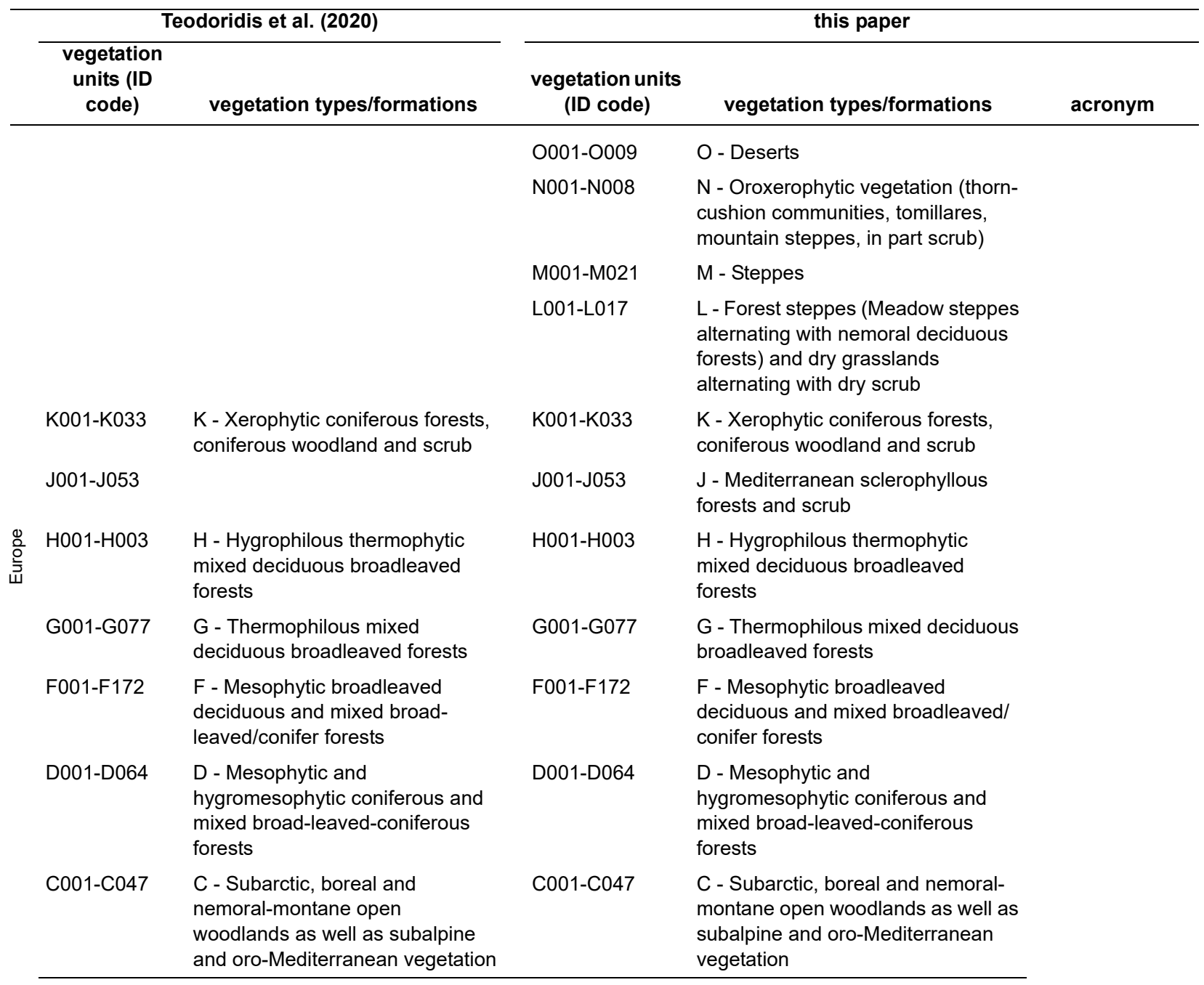

Based on Drudge 1 almost $69 \%$ of the scores there (IPR Similarity, TS, Results Mix) point towards East Asian vegetation types and 31\% towards Europe. In Drudge 2 this relation is $73 \%$ for East Asia and 27\% for Europe. The IPR Similarities of both Drudges indicate both East Asian and European affinities (35\%/65\% in Drudge 1, 43\%/ $57 \%$ in Drudge 2). An East Asian affinity dominates from the Eocene through early Miocene, and then European vegetation formations become increasingly important from the early Miocene onwards (Figure 2). In contrast, the TS points mainly towards East Asian affinity (92\%) (Table 4, Figure $3)$. In the Results Mix of Drudge 1 and 2 the relation East Asian verse European vegetation units are similar $(81 \% / 19 \%$ in the former, $84 \% / 16 \%$ in the latter). In the overall score this relation points almost two thirds towards East Asia and one third to Europe (67\% to $33 \%)$.
The following vegetation types/formations received at least $10 \%$ of the overall scores (Table 4): From Asia these were "BLEF in East Asia" (19\%), "MMF China" (11\%), "BLDF Upper Yangtze, Honshu" (16\%), and "MCF China, Japan" (12\%); from Europe this was only Formation $F(15 \%)$. At least $5 \%$ of the overall scores were obtained by "BLDF N and NE Provinces, China" (8\%), and the European vegetation Formations $\mathrm{G}(6 \%)$ and $\mathrm{D}$ $(7 \%)$. Less than $5 \%$ of the overall scores were tallied for "Tropical Rain Forest China" $(0.1 \%)$,"Meili Snow Mt. high altitude SCL and BLF, China" (1\%), and the European vegetation Formations $L(0.7 \%)$, $\mathrm{K}(0.6 \%), \mathrm{J}(0.7 \%), \mathrm{H}(1 \%)$, and $\mathrm{C}(2 \%)$. The vegetation type "Tropical Rain forest China" $(0.1 \%)$ and the European vegetation Formations $\mathrm{M}$ to $\mathrm{O}$, which did not receive any score, are not discussed here. 
TABLE 4. Distribution of the modern East Asian and European vegetation proxies. More detailed information is available in Appendix 8.

\begin{tabular}{|c|c|c|c|c|c|c|c|c|}
\hline $\begin{array}{c}\text { Vegetation types East } \\
\text { Asia }\end{array}$ & $\begin{array}{l}\text { "Tropical } \\
\text { Rain Forest } \\
\text { China" }\end{array}$ & $\begin{array}{l}\text { "Meili } \\
\text { Snow Mt. } \\
\text { high } \\
\text { altitude } \\
\text { SCL and } \\
\text { BLF, } \\
\text { China" }\end{array}$ & $\begin{array}{l}\text { "BLEF } \\
\text { China, } \\
\text { Japan" }\end{array}$ & $\begin{array}{l}\text { "MMF } \\
\text { China" }\end{array}$ & $\begin{array}{l}\text { "BLDF } \\
\text { Upper } \\
\text { Yangtze, } \\
\text { Honshu" }\end{array}$ & $\begin{array}{l}\text { "BLDF N } \\
\text { and NE } \\
\text { Provinces, } \\
\text { China" }\end{array}$ & $\begin{array}{l}\text { "MCF } \\
\text { China, } \\
\text { Japan" }\end{array}$ & Sum \\
\hline Number of units & 6 & 4 & 28 & 7 & 17 & 12 & 18 & \\
\hline $\begin{array}{l}\text { Number of units } \\
\text { delivered as proxies }\end{array}$ & 1 & 3 & 26 & 6 & 11 & 5 & 14 & \\
\hline $\begin{array}{l}\text { Number of units } \\
\text { delivered as proxies in } \\
\text { percent }\end{array}$ & $17 \%$ & $75 \%$ & $93 \%$ & $86 \%$ & $65 \%$ & $42 \%$ & $78 \%$ & \\
\hline $\begin{array}{l}\text { Scores in the IPR } \\
\text { Similarity, Drudge } 1\end{array}$ & 1 & 2 & 45 & 6 & 15 & 6 & 20 & 95 \\
\hline in percent & $0.4 \%$ & $0.7 \%$ & $17 \%$ & $2 \%$ & $6 \%$ & $2 \%$ & $7 \%$ & $35 \%$ \\
\hline $\begin{array}{l}\text { Scores in the IPR } \\
\text { Similarity, Drudge } 2\end{array}$ & 0 & 1 & 70 & 12 & 13 & 3 & 18 & 117 \\
\hline in percent & $0.0 \%$ & $0.4 \%$ & $26 \%$ & $4 \%$ & $5 \%$ & $1 \%$ & $7 \%$ & $43 \%$ \\
\hline Scores in the TS & 0 & 3 & 18 & 54 & 68 & 49 & 56 & 248 \\
\hline in percent & $0.0 \%$ & $1.1 \%$ & $7 \%$ & $20 \%$ & $25 \%$ & $18 \%$ & $21 \%$ & $92 \%$ \\
\hline $\begin{array}{l}\text { Scores in the Results } \\
\text { Mix, Drudge } 1\end{array}$ & 0 & 5 & 61 & 35 & 64 & 17 & 37 & 219 \\
\hline in percent & $0.0 \%$ & $2 \%$ & $23 \%$ & $13 \%$ & $24 \%$ & $6 \%$ & $14 \%$ & $81 \%$ \\
\hline $\begin{array}{l}\text { Scores in the Results } \\
\text { Mix, Drudge } 2\end{array}$ & 0 & 4 & 58 & 48 & 55 & 30 & 32 & 227 \\
\hline in percent & $0.0 \%$ & $1.5 \%$ & $21 \%$ & $18 \%$ & $20 \%$ & $11 \%$ & $12 \%$ & $84 \%$ \\
\hline $\begin{array}{l}\text { Scores IPR Similarity, } \\
\text { TS, Results Mix in } \\
\text { Drudge } 1\end{array}$ & 1 & 10 & 124 & 95 & 147 & 72 & 113 & 562 \\
\hline $\begin{array}{l}\text { in percent of the overall } \\
\text { scores }\end{array}$ & $0.1 \%$ & $0.7 \%$ & $9 \%$ & $7 \%$ & $11 \%$ & $5 \%$ & $8 \%$ & $42 \%$ \\
\hline $\begin{array}{l}\text { Scores IPR Similarity, } \\
\text { TS, Results Mix in } \\
\text { Drudge } 2\end{array}$ & 0 & 8 & 146 & 114 & 136 & 82 & 106 & 592 \\
\hline $\begin{array}{l}\text { in percent of the overall } \\
\text { scores }\end{array}$ & $0.0 \%$ & $0.6 \%$ & $11 \%$ & $8 \%$ & $10 \%$ & $6 \%$ & $8 \%$ & $44 \%$ \\
\hline $\begin{array}{l}\text { Overall scores IPR } \\
\text { Similarity, TS, Results } \\
\text { Mix, both Drudges }\end{array}$ & 1 & 15 & 252 & 155 & 215 & 105 & 163 & 906 \\
\hline in percent & $0.1 \%$ & $1 \%$ & $19 \%$ & $11 \%$ & $16 \%$ & $8 \%$ & $12 \%$ & $67 \%$ \\
\hline
\end{tabular}

\section{East Asian Vegetation Types}

"Meili Snow Mt. high altitude SCL and BLF, China". The scores of this vegetation type are distributed among three of its four units (Table 4). This vegetation type occurs sporadically only in the similarity approaches (IPR Similarity, Taxonomic Similarity (TS), Results Mix of both Drudges), with scores between 0.4 and almost $2 \%$. In the overall score it accounts for 1\% (Figure 4). The scores are spread from the Eocene (Staré Sedlo) to the Miocene (Horní Bríza, Yatagan Basin), Pliocene (Vildštejn Fm.), and Pleistocene (Klinger beds) across the similarity approaches (Figure 5).

"BLEF China, Japan". This type comprises 28 vegetation units of which $93 \%$ (26) are delivered as proxies (Table 4). Nineteen percent of the overall scores are allotted here (Figure 4). "BLEF China, Japan" is delivered as a proxy in the IPR Similarity 
TABLE 4 (continued).

\begin{tabular}{|c|c|c|c|c|c|c|c|c|c|c|}
\hline Vegetation formations Europe & $\mathbf{L}$ & $\mathbf{K}$ & $\mathbf{J}$ & $\mathbf{H}$ & G & $\mathbf{F}$ & D & C & $\begin{array}{l}\text { Sum of } \\
\text { scores } \\
\text { Europe }\end{array}$ & Sum \\
\hline Number of units & 17 & 33 & 53 & 3 & 77 & 172 & 64 & 47 & & \\
\hline Number of units delivered as proxies & 4 & 5 & 6 & 3 & 35 & 77 & 26 & 13 & & \\
\hline $\begin{array}{l}\text { Number of units delivered as proxies in } \\
\text { percent }\end{array}$ & $24 \%$ & $15 \%$ & $11 \%$ & $100 \%$ & $45 \%$ & $45 \%$ & $41 \%$ & $28 \%$ & & \\
\hline Scores in the IPR Similarity, Drudge 1 & 2 & 7 & 2 & 7 & 20 & 96 & 27 & 14 & 175 & 175 \\
\hline in percent & $0.7 \%$ & $3 \%$ & $0.7 \%$ & $3 \%$ & $7 \%$ & $36 \%$ & $10 \%$ & $5 \%$ & $65 \%$ & $65 \%$ \\
\hline Scores in the IPR Similarity, Drudge 2 & 0 & 1 & 3 & 0 & 58 & 39 & 44 & 8 & 153 & 153 \\
\hline in percent & $0.0 \%$ & $0.4 \%$ & $1.1 \%$ & $0.0 \%$ & $21 \%$ & $14 \%$ & $16 \%$ & $3 \%$ & $57 \%$ & $57 \%$ \\
\hline Scores in the TS & 2 & 0 & 2 & 0 & 0 & 17 & 1 & 0 & 22 & 22 \\
\hline in percent & $0.7 \%$ & $0.0 \%$ & $0.7 \%$ & $0.0 \%$ & $0.0 \%$ & $6 \%$ & $0.4 \%$ & $0.0 \%$ & $8 \%$ & $8 \%$ \\
\hline Scores in the Results Mix, Drudge 1 & 2 & 0 & 1 & 5 & 2 & 28 & 9 & 4 & 51 & 51 \\
\hline in percent & $0.7 \%$ & $0.0 \%$ & $0.4 \%$ & $2 \%$ & $0.7 \%$ & $10 \%$ & $3 \%$ & $1.5 \%$ & $19 \%$ & $19 \%$ \\
\hline Scores in the Results Mix, Drudge 2 & 3 & 0 & 1 & 1 & 4 & 22 & 11 & 1 & 43 & 43 \\
\hline in percent & $1.1 \%$ & $0.0 \%$ & $0.4 \%$ & $0.4 \%$ & $1.5 \%$ & $8 \%$ & $4 \%$ & $0.4 \%$ & $16 \%$ & $16 \%$ \\
\hline $\begin{array}{l}\text { Scores IPR Similarity, TS, Results Mix in } \\
\text { Drudge } 1\end{array}$ & 6 & 7 & 5 & 12 & 22 & 141 & 37 & 18 & 248 & 248 \\
\hline in percent of the overall scores & $0.4 \%$ & $0.5 \%$ & $0.4 \%$ & $0.9 \%$ & $2 \%$ & $10 \%$ & $3 \%$ & $1.3 \%$ & $0 \%$ & $18 \%$ \\
\hline $\begin{array}{l}\text { Scores IPR Similarity, TS, Results Mix in } \\
\text { Drudge } 2\end{array}$ & 5 & 1 & 6 & 1 & 62 & 78 & 56 & 9 & 218 & 218 \\
\hline in percent of the overall scores & $0.4 \%$ & $0.1 \%$ & $0.4 \%$ & $0.1 \%$ & $5 \%$ & $6 \%$ & $4 \%$ & $0.7 \%$ & $0 \%$ & $16 \%$ \\
\hline $\begin{array}{l}\text { Overall scores IPR Similarity, TS, Results } \\
\text { Mix, both Drudges }\end{array}$ & 9 & 8 & 9 & 13 & 84 & 202 & 92 & 27 & 444 & 444 \\
\hline in percent & $0.7 \%$ & $0.6 \%$ & $0.7 \%$ & $1 \%$ & $6 \%$ & $15 \%$ & $7 \%$ & $2 \%$ & $33 \%$ & $33 \%$ \\
\hline
\end{tabular}

and Results Mix of both Drudges (between 17 and $26 \%$ ). In the TS this vegetation type is less significant $(7 \%)$. Most of the scores are allocated to the early Eocene to early Oligocene sites of Hampshire Basin to Rauenberg (Figure 5). IPR Similarity and Results Mix of both Drudges also indicate a relationship to "BLEF China, Japan" for the early to early late Miocene Central European sites of Linz, Oberdorf, Wackersdorf, Arjuzanx, and Mataschen, but to a far lesser and decreasing extent (Figure 2). To a minor extent, this vegetation type is also indicated for the Pliocene of South Europe, especially for Ca'Viettone. In the TS, "BLEF China, Japan" is relevant only for some of the early Eocene sites (Figure 3).

At the level of vegetation units, China 36 received the highest percentage $(>2 \%)$, followed by China $38(2 \%)$, China 34 and China 17 (almost $2 \%$ ), and China 32, 33, 35, and Japan 04 (at least $1 \%$ ) of the overall scores (Appendix 8, sheet "BLEF China, Japan"). China 01 appears only for the early Eocene sites of Hampshire Basin and London
Clay. China 16 to 22, 30, 33, 34, and Japan 07-14 are relevant for the early Eocene to early Oligocene, whereas China 35, 37, and Japan 04 appear more often as proxies for early Oligocene to early Miocene sites (Seifhennersdorf, Linz, Oberdorf, Wackersdorf) than for Eocene ones (Appendix 9, sheet "BLEF China, Japan").

"MMF China". This type includes seven units, of which $86 \%$ (6) appear as proxies for the fossil plant assemblages (Table 4). Eleven percent of the overall scores are allotted with this vegetation type. "MMF China" is especially relevant in the TS $(20 \%)$ and the Results Mix of Drudge $2(18 \%)$. In contrast, it is of minor relevance in the IPR Similarities of both Drudges (2-4\%) (Table 4, Figures 2-4). In the IPR Similarity of Drudge 1, "MMF China" is indicated for Pliocene sites in Central Europe (Sessenheim to Kranichfeld), while its occurrence appears scattered in the IPR Similarity derived by Drudge 2 (Figure 2). Seifhennersdorf received the highest number of scores for "MMF China" (almost $50 \%$, 


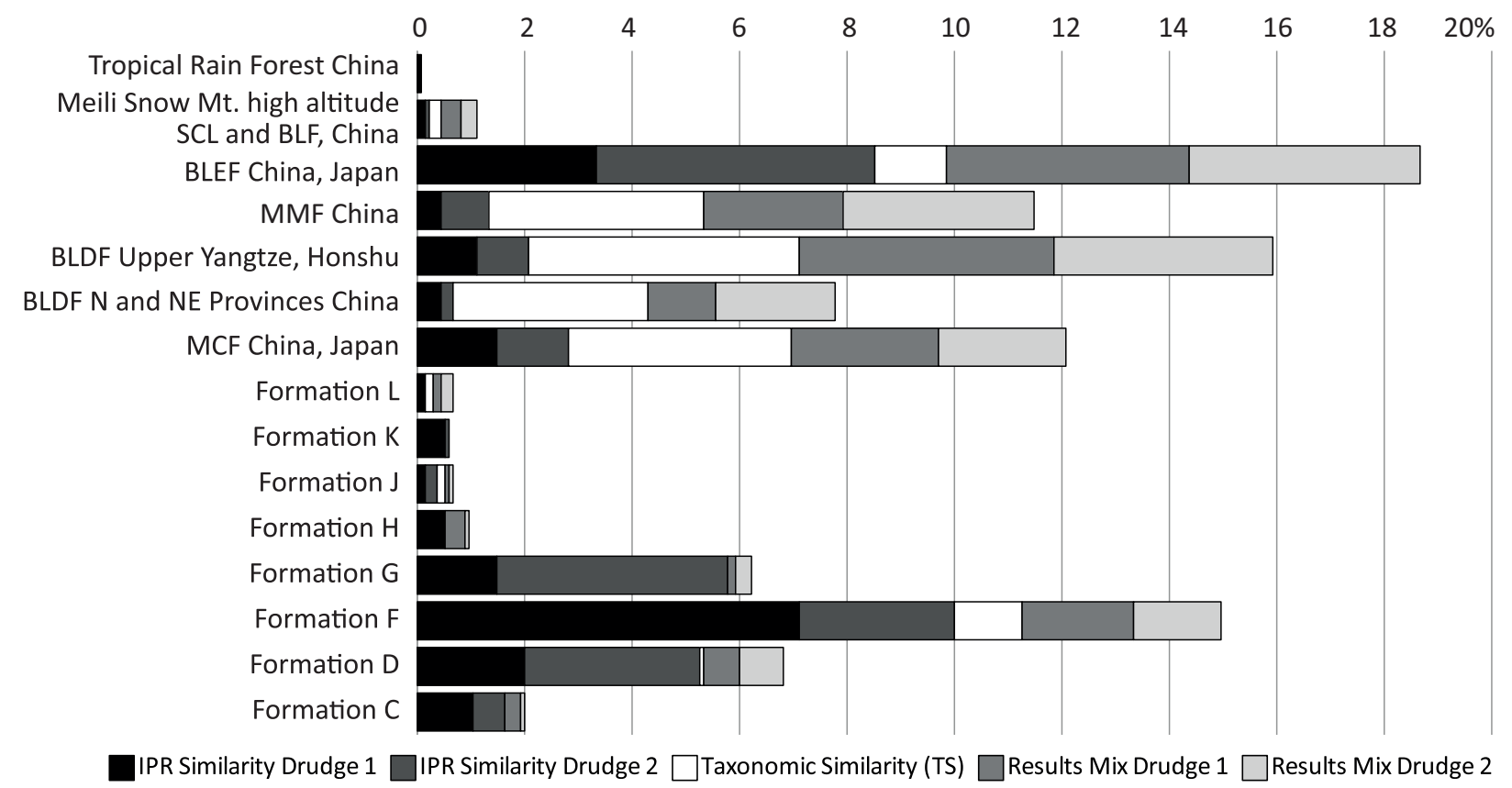

FIGURE 4. Representation of East Asian and European vegetation types and formations as delivered by Drudges 1 and Drudge 2 for the IPR Similarity, Taxonomic Similarity (TS), and Results Mix. See also Appendix 8.

12 of 25 scores) from the whole set of fossil sites (Figure 5, Appendix 9, sheet "MMF China").

Regarding individual units, China 57 and China 60 received more than $3 \%$ of the overall scores, followed by China 61 (> 2\%) (Appendix 8, sheet "MMF China").

"BLDF Upper Yangtze, Honshu". This type comprises 17 units, of which 11 (65\%) represent proxies for the fossil assemblages. Sixteen percent of all scores are allotted to this vegetation type (Table 4). "BLDF Upper Yangtze, Honshu" is delivered mainly by the TS $(25 \%)$ and the Results Mix of both Drudges (24\% by Drudge 1 and $20 \%$ by Drudge 2 ), whereas its proxies are subordinate and scattered in the IPR Similarities $(6 \%$ by Drudge $1,5 \%$ by Drudge 2; Table 4, Figures 2-4). In the overall score, "BLDF Upper Yangtze, Honshu" is less relevant in the Eocene to early Oligocene and in the early Pleistocene of Armenia than in the Miocene to Pliocene of all over Europe and Turkey (Figures $3,5)$. Vegetation units from China are mainly restricted to the period covered by the sites from Seifhennersdorf to the oldest assemblage from Armenia (Shamb 1b+A1) (Appendix 9, sheet "BLDF Upper Yangtze, Honshu").

On the level of units, Japan 05 and China 67 achieved more than $4 \%$ of the overall scores, followed by Japan 01 (> 3\%), and China 63 (nearly 2\%) (Appendix 8, sheet "BLDF Upper Yangtze, Honshu").
"BLDF N and NE Provinces, China". From this type, which is split into 12 units, five units (42\%) achieved almost $8 \%$ of the overall scores (Table 4 , Figure 4). In the TS it achieved $18 \%$ of the scores, followed by the Results Mix of Drudge $2(11 \%)$ and that of Drudge $1(6 \%)$. In the IPR Similarities this vegetation type is least relevant (2\% IPR Similarity of Drudge 1,1\% in that of Drudge 2). For the Eocene to early Miocene, "BLDF N and NE Provinces, China" is least relevant (Figure 3 Overall score, Figure 5). Otherwise this vegetation type occurs in the proxy sets mainly from the Miocene onwards. The highest number of scores were received by the assemblages from the Plio-/Pleistocene of Rippersroda and Klinger beds and the early Pleistocene assemblages from Armenia (Figure 5).

China 68 achieved by far the highest number of the overall scores (nearly $5 \%$ ), followed by China 71 (almost 2\%) (Appendix 8, sheet "BLDF N, NE Provinces, China"). The other units received less than $1 \%$ in the overall scores.

"MCF China, Japan". This type comprises 18 units of which $14(78 \%)$ appear as proxies derived by the Drudges (Table 4). Twelve percent of the overall scores are allocated with this vegetation type. The maximum score was allocated from TS (21\%), followed by that of the Results Mix derived by Drudge $1(14 \%)$ and that by Drudge $2(12 \%)$. In the IPR Similarities of both Drudges the scores 
Meili Snow Mt.

high altitude

SCL and BLF,

China

BLEF
China, Japan China

BLDF

Upper Yangtze, N, NE Provin- MCF

$\begin{array}{llll}0 & 5 & 10 & 15\end{array}$

Hampshire Basin

London Clay

Messel

Geiseltal

Profen-Scheiplitz

Staré Sedlo

Flörsheim

Rauenberg

Seifhennersdorf

Linz, Ebelsberg Fm.

Oberdorf

Mecsek Mts.

Wackersdorf

Horní Bříza

Randeck Maar

Parschlug

Wieliczka

Gdów Bay

Staré Gliwice

Holy Cross Mts.

Erdöbenye

Arjuzanx

Mataschen

Lohnsburg

Poštorná-Moravská N.V.

Sośnica

Ruszów

Sessenheim

Auenheim

Tachov Graben

Kaltensundheim

Vildštejn Fm., Pluto Clay

Vildštejn Fm., Nero Clay

Gerstungen

Kranichfeld

Berga

Vildštejn Fm., lignite beds

Rippersroda

Vildštejn Fm., upper beds

Nordhausen

Klinger beds

Güvem

Kymi, Euboea

Yatagan Basin

Pitsidia, Crete

Mt. Tondo

Tossignano

Ca'Viettone

Shamb $1 b+A 1$

Shamb $1 f+B 1$

Darbas $2 / d$

Uyts-2/a + Uts-2 PZ23

Darbas-2/f + Drb-2 PZ6

Tolors 2

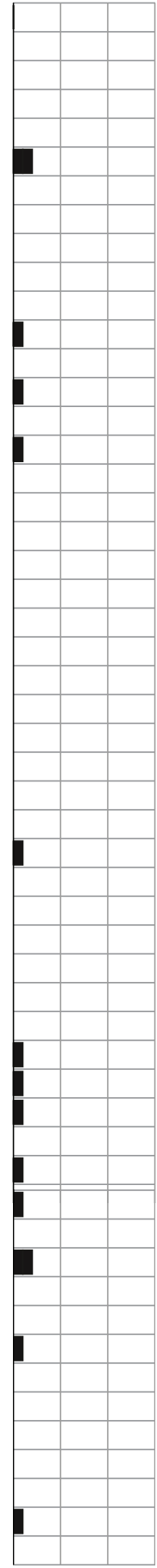

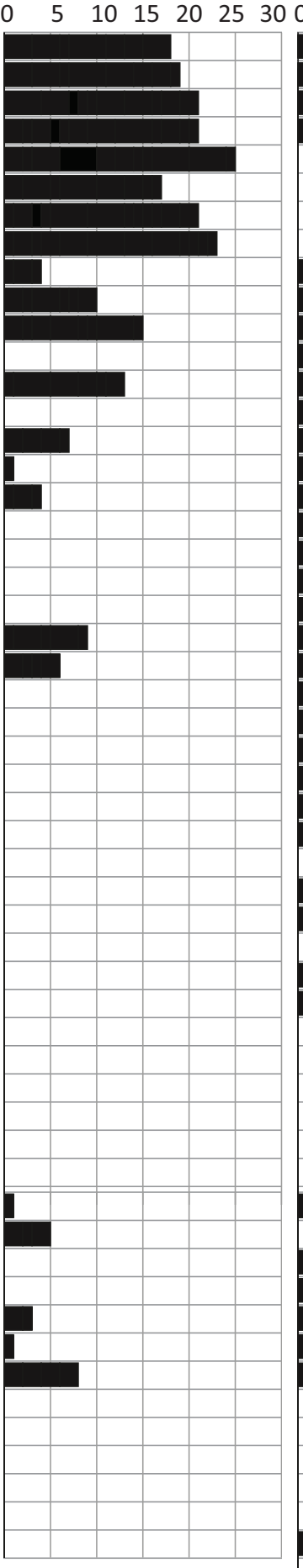

Honshu ces, China China, Japan

$\begin{array}{llllllllllllllll}0 & 5 & 10 & 15 & 0 & 5 & 10 & 15 & 0 & 5 & 10 & 15 & 0 & 5 & 10 & 15\end{array}$
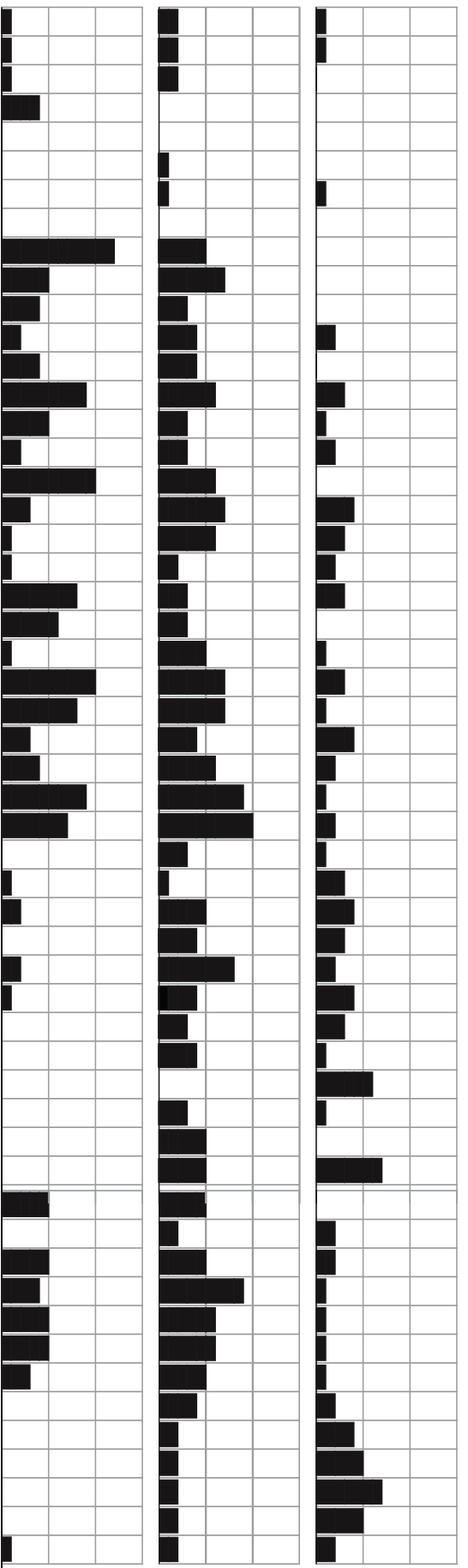
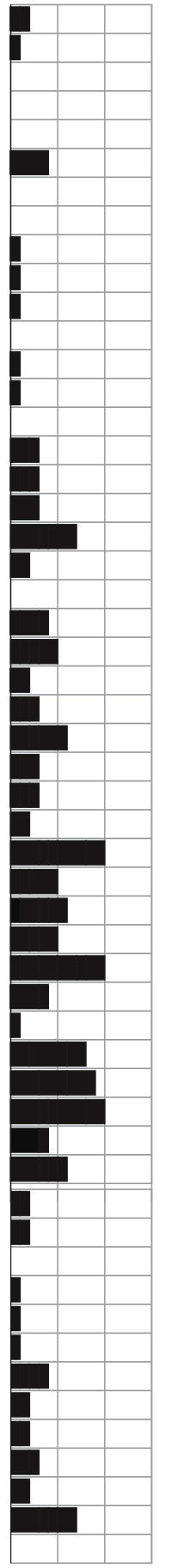

FIGURE 5. Representation of modern East Asian vegetation types for the test set of fossil assemblages as delivered by Drudges 1 and 2. More detailed information on units is available in Appendix 9. 
amount to $7 \%$ (Table 4, Figure 4). Proxies of "MCF China, Japan" appear only very sporadically from the Eocene to the late early Miocene in Central Europe (Figure 5) but then the relevance of this vegetation type increases during the Miocene and is highest in the Plio-/Pleistocene of Central Europe. Units Mongolia 1 and 2 are restricted to assemblages of the Central European Plio-/Pleistocene (Appendix 9, sheet "MCF China, Japan").

Japan 06 and China 81 achieved $3.5 \%$ of the overall counts, followed by Mongolia 1 (almost 2\%) (Appendix 8, sheet "MCF China, Japan"). All other units received only up to $0.5 \%$ of the overall scores.

\section{European Vegetation Formations}

European vegetation formations are delivered as proxies mainly by the IPR Similarities of both Drudges and to a minor extent by the Results Mix of both Drudges (Figures 2, 4). In the TS the scores are almost entirely restricted to the Plio-/ Pleistocene of Central Europe and to a minor extent to the early Pleistocene assemblages of Armenia (Figure 3). Due to the higher numbers of units in the European Formations $C$ to $G$ compared to East Asian vegetation types, the scores appear more scattered and their numbers per unit are usually lower for the European vegetation formations.

Formations $\mathrm{L}, \mathrm{K}, \mathrm{J}$, and $\mathrm{H}$ achieved between $0.6 \%$ and $1 \%$ of the overall scores (Table 4 , Figure 4). Formation $L$ is largely restricted to the Pleistocene of Armenia (Figure 3, Overall score). For Formation $\mathrm{K}$ the scores are restricted to the IPR Similarity mainly of Drudge 1 , to the middle Miocene of Central Europe (Randeck Maar, Parschlug, Holy Cross Mts.) and to the Miocene of Southern Europe (Kymi, Mt. Tondo, Tossignano) (Figure 2). Proxies of vegetation Formation $\mathrm{H}$ are scattered in the IPR Similarity of Drudge 1 and in the Results Mix of both Drudges (Figures 2, 6). Sporadically, $\mathrm{H} 001$ is delivered for assemblages from the Miocene in Central Europe and the Pliocene in the Mediterranean, with higher proportions of broadleaved evergreen taxa (Linz, Ebelsberg Fm., Oberdorf, Wackersdorf, Mataschen), while $\mathrm{HOO2}$ is delivered for some early to middle Miocene assemblages and occasionally for the Mediterranean Miocene and the Armenian Pleistocene; $\mathrm{HOO}$ is restricted to the late Miocene site Poštorná-Moravská N.V. (Figure 7, Appendix 9, sheet "Formation H").

Formations $G, F$, and $D$ received more than $5 \%$ each of the overall scores (Table 4, Figure 4) and therefore are treated here in more detail.
Vegetation Formation G. Formation G, consisting of 77 units, received $6 \%$ of the overall scores. The scores went to 35 of its units (45\%) (Table 4). The scores are mainly allocated with the IPR Similarities $(7 \%$ of the scores in Drudge $1,21 \%$ in Drudge 2, Figure 4). In the IPR Similarity of Drudge 1, Formation $G$ is more relevant for the record from the Mediterranean and Armenia than for other regions (Figure 2, Appendix 4, sheet "IPR Similarity Drudge 1 graph"). In the IPR Similarity of Drudge 2 it is relevant for Central European assemblages, mostly from the late early/middle Miocene (Parschlug to Erdöbénye) and for South Europe and Armenia (Figure 2, Appendix 4, sheet "IPR Similarity Drudge 2 graph"). In the overall score, Formation $\mathrm{G}$ is most relevant for the early Pleistocene of Armenia, followed by the late early to middle Miocene of Central Europe and the Mediterranean Miocene. It is not indicated for the Eocene to the early Miocene and is of low relevance for the late Miocene and Plio-/Pleistocene of Central Europe (Figures 3, 6, Appendix 4, sheet "Overall proxy graph").

Regarding its four subdivisions, G.2 is most relevant (Figure 7, Appendix 9, sheet "Formation G"), and delivered mainly for the late early/middle Miocene of Central Europe and Turkey, and the Armenian Pleistocene. It is followed by G.3, which appears as a proxy not only for the aforementioned regions but also sporadically for the (late) Pliocene taphocoenoses in Central Europe.

On the level of individual units, G036 (subdivision G.2) received the highest percentage of the overall scores $(0.5 \%)$ followed by $\mathrm{G} 017, \mathrm{G} 018$ (part of G.1), G032 (G.2), G056 (G.3), and G071 (G.4) with $0.4 \%$ each (Appendix 8, sheet "Formation G").

Vegetation Formation F. This formation, split into 172 units, received the highest number of the overall scores $(15 \%, 202)$ among the European vegetation formations (Table 4, Figure 4). The scores went to $45 \%$ (77) of its units. In the IPR Similarity of Drudge 1 this formation is represented by $36 \%$, in Drudge 2 by $14 \%$. The percentages for the TS and Results Mix of both Drudges are lower, ranging between 6\% (TS) and 10\% (Results Mix Drudge 1). Proxies of Formation $\mathrm{F}$ are not delivered for the Eocene but are increasingly relevant in the Miocene and most distinct in the Central European Pliocene. In South Europe, Turkey, and Armenia, Formation $\mathrm{F}$ is delivered more or less regularly to some extent (Figures 3, 6, Appendix 4, sheet "Overall proxy graph"). 
Formation $\mathrm{H} \quad$ Formation $\mathrm{G} \quad$ Formation $\mathrm{F} \quad$ Formation $\mathrm{D} \quad$ Formation $\mathrm{C}$

0246810024468100246681012141618200244681002446810

\begin{tabular}{|l|}
\hline Hampshire Basin \\
\hline London Clay \\
\hline Messel \\
\hline Geiseltal \\
\hline Profen-Scheiplitz \\
\hline Staré Sedlo \\
\hline Flörsheim \\
\hline Rauenberg \\
\hline Seifhennersdorf \\
\hline Linz, Ebelsberg Fm. \\
\hline Oberdorf \\
\hline Mecsek Mts. \\
\hline Wackersdorf \\
\hline Horní Bŕíza \\
\hline Randeck Maar \\
\hline Parschlug \\
\hline Wieliczka \\
\hline Gdów Bay \\
\hline Staré Gliwice \\
\hline Holy Cross Mts. \\
\hline Erdöbenye \\
\hline Arjuzanx \\
\hline Mataschen \\
\hline Lohnsburg \\
\hline Poštorná-Moravská N.V. \\
\hline Sośnica \\
\hline Ruszów \\
\hline Sessenheim \\
\hline Auenheim \\
\hline Tachov Graben \\
\hline Kaltensundheim \\
\hline Vildštejn Fm., Pluto Clay \\
\hline Vildštejn Fm., Nero Clay \\
\hline Gerstungen \\
\hline Kranichfeld \\
\hline Berga \\
\hline Vildštejn Fm., lignite beds \\
\hline Rippersroda \\
\hline Vildštejn Fm., upper beds \\
\hline Nordhausen \\
\hline Klinger beds \\
\hline Güvem \\
\hline Kymi, Euboea \\
\hline Yatagan Basin \\
\hline Pitsidia, Crete \\
\hline Mt. Tondo \\
\hline Tossignano \\
\hline Ca'Viettone \\
\hline Shamb 1b+A1 \\
\hline Shamb 1f+B1 \\
\hline Darbas 2/d \\
\hline Uyts-2/a + Uts-2 PZ23 \\
\hline Darbas-2/f + Drb-2 PZ6 \\
\hline Tolors 2 \\
\hline \\
\hline
\end{tabular}
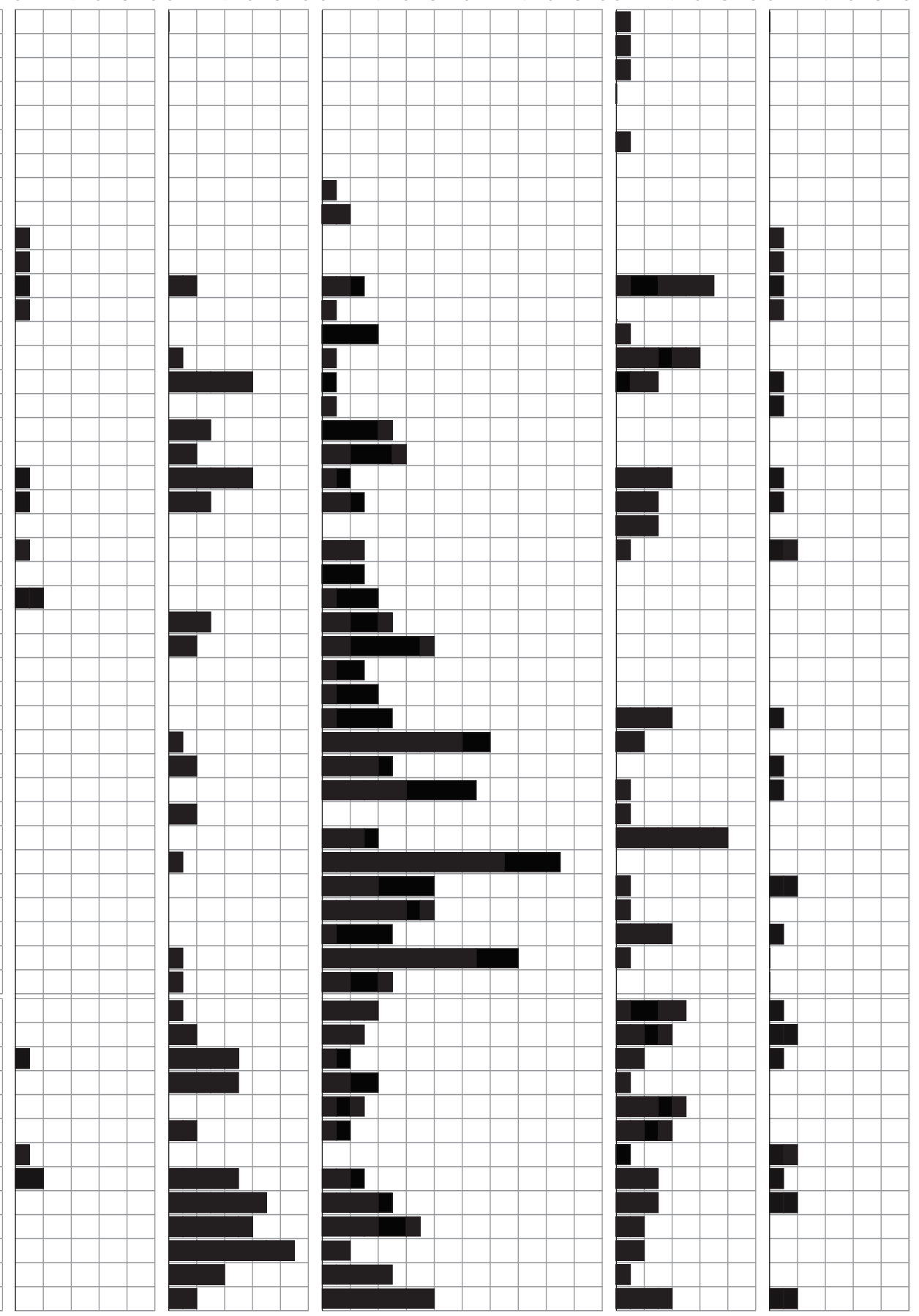

FIGURE 6. Representation of modern European vegetation formations for the test set of fossil assemblages as delivered by Drudges 1 and 2. Formation $\mathrm{H}$ - Hygrophilous thermophytic mixed deciduous broadleaved forests; Formation G - Thermophilous mixed deciduous broadleaved forests; Formation F - Mesophytic broadleaved deciduous and mixed broadleaved/conifer forests; Formation D - Mesophytic and hygromesophytic coniferous and mixed broadleaved-coniferous forests; Formation C - Subarctic, boreal and nemoral-montane open woodlands as well as subalpine and oro-Mediterranean vegetation. More detailed information on subdivisions and units is available in Appendix 9. 


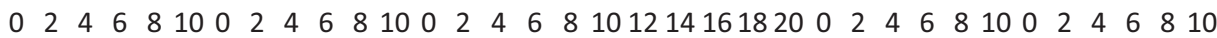

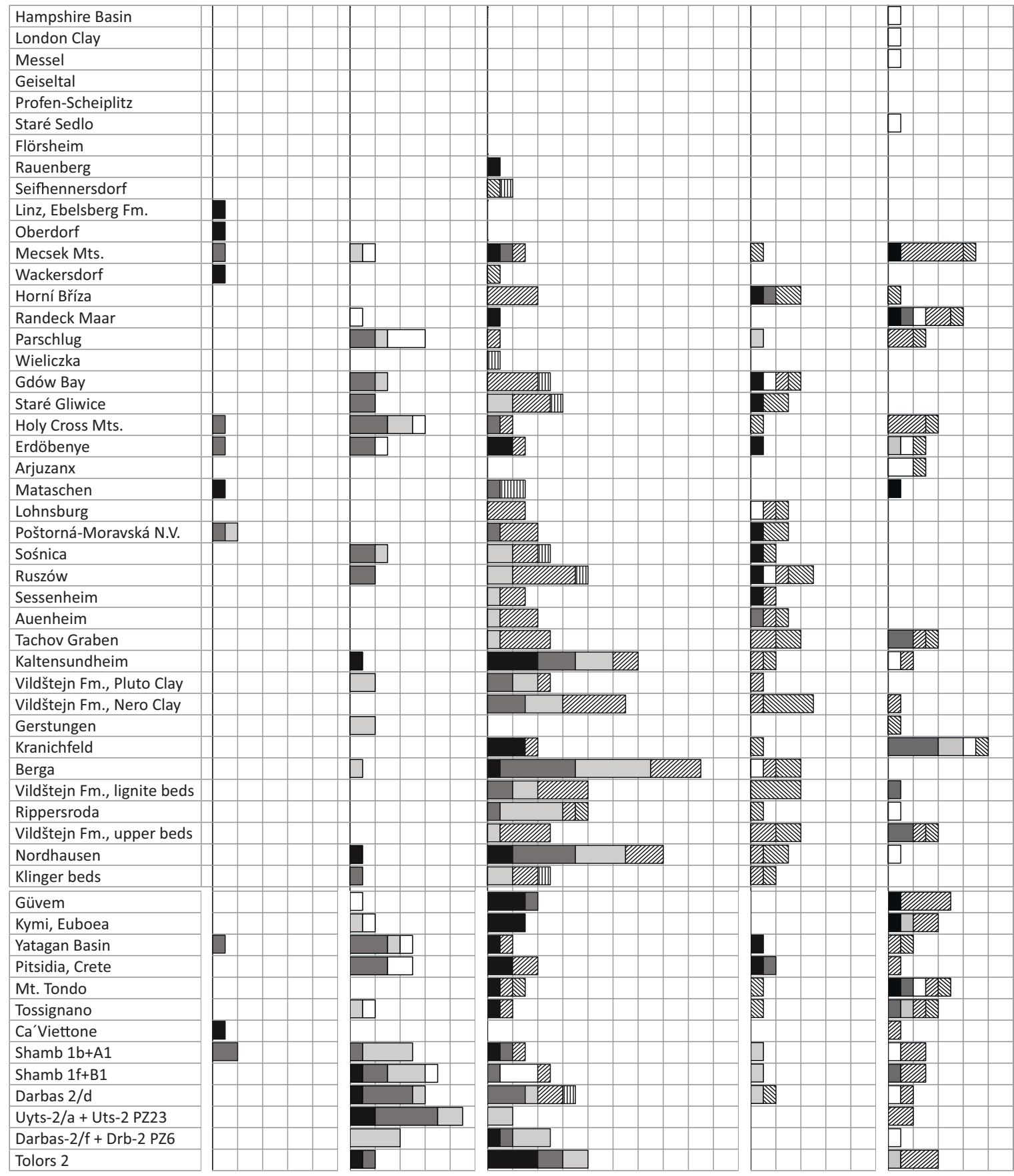

FIGURE 7 (caption on next page). 
On the level of vegetation subdivisions F.1 to F.7 (Bohn et al., 2004), F.5 received the highest percentage of the overall scores within Formation $\mathrm{F}(>5 \%)$, almost exclusively in the IPR Similarities of both Drudges. F.5 is followed by F. 3 and F.2 with around $3 \%$ each (Appendix 8, sheet "Formation F"). Forest types F.3 and F.5 are delivered rather regularly, except for F.3, which does not appear as a proxy for the Miocene record of the Mediterranean region (Figure 7, Appendix 9 sheet "Formation F"). F.2 is delivered mainly for the Pliocene of Central Europe and in the Armenian early Pleistocene. Focussing on F.5, which itself comprises 85 units in seven forest types (F.5.1.1 to 5.2.4), almost $3 \%$ went to 5.2 .3 and 4 (Figure 7, Appendix 8, sheet "Formation F", Appendix 9, "sheet Formation F").

Focussing on individual units, F033 received by far the highest percentage of the overall scores (1.5\%), followed by F043 and F075 (0.7\% each) (Appendix 8, sheet "Formation F").

Vegetation Formation D. Formation D (64 units) received $7 \%$ of the overall scores (Table 4 ). This value is very close to that of Formation G. The scores are allotted to 26 of its units $(41 \%)$. As in Formations $\mathrm{G}$ and $\mathrm{F}$, Formation $\mathrm{D}$ received more scores in the IPR Similarities (10\% Drudge 1, 16\% Drudge 2$)$ than by the TS $(0.4 \%)$ and by the Results Mix (3\% Drudge 1, 4\% Drudge 2) (Table 4,
Figure 4). This formation is relevant for the late early Miocene, the Plio-/Pleistocene of Central Europe, and the record from the Mediterranean and Armenia (Figures 3, 6, Appendix 4, sheet "Overall proxy graph").

Regarding the subdivisions D.1 to D.6, D.5 received the highest number of scores (nearly $3 \%$ ) (Appendix 8, sheet "Formation D"). This forest type includes the units D052 and D042, which received the highest percentages of the overall scores in this formation (D052 1\%, D042 0.7\%). Unit D064, which is part of D.6, also received $0.7 \%$ of the overall scores.

Proxies of the forest types D.4 and D.5 are mainly delivered for some early to middle Miocene Central European sites (Figure 7, Appendix 9, sheet "Formation D"). For the Mediterranean and Armenian record, the subdivisions D.1 to D.6 appear to some extent, but only forest type D.5 is a regularly appearing proxy. In the Plio-/Pleistocene, forest type D.2 is important for Central Europe.

Vegetation Formation C. Formation C (47 units) received only $2 \%$ of the overall scores (Table 4 ). The scores, which are allocated with $28 \%$ (13) of its 47 units, occur mainly scattered in the IPR Similarities (5\% Drudge 1, 3\% Drudge 2 of the scores there) (Figure 2, Table 4). Stratigraphically the proxies are scattered over the Miocene and Pliocene (Figure 6). Out of the 3 subdivisions, only

FIGURE 7 (previous page). Representation of modern European vegetation formations for the test set of fossil assemblages as delivered by Drudges 1 and 2 in more detail (see also Appendix 9).

Formation $\mathrm{H}$ : H001, Colchic lowland to submontane mixed oak forests, in black; H002, Hyrcanian lowland-colline mixed broadleaved forests, in dark grey; H003, Hyrcanian colline to montane oak forests, in light grey.

Formation G: G.1 - Subcontinental thermophilous (mixed) pedunculate oak and sessile oak forests, in black; G.2 - SubMediterranean-subcontinental thermophilous bitter oak and Balkan oak and mixed forests, in dark grey; G.3 - Sub-Mediterranean and meso-supra-Mediterranean downy oak and mixed forests, in light grey; G.4 - Iberian supra- and mesoMediterranean oak forests, in white.

Formation F: F.1 - Species-poor acidophilous oak and mixed oak forests, in black; F.2 - Mixed oak-ash forests, in dark grey; F.3 - Mixed oak-hornbeam forests, in light grey; F.4 Lime-pedunculate oak forests, in white; F.5 - Beech and mixed beech forests, hatched lower left to upper right; F.6 - Oriental beech forests and hornbeam-oriental beech forests, hatched upper left to lower right; F.7 - Caucasian mixed hornbeam-oak forests, hatched vertically.

Formation F, F.5 - Beech and mixed beech forests: F.5.1.1 - Species-poor oligotrophic to mesotrophic beech and mixed beech forests, lowland(-colline) types, in black; F.5.1.2 - Species-poor oligotrophic to mesotrophic beech and mixed beech forests, colline-submontane types, in dark grey; F.5.1.3 - Species-poor oligotrophic to mesotrophic beech and mixed beech forests, montane-altimontane types, in light grey; F.5.2.1 - Species-rich eutrophic and eu-mesotrophic beech and mixed beech forests, colline-submontane types, in white; F.5.2.2 - Species-rich eutrophic and eu-mesotrophic beech and mixed beech forests, colline-submontane types, hatched lower left to upper right; F.5.2.3 and 4 - Species-rich eutrophic and eu-mesotrophic beech and mixed beech forests, montane-altimontane types, hatched upper left to lower right.

Formation D: D.1 - Western boreal spruce forests, in black; D.2 - Eastern boreal pine-spruce and fir-spruce forests, in dark grey; D.3 - Hemiboreal spruce and fir-spruce forests with broad-leaved trees, in light grey; D.4 - Montane to altimontane, partly submontane fir and spruce forests in the nemoral zone, in white; D.5 - Boreal and hemiboreal pine forests, hatched lower left to upper right; D.6 - Montane to altimontane (subalpine) pine forests in the nemoral zone; hatched upper left to lower right. 
units of C.2 and C.3 are delivered as proxies (Appendix 9, sheet Formation C). In the overall scores, the maximum is $0.4 \%$ for $\mathrm{C} 045$ followed by C046 (0.3\%) (Appendix 8, sheet "Formation C").

\section{DISCUSSION}

\section{IPR Similarities, Taxonomic Similarity (TS) and Results Mix in Comparison}

For Central Europe, the IPR Similarities of both Drudges indicate close East Asian affinities from the early Eocene to the early Miocene (Hampshire Basin to Oberdorf, Wackersdorf). These affinities are more distinct in the IPR Similarity of Drudge 2 than in that of Drudge 1 (Figure 2). Starting from the early Miocene the similarities to modern European vegetation become pronounced. This result contrasts with that of the TS, in which East Asian vegetation proxies continue to dominate from the early Eocene to the Pliocene (Figure 3 ). This supports the recent results of Teodoridis et al. (2020), which were based on a stratigraphically and regionally far less comprehensive test set of six Central European sites covering the timespan late Eocene to late Pliocene. The strong impact of the vegetation types "MMF China", "BLDF Upper Yangtze, Honshu", and "MCF China, Japan", all represented by more than $20 \%$ of the TS scores, is related to their remarkable floristic diversity. In contrast, modern European vegetation is considerably depleted as a result of the climatic stress during the Quaternary Ice Age. This fact probably influenced the TS results and accounted for the differences between the results in the IPR Similarities and TS. The European Plio-/Pleistocene assemblages of Kaltensundheim, Berga, and Nordhausen (Thuringia) show strong ties to the modern flora of Europe (Formation F), while in the early Pleistocene Armenian record, the taxonomic similarity to modern East Asia still remains stronger than to Europe (Figure 3). The Great Caucasus mountain ranges and the proximity of the Caspian Sea were potentially favourable for these ties.

\section{Vegetation Evolution Reflected by Modern Proxies}

From the early Eocene to the early Oligocene (Hampshire Basin to Rauenberg), the overall results indicate closest similarities to East Asian broad-leaved evergreen forests "BLEF China, Japan" (Figure 3); this signal is the most distinctive of all results. It is based on the highest percentages of the BLE component of the whole data set. Compared to the IPR Similarities and the Results Mix of both Drudges, this trend is less distinct in the Taxonomic Similarity (TS), where "MMF China" and "BLDF Upper Yangtze, Honshu" represent essential proxies (Figure 3).

Remarkably, early Eocene assemblages do not point towards tropical vegetation, which may support interpretations of "paratropical" rather than tropical conditions (e.g., for Hampshire Basin, London Clay, Messel; Collinson, 1983; Wilde et al., 2005). However, in our reference set of modern vegetation, representation of tropical vegetation is currently restricted to China. We cannot exclude that tropical vegetation in adjacent southern parts of Asia would be more suitable for comparison with the fossil record if they were included.

The early Oligocene assemblage of Seifhennersdorf indicates a significant turnover in overall leaf physiognomy of zonal woody angiosperms by lower percentages of the BLE component and a higher one of the BLD component (Appendix 1, sheet "IPR results all fossil sites"). Especially in the proxy set of the IPR Similarities this is reflected by a higher variability of proxies (Figure 2). Summarising the results for Seifhennersdorf derived by both Drudges (IPR Similarities, TS, and Results Mix) points to the close affinities to "MMF China" and "BLDF Upper Yangtze, Honshu" and weaker ones to "BLEF China, Japan" (Figure 3, Overall score). This signal clearly reflects the invasion of broadleaved deciduous taxa "Arctotertiary taxa" and conforms to the empirically retrieved results for this site (Walther and Kvaček, 2007).

The early Oligocene plant assemblages of Flörsheim and Rauenberg are close in age to Seifhennersdorf (see Table 1). The plant assemblage from Seifhennersdorf was deposited at the northern margin of the Palaeogene European continent in maar lake sediments, probably close to the growing site, whereas the assemblages of Flörsheim and Rauenberg accumulated in marine sediments of the Rhine graben, when plant remains from the southwestern margin of the European continent were washed into the sea. At that time, the Rhine graben formed the marine connection between the North Sea and the Paratethys Ocean in the south. Contrary to Seifhennersdorf, the overall scores for Flörsheim and Rauenberg clearly point towards "BLEF China, Japan" (Figure 3). The vegetation along the coastal regions of the Rhine graben may have been influenced by more favourable climatic conditions, allowing for the growth of highly diverse broad-leaved evergreen forests (Kvaček, 2004, Kovar-Eder, 2016). The constraints of the age correlation for Seifhennersdorf and the 
Rhine graben sites, as well as differences in the specific taphonomic processes, may, however, somewhat relativise our interpretation regarding the differences in flora, vegetation and climate.

"BLEF China, Japan" is still relevant in Central Europe in the early Miocene (Linz, Oberdorf, Wackersdorf) (Figure 5), but in the middle to late Miocene its proxies are mainly restricted to a few sites such as Arjuzanx and Mataschen, with an overall trend of decreasing relevance. In southern Europe, "BLEF China, Japan" appears to a variable extent (Kymi, Mt. Tondo, Tossignano, Ca'Viettone); except for Ca'Viettone, however, it is less pronounced than in the Palaeogene and Neogene of Central Europe. The peaks of "BLEF China, Japan" in the Neogene record reflect the so-called "Younger Mastixioid" floras (sensu Mai, 1964), which are interpreted to indicate warm and humid climatic oscillations or relict vegetation in favourable habitats. For these assemblages, modern European vegetation proxies appear to be less relevant.

For the Palaeogene, prevailing proxies of the "BLEF China, Japan" are BLEF of Longqi Mt. (China 16 to 22), Broad-leaved Evergreen Sclerophyllous Forests Taiwan, Fujian, and Guangxi (China 30, 33, 34), and BLEF on Honshu (Shiroyama, Nara) and Yakushima (Japan 07 to 14) (Appendix 9, sheet "BLEF China, Japan"). These proxies are characterised by the lowest proportions of the BLD component and the highest ones of the BLE component among all modern proxies (Appendix 6, sheet "Asia"). For the Palaeogene and Neogene, the shared proxies of "BLEF China, Japan" are mainly Broad-leaved Evergreen Sclerophyllous Forests in the Maritime Provinces Guangdong and Southern Hunan (China 32, 35), and in the Southeastern Provinces (Guizhou, Northern Guangxi) and Yunnan (China 36 to 38). For the late early and middle Miocene and Pliocene record, however, the proxies are mainly restricted to Broad-leaved Evergreen Sclerophyllous Forest Southern Hunan, the Southeastern Provinces and Yunnan (China 35 to 38 ), which are distinguished by higher percentages of the BLD component and lower ones of the BLE component (Appendix 6, sheet "Asia"). BLEF Mt. Fuji (Japan 04) is relevant for Neogene assemblages with elevated proportions of the BLE component such as Linz, Oberdorf, Wackersdorf, Wieliczka, Arjuzanx, and $\mathrm{Ca}$ 'Viettone. These results clearly indicate that the "Older" and "Younger Mastixioid" floras (sensu Mai, 1964) are distinct from each other not only based on their floristic composition but also their leaf physiognomy, and these differences are reflected by the modern proxies. The youngest site of the Central European assemblages included here with considerable relationship to the vegetation type "BLEF China, Japan" is the early late Miocene assemblage of Mataschen.

In Central Europe, "MMF China" and "BLDF Upper Yangtze, Honshu" represent relevant proxies from the middle Oligocene (Seifhennersdorf) to the Pliocene (Sessenheim, Auenheim) (Figure 5). Their high taxonomic diversity - including relict taxa with affinities to the fossil record in Europe and Asia Minor - accounts for the strong taxonomic similarity. "MMF China" (especially China 57,60 and 61 ) is most relevant for the early Oligocene (Seifhennersdorf) and the early Miocene to Pliocene of Europe (Linz, Ebelsberg Fm. to Auenheim) and the Mediterranean and Asia Minor (Güvem to Tossignano) (Appendix 9, sheet "MMF China"). China 57, 60 and 61 represent the MMF in the mountain regions of the adjacent provinces Zhejang (Tientai Shan), Anhui and Jiangxi (Lu Shan). These forests include between 73 and 113 zonal woody taxa, of which 84 to $86 \%$ are assigned to the BLD component and 14 to almost $16 \%$ to the BLE component (Appendix 6, sheet "Asia"). In the Plio-/Pleistocene of Europe, Turkey, and Armenia, "MMF China" drastically loses its relevance.

Within "BLDF Upper Yangtze, Honshu", Shirakami Sanchi - Broad-leaved Deciduous Forest, Lindera membranacea-Fagus crenata community (Japan 01) and Mt. Fuji - Broad-leaved Deciduous Forest - Fagus crenata region (Japan 05) are important proxies especially for the middle Miocene and Plio-/Pleistocene of Central Europe (Appendix 9, sheet "BLDF Upper Yangtze, Honshu"). Both are important communities of the broad-leaved deciduous forest region. Shirakami Sanchi is situated in northwestern Honshu by the Sea of Japan, which is characterised by a precipitation maximum in winter. This climate enables some low-growing evergreen taxa to survive, accounting for a slightly elevated proportion of the BLE component in the IPR vegetation analysis (Appendix 6, sheet "Asia"). Mt. Fuji is located at the Pacific side of Honshu. Both units are characterised by high numbers of zonal woody angiosperms $(>80)$ as is the Broad-leaved Deciduous Forest (Eastern Guizhou) (China 67). This latter unit which is an important proxy for the European and Turkish Miocene and the Mediterranean Pliocene is part of the Deciduous Broad-leaved Forest of the Upper Yangtze Provinces (sensu Wang, 1961). It 
includes 15 conifer species and relict taxa as Cunninghamia and Ginkgo.

In the Plio-/Pleistocene, "BLDF $\mathrm{N}$ and NE Provinces" and "MCF China, Japan" gain increasing importance as proxies, whereas the relevance of "BLDF of the Upper Yangtze, Honshu" decreases (Figures 3,5). This trend is mainly based on closer taxonomic relationship on the genus level to "BLDF $\mathrm{N}$ and NE Provinces" and "MCF China, Japan" than to "BLDF of the Upper Yangtze, Honshu". Within "MCF China, Japan" Montane Coniferous Forest - Picea obovata-Abies sibirica Forest (Mongolia 1) and Montane Coniferous Forest - Mixed Hardwoods (Mongolia 2) are delivered proxies only for the Plio-/Pleistocene of Central Europe (Appendix 9, sheet "MCF China, Japan").

In the late Miocene to early Pliocene of Central Europe (Lohnsburg to Auenheim), the overall affinities to modern vegetation of East Asia is higher than that to modern European vegetation. In the later Plio-/Pleistocene (assemblages of the Vildštejn Fm. and from Thuringia), proxies of European vegetation gain increasing relevance (Figure 3 ; see next section).

The fossil record of South Europe, Turkey, and Armenia is far less continuous than that from Central Europe, making general trends difficult to assess. For South Europe the composition of the overall scores is comparable to those of some middle Miocene sites in Central Europe, including the European Formations G, H, J, and K (Figure 3). East Asian affinity is higher in the Mio-/Pliocene record of South Europe and Turkey than in the Armenian early Pleistocene. For Ca'Viettone, "BLEF China, Japan" is the most relevant among all assemblages from the Mediterranean. The composition of its overall score is reminiscent of some Central European assemblages of the Younger Mastixioid type, e.g., Wackersdorf.

\section{European Vegetation Formations}

European vegetation proxies are delivered increasingly from the early Miocene onwards. Except for the floras with higher proportions of the BLE component (some of them assigned to the "Younger Mastixioid floras", sensu Mai, 1964), the late early and middle Miocene Central European assemblages (Mecsek Mts., Randeck Maar, Parschlug, Holy Cross Mts., Erdöbénye) are distinguished by distinctly increased numbers of European vegetation proxies. These sporadically also include vegetation Formations $\mathrm{J}$ - Mediterranean sclerophyllous forests and scrub, $\mathrm{K}$ - Xero- phytic coniferous forests, coniferous woodland and scrub, and even L - Forest steppes and dry grasslands alternating with dry scrub (Holy Cross Mts.). This pattern is very different from preceding and later periods and is related to physiognomic changes reflected in the IPR similarities. It supports the results of Kovar-Eder and Teodoridis (2018) for the late early to middle Miocene floras of the wider Paratethys surroundings. Those authors concluded climatic conditions had increased seasonality in precipitation compared to earlier and later periods (see also section Modern Vegetation Proxies Reflecting Climate Change).

Formation $\mathrm{H}$ - Hygrophilous thermophytic mixed deciduous broadleaved forests, occur in the Caucasus region. Unit H001, Colchic mixed oak forests, appears as a proxy for Miocene Central European assemblages, with higher proportions of evergreen taxa (Linz, Ebelsberg Fm., Oberdorf, Wackersdorf, Mataschen), and for the Pliocene of Italy (Ca'Viettone) (Figure 7). Hyrcanian forests (H002, H003) are delivered as proxies for early to middle Miocene Central European assemblages (Mecsek Mts., Holy Cross Mts., Erdöbénye), for Poštorná-Moravská Nová Ves (late Miocene), and for the middle Miocene of Turkey (Yatagan Basin) as well as occasionally for the Armenian early Pleistocene. The Colchic mixed oak forests ( $\mathrm{H} 001)$ are developed at the eastern margin of the Black Sea, while the Hyrcanian lowland to montane forests (H002, H003) are spread at the southern margin of the Caspian Sea. In both regions the climate is evenly warm-temperate, but in the Colchic region it is perhumid with high precipitation, whereas the Hyrcanian region differs by seasonal precipitation (dry summer, precipitation peak in fall). The forests of Formation $\mathrm{H}$ are characterised by high species diversity as well as by relict and endemic taxa. They are tall-growing with a mostly two-leveled tree layer formed by broad-leaved deciduous oaks, elms, and limes. The shrub layer is diverse and often forms thickets. Due to the different climatic situations, Colchic forests include more evergreen taxa in the understorey than Hyrcanian ones, e.g., Prunus laurocerasus, Rhododendron ponticum, and others (Bohn et al., 2004). The leaf-physiognomic differentiation of the fossil assemblages, which is expressed by the IPR Similarity, is well reflected in the differentiation of proxies of vegetation Formation $\mathrm{H}$. The Colchic and the Caspian forests are usually regarded as relict forests of former vegetation. Surprisingly, they are not delivered as proxies by the TS. The floristic similarity of Formation $\mathrm{H}$ to the fossil record is therefore 
clearly less than that of East Asian vegetation types.

Formation G - Thermophilous mixed deciduous broadleaved forests, delivered proxies mainly for the late early to middle Miocene of Central Europe and even more so for the Mediterranean region and the Armenian early Pleistocene (Figures 3,6$)$. Geographically, forests of Formation $G$ are developed between Formation $\mathrm{F}$ in the north, (forest) steppes in the southeast, and Mediterranean evergreen sclerophyllous forests in the south (Formation J). Its widest extension and highest diversity of types are developed in the Pannonian Basin and on the Balkan Peninsula. Deciduous to evergreen, drought-resistant taxa (mainly oaks) prevail in the tree layer, and drought-tolerant taxa characterise the diverse herb layer (Bohn et al. 2004, EuroVegMap 2.06, map 13).

Proxies of the subdivisions G.2 to G.4 are relevant for the Central European middle Miocene and mainly for the Miocene record of southern Europe and Turkey, whereas G.1 to G.3 appear to be more relevant for the early Pleistocene record from Armenia (Figure 7).

Subdivision G.2 - Sub-Mediterranean-subcontinental thermophilous bitter oak and Balkan oak forests are characterised by easterly bound subMediterranean oaks such as Quercus cerris. Such forests are distributed in Italy and southeastern Europe to Turkey (Bohn et al., 2004, map 13). Units G032, Thracian downy oak-bitter oak forests, and G036, Thracian mixed Balkan oak-bitter oakgrey oak forests, which occur in the Danubian and Eastern Balkan region, are the most important proxies. In both, the tree layer is two- to three-storied, and in both, the understorey and herb layer are diverse (Bohn et al., 2004).

Subdivision G.3 - Sub-Mediterranean and meso-supra-Mediterranean downy oak forests as well as mixed forests, are thermophilous forests that are well distributed in more southern regions of Europe up to the foothills of the Caucasus under warm, winter-moist and summer-dry climate (see Bohn et al., 2004, EuroVegMap 2.06, map 13). The relevant proxy G056, Balkanic-Rhodopean Oriental hornbeam-hop-hornbeam forests, are part of G.3.3 - Italian-Balkan colline to montane hop hornbeamdowny oak forests and mixed hop hornbeam forests. These two- to three-layered, low- to mediumgrowing forests have a diverse herb layer characterised by thermophilous species.

From the late early Miocene onwards, proxies of vegetation Formation F - Mesophytic broadleaved deciduous and mixed broadleaved/conifer forests are delivered for the Central European record (Figures 3,6). Its peaks are bound to the Plio-/Pleistocene of Central Europe (Vildštejn Fm., assemblages from Thuringia). Formation $F$ represents the vegetation in more or less oceanic European regions of the temperate zone (Bohn et al. 2004, EuroVegMap 2.06, map 9). It is characterised by few deciduous tree species, namely pedunculate oak, hornbeam, lime, maples, ash, and elms. European beech is limited to the west and to submontane regions, while sessile oak and field maple are bound to the east. Proxies of F.5 - Beech and mixed beech forests, received the highest score number (Figure 7) and, within these forests, species-rich eutrophic and eu-mesotrophic beech and mixed beech colline to altimontane forests (F.5.2.2 to F.5.2.4), in which the tree layer is dominated by beech (Appendix 9, sheet "Formation F").

For the Plio-/Pleistocene of Central Europe, F.3 - Mixed oak-hornbeam forests are relevant proxies (Figure 7, Appendix 9, sheet "Formation F"). Forests of F.3 appear in lowland to lower montane temperate to submeridional regions of Central and southeastern Europe. The tree layer is two- to four-storied. Compared to beech forests, the tree layer is more open, allowing for a well-developed herb layer.

Within F.2 - Mixed oak-ash forests, F033, Cantabrian-Euscaldian mixed oak-ash forests, which are relevant for the Central European Pliocene and the Armenian Pleistocene, received by far the highest score number within Formation $F$. No other European vegetation unit received such a value in TS. Three-fourths of these scores are associated with the Central European Plio-/Pleistocene record in TS and the Results Mix of both Drudges (Appendix 8, sheet "Formation F") indicating floristic relationship between the Plio-/Pleistocene Central European record and this forest type. The Cantabrian-Euscaldian mixed oak-ash forests are restricted to the western Pyrenees along the Bay of Biscay and the Cantabrian Mountains. They develop under a humid-warm climate. The tree layer is dominated mainly by pedunculate oak and European ash associated with field maple, chestnut, elms, lime and others. Characteristic are (sub)mediterranean and endemic species (Bohn et al., 2004).

In the late early to middle Miocene and Pliocene Central European record as well as the Mediterranean and Armenia, Formation D - Mesophytic and hygromesophytic coniferous and mixed broadleaved-coniferous forests, is relevant (Figures 3, 6). D.5 - Boreal and hemiboreal pine forests, deliv- 
ered the highest number of proxies (especially unit D052 followed by D042) and unit D064 which is part of D.6 - Montane to altimontane (subalpine) pine forests in the nemoral zone (Appendix 8, sheet "Formation D"). Unit D052, South Scandinavian pine forests on carbonate rocks, develop on shallow and dry soil. In the open tree layer, scots pine (Pinus sylvestris) is dominant. The droughtresistant understorey includes thermophilous woody elements such as juniper, Cotoneaster or Sorbus (Bohn et al., 2004). Unit D042, North Scandinavian open pine forests, is also dominated by scots pine in the tree layer but is distinguished from the southern type by the occurrence of Ericaceae bog species (Bohn et al., 2004). D064, Caucasian pine forests, occurring at higher altitudes on extremely dry, shallow or steep sites, are characterised by Pinus kochiana accompanied by birch, spruce, fir, beech, and different oak species. The shrub layer is polymorphous, depending on the site conditions, including typical Caucasian and boreal species (Bohn et al., 2004).

The relevance of Formation C - Subarctic, boreal, and nemoral-montane open woodlands as well as subalpine and oro-Mediterranean vegetation is low because its proxies appear scattered through the Miocene and Pliocene, hindering a more definitive interpretation (Figures 3, 6).

Proxies of vegetation Formation $L$ - Forest steppes (Meadow steppes alternating with nemoral deciduous forests) and dry grasslands alternating with dry scrub are generally delivered rarely as proxies for the test set of fossil assemblages. Its records are largely limited to the early Pleistocene of Armenia (Figure 3).

In the context of vegetation evolution during the Palaeogene and Neogene, Kovar-Eder et al. (2013) demonstrated, that the proportions of fruit dispersal syndromes (fleshy zoochorous, nonfleshy zoochorous, and anemochorus fruits) were correlated to forest types. In broad-leaved evergreen forests, the values of fleshy zoochorous taxa are high whereas anemochorous ones show low values. However, in broad-leaved deciduous forests the values of these traits are inverted. Modern forests in China and Japan show consistent trends (Knörr et al., 2012). Floristic composition evaluated here by the Taxonomic Similarity (TS), leaf physiognomy as assessed by the IPR vegetation analysis, and fruit dispersal syndromes are traits, which may serve as indicators of major ecosystem changes related to climate change.

\section{Modern Vegetation Proxies Reflecting Climate Change}

Fossil plant assemblages yield unambiguous signatures of past climate. This is because the zonal flora of a region is directly related to the macro-climate, and the regional floristic composition provides direct evidence on the vegetation type. Numerous studies indicate that morphological traits of fossil leaves provide information on climate, e.g., Bailey and Sinnott, 1916; Green and Hickey, 2005; Greenwood, 2005; Roth-Nebelsick et al., 2017; Royer, 2012; Wilf, 1997; Wolfe, 1993; Wolfe and Spicer, 1999, and that fossil taxa responded to climate (Roth-Nebelsick et al. 2021). The Climate Leaf Analysis Multivariate Program (CLAMP), which is based on morphological traits (Wolfe, 1993; Spicer, 2007), and the Coexistence Approach (CA), which is based on "nearest living relatives" (more precisely most similar relatives) (Mosbrugger and Utescher, 1997; Utescher et al., 2014), are current approaches to reconstruct climate from the plant fossil record. These reconstructions include mean annual temperature (MAT), warm-month mean temperature (WMMT), cold-month mean temperature (CMMT), and mean annual precipitation (MAP). The year-round distribution of precipitation also strongly influences the development of major vegetation types. This parameter is produced by CLAMP only (precipitation during the three consecutive wettest/driest months, 3-WET, 3-DRY). Summarising the climate proxy data based on these technologies reveals the overall cooling trend during the Palaeogene and Neogene mainly in the MAT and CMMT (Figure 8, Appendix 10).

The sequence of modern proxy sets of the test set of 54 fossil plant assemblages (Figure 3, Overall score) also reflects the global trend of decreasing temperature from the early Eocene to the Pleistocene as delineated most recently. In this sense, East Asian vegetation types and European vegetation formations develop from south to north (and from lower to higher altitudes) under climatic conditions with decreasing MAT. Asia "BLEF China, Japan" is characterised by the highest MAT, whereas the lowest MAT is characteristic of "MCF China, Japan". In Europe the warmest MAT occurs in the southernmost Formation $\mathrm{J}$ and the lowest ones in the northernmost Formation $\mathrm{C}$. Within the frame of this study MAT, MAP, WMMT, and CMMT have been assessed for the European vegetation Formations $\mathrm{F}, \mathrm{G}$, and $\mathrm{H}$ by the European Leaf Physiognomic Approach (ELPA) (Traiser and Mosbrugger, 2004) (Figure 9, Appendix 11). The results 


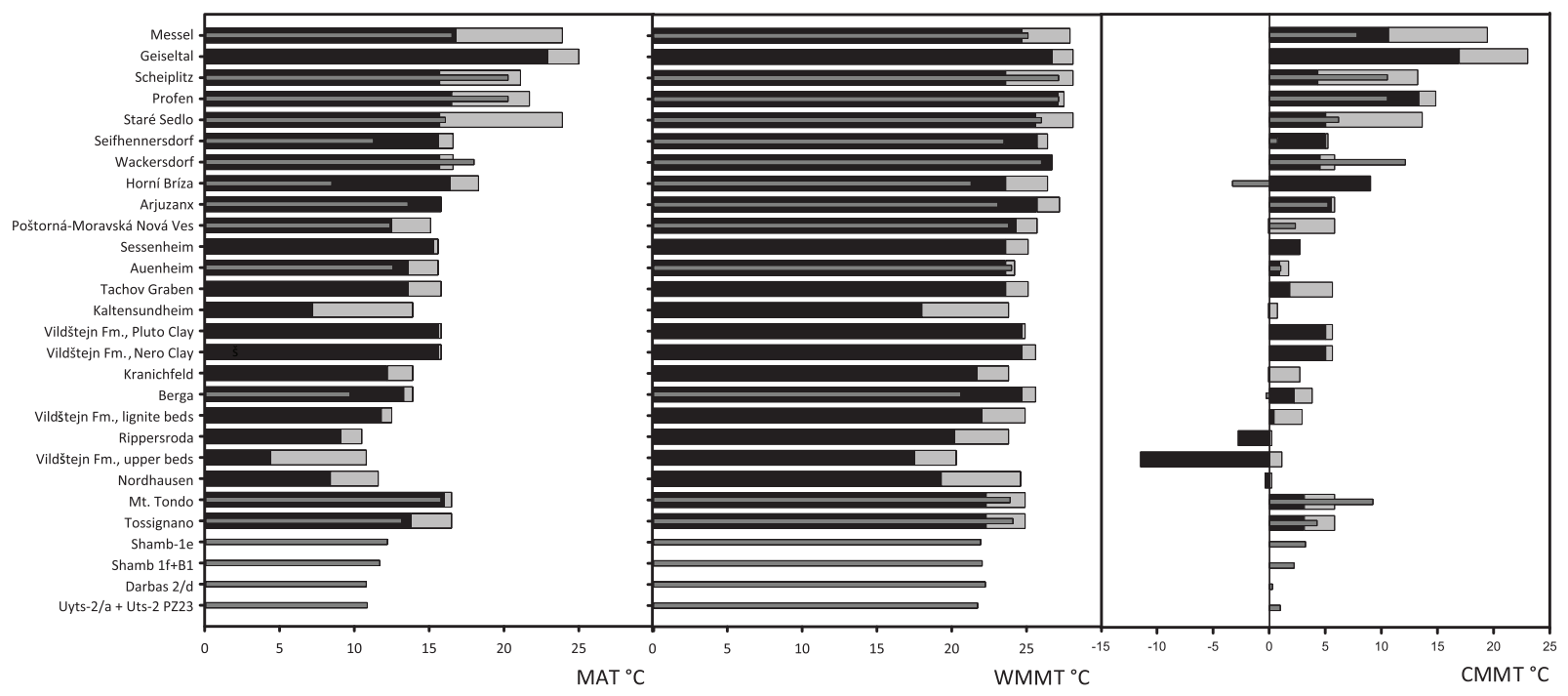

FIGURE 8. Mean annual temperature (MAT), warm-month mean temperature (WMMT), and cold-month mean temperature (CMMT) based on CLAMP and the Coexistence Approach (CA) for the fossil plant record (sources are Kvaček et al., 2011; Teodoridis and Kvaček, 2015; Teodoridis et al., 2009, 2012, 2015, 2017). black columns: minimum CA. light grey columns: maximum CA, narrow, dark grey columns: CLAMP result. For more comprehensive climate data see Appendix 10.

visualise a decrease of the temperature parameters MAT, CMMT, and WMMT from Formation $\mathrm{H}$ to Formation F. By trend MAP is lower in Formation $G$ than in Formation $\mathrm{H}$.

The stratigraphical resolution of the terrestrial plant record is usually lower than that of other sources, especially when it derives from nonmarine deposits. Nevertheless, some important climate excursions are well reflected by the modern vegetation proxies. The assemblages from the Hampshire Basin and London Clay record the period of the Early Eocene Climatic Optimum (EECO), a distinctive period characterised by elevated atmospheric $\mathrm{CO}_{2}$ and very warm temperatures (Zachos et al., 2001). The proxy sets of these assemblages are the only ones in the test set of fossil assemblages, which include China 01, Mount Emei - Evergreen Broad-leaved Forest Zone (660$1500 \mathrm{~m}$ alt.). The climate parameters in this region (Emeishan City) indicate a very warm and humid climate (MAT $17^{\circ} \mathrm{C}$, MAP $1528 \mathrm{~mm}$, WMMT 26.8 C, CMMT $7.1^{\circ} \mathrm{C}$; Teodoridis et al., 2011). The ECCO would possibly be even more strongly reflected by the proxies if the reference data set of modern vegetation included tropical vegetation from regions further south of China.

The early Oligocene cooling, which is related to the onset of Antarctic glaciation, is reflected by the assemblage of Seifhennersdorf in which, for the first time, the similarity is higher to proxies of "MMF China" and "BLDF Upper Yangtze, Honshu" than to "BLEF China, Japan".

The Middle Miocene Climate Optimum, which was a phase of globally warm and perhumid climatic conditions, is reflected in the test set of fossil assemblages by Oberdorf and Wackersdorf. These assemblages indicate the closest affinities to "BLEF China, Japan" of all the Miocene plant assemblages included here.

The middle Miocene is further characterised by the Middle Miocene Climatic Transition (MMCT) around $14 \mathrm{Ma}$, which was probably modulated by orbital forcing (Shevenell et al., 2004) and tectonic evolution, resulting in the reorganisation of ocean currents (Hamon et al., 2013). Isotope data indicate climate cooling (Zachos et al., 2001) and lower levels of atmospheric $\mathrm{CO}_{2}$ (Beerling and Royer, 2011). The signals delivered by modern vegetation proxies for Central European plant assemblages (Horní Bříza, Randeck Maar, Parschlug, Holy Cross Mts., Erdöbénye) differ markedly from those of older and younger plant assemblages. European vegetation formations are delivered more commonly as proxies, including the Formations $\mathrm{G}, \mathrm{H}, \mathrm{J}$, and $\mathrm{K}$, i.e., thermophilous and mixed deciduous broad-leaved forests, hygrophilous thermophytic mixed deciduous broad-leaved forests, mediterranean sclerophyllous forests and 


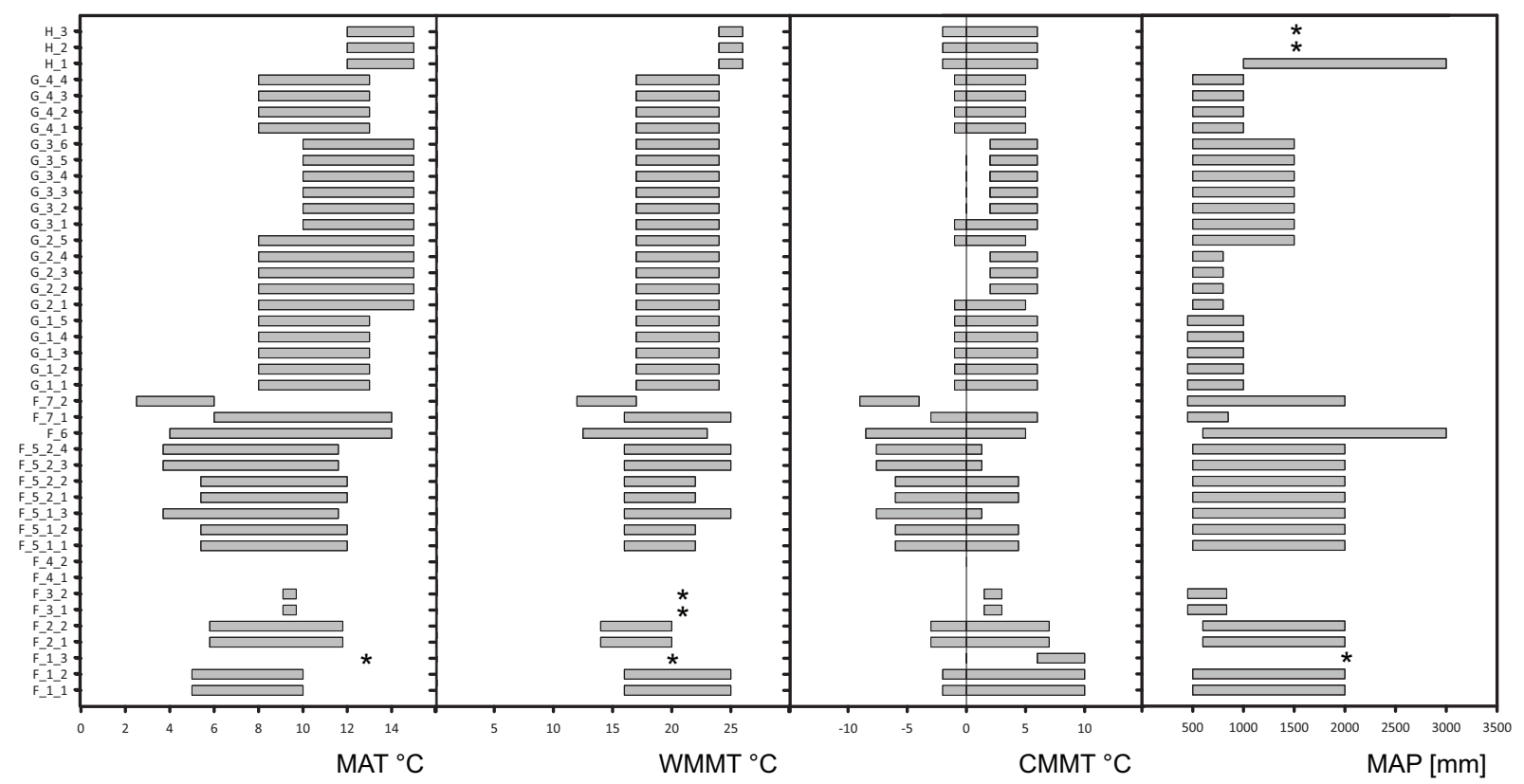

FIGURE 9. Climate parameters of the modern European vegetation Formations $F, G$, and $H$ based on Bohn et al. (2004) and Traiser and Mosbrugger (2004) represented as columns spanning the minimum and maximum of the respective data. Vegetation of Formation $F$ tends to lower temperatures (note, however, that climate data for formations F.3 - F.1 are more complex). Vegetation of Formation G tends to lower MAP. Asterisks indicate single data points (no climate interval was available). The data are listed in Appendix 11. Abbreviations: MAT = mean annual temperature; $\mathrm{WMMT}=$ warm-month mean temperature; $\mathrm{CMMT}=$ cold-month mean temperature; $\mathrm{MAP}=$ mean annual precipitation.

scrub, and xerophytic coniferous forests. This signal indicates more pronounced seasonality in precipitation than in preceding and later times. Lower MAP is indicated by the representation of Formation G, especially of subdivisions G.2 and G.3 (at lower elevations) (Figure 9, Appendix 11). The proxies allow to infer climatic conditions at the transition between Cfa (temperate, no dry season, hot summer)/Cfb (temperate, no dry season, warm summer) to Cs (temperate, summer-dry) climate sensu Köppen (Peel et al., 2007) (Kovar-Eder and Teodoridis, 2018). Unstable precipitation, although at the multiannual or decadal scale, was also proposed based on isotope signals of an estuarine oyster for the MMCT in Central Europe by Harzhauser et al. (2011). From the Yatagan Basin in SW Turkey, the climatic interpretation of the plant record based on leaf physiognomy (assessed by the CLAMP analysis, biogeographic affinities of the flora on the genus level, and "Köppen signatures") resulted in a Cf (temperate, no dry season) or possibly Cw (temperate, winter-dry) climate sensu Köppen there (Bouchal et al., 2018).
The isotope signatures of the mollusc fauna of Lake Pannon indicate climate change towards warm and perhumid conditions in the early late Miocene around $10 \mathrm{Ma}$ (Harzhauser et al., 2007). The flora of Mataschen at the westernmost extension of Lake Pannon may be related to this humid and warm phase. "BLEF China, Japan" appears again to a notable extent and ultimately as proxies in the Central European Miocene.

In the Central European record, the Plio-/ Pleistocene cooling is reflected by the increased relevance of "MCF China, Japan" and of the European Formation $F$ in the overall scores (Figures 3, $5,6)$. Furthermore, European vegetation proxies at that time gain increasing relevance compared to the late Miocene and early Pliocene.

\section{CONCLUSIONS}

The recently introduced Drudges 1 and 2 constitute automatised tools to assess modern vegetation proxies for Cenophytic plant assemblages. We applied the Drudges to 54 assemblages of leaves, fruits, seeds, and pollen from Europe, Turkey and 
Armenia ranging from the early Eocene to the early Pleistocene.

In the Palaeogene to the early Miocene the similarities (IPR Similarity, TS, Results Mix) consistently point towards a relationship to East Asian modern vegetation. From the early Miocene onwards the trends diverge, whereby the IPR Similarity points towards stronger European relationships than to East Asian ones, whereas TS still indicates closer ties to the East Asian flora. The weak representation of European vegetation proxies in TS can most likely be ascribed to the pauperisation of the European flora caused by the climatic oscillations during the Quaternary Ice Age.

The proxy sets for the early Eocene assemblages from Hampshire Basin and London Clay signal the Early Eocene Climatic Optimum in so far as they are the only ones including the modern vegetation of Mount Emei - Evergreen Broadleaved Forest Zone $(660-1500 \mathrm{~m}$ alt.), which is characterised by a very warm and humid climate.

The early Eocene to early Oligocene assemblages, which include the so-called "Older Mastixian floras" sensu Mai (1964), feature close similarity to "BLEF China, Japan", mainly to the BLEF of Longqi Mt. and to Broad-leaved Sclerophyllous Forests Taiwan, Fujian, and Guangxi sensu Wang (1961).

In contrast, Miocene and Pliocene assemblages with higher proportions of BLE taxa compared to other assemblages of this period, including the "Younger Mastixioid floras" sensu Mai indicate proxies mainly restricted to Broad-leaved Evergreen Sclerophyllous Forest Southern Hunan, the Southeastern Provinces, and Yunnan sensu Wang (1961) as well as to the BLEF of Mt. Fuji. Furthermore, for the "Younger Mastixioid floras" the Colchic mixed oak forests (sensu Bohn et al., 2004) are proxies.

Shared proxies between assemblages with high proportions of BLE taxa from the Palaeogene and Neogene are mainly Broad-leaved Evergreen Sclerophyllous Forests in the Maritime Provinces (Guangdong, Southern Hunan), in the Southeastern Provinces (Guizhou, Northern Guangxi) and Yunnan sensu Wang (1961).

For the early Oligocene site Seifhennersdorf, most of the proxies are part of the Mixed Mesophytic Forest Formation sensu Wang (1961), reflecting the early immigration of broad-leaved deciduous taxa often called "Arctotertiary" taxa. That immigration was likely linked to the global Early Oligocene Cooling.
For late early to middle Miocene assemblages the diversity of proxies is higher, reminiscent of the picture for the assemblages from the Miocene in southern Europe and Turkey. For the first time the European vegetation Formations D - Mesophytic and hygromesophytic coniferous and mixed broadleaved-coniferous forests, and F - Mesophytic broadleaved deciduous and mixed broadleaved/ conifer forests, gain importance as proxies. Formations $G$ - Thermophilous mixed deciduous broadleaved forests (G.2 to G.4), J - Mediterranean sclerophyllous forests and scrub, and $\mathrm{K}$ - Xerophytic coniferous forests (sensu Bohn et al., 2004) are more relevant than in the older and younger Central European record and Hyrcanian forests are delivered as proxies.

In Central Europe, proxies of the "MMF China", the "BLDF Upper Yangtze Provinces, Honshu", are more important for the late Miocene to Pliocene than for most of the middle Miocene assemblages. No proxies of the European Formations $\mathrm{J}, \mathrm{K}$, and $\mathrm{L}$ are delivered for the late Miocene to Pliocene and proxies of Formation $\mathrm{G}$ are rare.

For the Plio-/Pleistocene assemblages of Central Europe, proxies of the "MCF China, Japan" and of the European vegetation Formation F Mesophytic broadleaved deciduous and mixed broadleaved/conifer forests are more important than in earlier periods, whereas "MMF China" is least relevant. Within "MCF China, Japan", Montane Coniferous Forest - Picea obovata-Abies sibirica Forest (Mongolia 1) and Montane Coniferous Forest - Mixed Hardwoods (Mongolia 2) are tallied as proxies only for the Plio-/Pleistocene of Central Europe. Within Formation F, especially F.2 - Mixed oak-ash forests, F.3 - Mixed oak-hornbeam forests, and F.5 - Beech and mixed beech forests, are relevant. The Cantabrian-Euscaldian mixed oak-ash forests (unit F033, part of F.2) represent an essential proxy for the Plio-/Pleistocene Central European record, indicating a floristic relationship.

For the Miocene/Pliocene assemblages from the Mediterranean, proxies of East Asian vegetation are more important than European ones. The Armenian early Pleistocene record is distinguished by the higher number of European versus East Asian proxies in the overall scores. The TS, indicates, however, closer relationship to East Asia than to Europe. The Great Caucasus mountain ranges and the proximity of the Caspian Sea were potentially favourable for these ties. The Armenian assemblages differ further from the Central European Plio-/Pleistocene by the lesser importance of "MCF China, Japan", by the high relevance of For- 
mation $\mathrm{G}$ - Thermophilous mixed deciduous broadleaved forests, (especially G.1 to G.3) and by some proxies indicating Formation L - Forest steppes, dry grasslands.

The results, i.e., the modern proxies tallied for the fossil assemblages, reflect the overall vegetation evolution as well as the overall climate change from the Eocene to the Pleistocene. They also signal climate oscillations as the Early Eocene Climatic Optimum, the Early Oligocene Cooling, the Middle Miocene Climatic Optimum, the Middle Miocene Climatic Transition, and the climate deterioriation preceding the Pleistocene Ice Age. Future progress in stratigraphic and taxonomic resolution of fossil plant assemblages and the application of the Drudges will provide further differentiation of Cenophytic zonal vegetation in Europe.

\section{Glossary of terms}

Azonal vegetation. The development of plant communities is more strongly influenced by edaphic conditions than by climate, e.g., wetland or alluvial vegetation. Azonal taxa characterise azonal vegetation.

Overall score. All scores of all proxy sets (IPR Similarities, Results Mix of both Drudges and the Taxonomic Similarity (TS)) for each assemblage/ site; i.e., 25 proxies (five derived by each similarity approach). In this study the number of overall scores is 54 according to the number of sites.

Proxy. East Asian vegetation type or European vegetation formation or their subdivisions (units) as delivered by Drudge 1 and 2 .

Proxy set. The most closely corresponding five modern units derived by the Drudges for the IPR Similarities, the Results Mix of both Drudges, and the Taxonomic Similarity (TS).

Reference dataset of modern vegetation. Modern vegetation in Europe, the Caucasus, Mongolia, China, and Japan as categorised in Appendix 5.
Similarity approaches. IPR Similarity, Taxonomic Similarity (TS), Results Mix of Drudge 1 and 2, i.e., 5 approaches. Note, TS is identical in Drudge 1 and 2 and therefore is counted only once in the overall scores.

Test set of fossil assemblages. i.e., set of 54 fossil plant sites/assemblages in this study covering the early Eocene to Pleistocene.

Vegetation formations: Major categories of zonal vegetation in Europe as defined by Bohn et al. (2004), Table 3.

Vegetation types. Major categories of zonal vegetation in East Asia as defined in Table 3.

Vegetation units: Smallest scale subdivisions of East Asian vegetation types and European vegetation formations.

Sub-mediterranean. Transitional region between the Mediterranean region and the broad-leaved deciduous forest region (nemoral zone). The summer drought is less distinct than in the Mediterranean region.

Zonal vegetation. Large-scale vegetation developing under mesic soil conditions (no extremes). The given macro-climate is the determining factor for its performance. Zonal taxa mainly are bound to zonal vegetation.

\section{ACKNOWLEDGEMENTS}

The authors would like to thank I. Gabrielyan and A. Bruch for the floristic data from Armenia. We also thank the two anonymous reviewers for their stimulating comments. We dedicate this article to late Prof. Dr. Z. Kvaček, our teacher, colleague, and friend who influenced the development of the IPR vegetation analysis by his plant sociological experience. His taxonomic knowledge and research contributed to the taxonomic resolution of numerous fossil plant assemblages included here. This study was supported by the grant projects of GA ČR No. P18-25057S and Charles University, Prague, Progres Q16 (both VT).

\section{REFERENCES}

Andreánszky, A. 1959. Die Flora der Sarmatischen Stufe in Ungarn. Akadémia Kiadó, Budapest.

Bailey, I.W. and Sinnott, E.W. 1916. The climatic distribution of certain types of angiosperm leaves. American Journal of Botany, 3:24-39.

Beerling, D. and Royer, D. 2011. Convergent Cenozoic $\mathrm{CO}_{2}$ history. Nature Geoscience, 4:418420. https://doi.org/10.1038/ngeo1186 
Bertoldi, R. and Martinetto, E. 1995. Ricerche paleobotaniche (palinologiche e paleocarpologiche) sulla successione Villafranchiana del Rio Ca' Viettone (Torino, Italia). II Quaternario, 8:403-422.

Bohn, U., Neuhäusl, R., Gollub, G., Hettwer, C., Neuhäuslová, Z., Raus, Th., Schlüter, H., and Weber, H. 2004. Map of the Natural Vegetation of Europe. Maßstab / Scale 1:2500000. Landwirtschaftsverlag, Münster. Online available as EuroVegMap 2.06 http://www.floraweb.de/vegetation/dnld_eurovegmap.html

Bouchal, J.M., Güner, T.H., and Denk, T. 2018. Middle Miocene climate of southwestern Anatolia from multiple proxies. Climate of the Past, 14:1427-1440. https://doi.org/10.5194/cp-141427-2018

Bruch, A.A. and Gabrielyan, I.G. 2002. Quantifying Early Pleistocene climate and environmental change in Armenia. Acta Universitatis Carolinae Geologica, 46(4):41-48.

Bůžek, C., Kvaček, Z., and Holý, F. 1985. Late Pliocene palaeoenvironment and correlation of the Vildstejn floristic complex within Central Europe. Rozpravy Československé Akademie věd. Řada matematických a přirodních věd, 95(7):1-72.

Collinson, M.E. 1983. Fossil Plants of the London Clay. Palaeontological Association Field Guides to Fossils, 1. Palaeontological Association, London.

Collinson, M., Kvaček, Z., and Zastawniak, E. 2001. The aquatic plants Salvinia (Salviniales) and Limnobiophyllum (Arales) from the Late Miocene flora of Sosnica (Poland). Acta Palaeobotanica, 41:253-282.

Collinson, M.E., Manchester, R.S., and Wilde, V. 2012. Fossil fruits and seeds of the Middle Eocene Messel biota, Germany. Abhandlungen Senckenberg Gesellschaft für Naturforschung, 570:1-251.

Denk, T., Güner, T.H., Kvaček, Z., and Bouchal, J.M. 2017. The early Miocene flora of Güvem (Central Anatolia, Turkey): a window into early Neogene vegetation and environments in the Eastern Mediterranean. Acta Palaeobotanica, 57:237-338. https://doi.org/10.1515/acpa2017-0011

Dyjor, S., Sadowska, A., Hummel, A., Baranowska-Zarzycka, E., and Zastawniak, E. 1998. Geological position and flora of the younger Neogene deposits in Lower Silesia, p. 37-39. In Sadowska, A. and Szynkiewicz, A. (eds.), Guide to Excursion 2, Tertiary-Quaternary (Pleistocene) floras of Bełchatów (Middle Poland) and several localities in south-western Poland. The $5^{\text {th }}$ European Palaeobotanical and Palynological Conference, June 26-30, 1998, Cracow, Poland. Władysław Szafer Institute of Botany, Polish Academy of Sciences, Kraków.

Erdei, B. and Hír, J. 2002. Vegetation and climate reconstruction of Sarmatian (Middle Miocene) sites from NE and W Hungary. Acta Universitatis Carolinae, Geologica, 46(4):75-84.

Fischer, O. 1991. Blätter-Floren aus mitteleozänen Sedimenten des südlichen Weißelster Beckens (Profen und Scheiplitz). Unpublished PhD Thesis, Humboldt-Universität Berlin, Museum für Naturkunde, Berlin.

Goeppert, H.R. 1855. Die tertiäre Flora von Schossnitz in Schlesien. Heynische Buchhandlung (E. Remer), Görlitz.

Green, W.A. and Hickey, L.J. 2005. Leaf architectural profiles of angiosperm floras across the Cretaceous/Tertiary boundary. American Journal of Science, 305:983-1013. https://doi.org/ 10.2475/ajs.305.10.983

Greenwood, D.R. 2005. Leaf margin analysis: taphonomic constraints. Palaois, 20:498-505. https://doi.org/10.2110/palo.2004.P04-58

Gregor, H.-J. 1978. Die miozänen Frucht-und Samenfloren der Oberpfälzer Braunkohle. I. Funde aus den sandigen Zwischenmitteln. Palaeontographica, Abteilung B, 167:9-103.

Güner, T.H., Bouchal, J.M., Köse, N., Goktas, F., Mayda, S., and Denk, T. 2017. Landscape heterogenity in the Yatagan Basin (southwestern Turkey) during the middle Miocene inferred from plant macrofossils. Palaeontographica, Abteilung B, 296:113-171. https://doi.org/ 10.1127/palb/296/2017/113

Günther, T. and Gregor, H.-J. 1989. Computeranalyse neogener Frucht-und Samenfloren Europas. Band 1: Fundorte und deren Florenlisten. Documenta naturae, 50(1):1-180.

Günther, T. and Gregor, H.-J. 1993. Computeranalyse neogener Frucht-und Samenfloren Europas. Documenta naturae, 50(4):1-190.

Hably, L. 2020. The Karpatian (late early Miocene) flora of the Mecsek area. Acta Palaeobotanica, 60:51-122. https://doi.org/10.35535/acpa-2020-0003 
Hamon, N., Sepluchre, P., Lefebvre, V., and Ramstein, G. 2013. The role of eastern Tethys seaway closure in the Middle Miocene Climatic Transition (ca. $14 \mathrm{Ma}$ ). Climate of the Past, 9:2687-2702. https://doi.org/10.5194/cp-9-2687-2013

Harzhauser, M., Latal, C., and Piller, W. 2007. The stable isotope archive of Lake Pannon as a mirror of Late Miocene climate change. Palaeogeography, Palaeoclimatology, Palaeoecology, 249:335-350. https://doi.org/10.1016/j.palaeo.2007.02.006

Harzhauser, M., Piller, W.E., Müllegger, S., Grunert, P., and Micheels, A. 2011. Changing seasonality patterns in Central Europe from the Miocene Climate Optimum to Miocene Climate Transition deduced from the Crassostrea isotope archive. Global and Planetary Change, 76:77-84. https://doi.org/10.1016/j.gloplacha.2010.12.003

Hummel, A. 1983. The Pliocene leaf flora from Ruszow near Zary in Lower Silesia, SW Poland. Prace Muzeum Ziemi, 36:9-96.

Hummel, A. 1991. The Pliocene leaf flora from Ruszow near Zary in Lower Silesia, South-West Poland. Part 2 (Betulaceae). Acta Palaeobotanica, 31:73-151.

Kahlert, E. and Rüffle, L. 2007. Leguminosenblätter des Geiseltales (Eozän, Sachsen-Anhalt) und ihre Beziehungen zum Alttertiär Nordamerikas. Documenta naturae, 166:1-36.

Knobloch, E. 1969. Tertiäre Floren vom Mähren. Moravské Museum, Brno.

Knobloch, E. and Kvaček, Z. 1976. Miozäne Blätterfloren vom Westrand der Böhmischen Masse. Rozpravy Ústředního Ústavu Geologického, 42:5-129.

Knobloch, E., Kvaček, Z., and Konzalova, M. 1996. Die obereozäne Flora der Staré SedloSchichtenfolge in Böhmen (Mitteleuropa). Rozpravy Českého Geologického Ústavu, 49:1260.

Knörr, U.C., Kovar-Eder, J., Mazouch, P., and Roth-Nebelsick, A. 2012. Fruit dispersal ecology of woody taxa in temperate to tropical forests of China and Japan. Palaios, 27:523-540. https:// doi.org/10.2110/palo.2011.p11-113r

Kohlman-Adamska, A., Ziembinska-Tworzydlo, M., and Zastawniak, E. 2004. In situ pollen in some flowers and inflorescences in the Late Miocene flora of Sosnica (SW Poland). Review of Palaeobotany and Palynology, 132:261-280. https://doi.org/10.1016/ j.revpalbo.2004.07.005

Kovar, J. 1982. Eine Blätter-Flora des Egerien (Ober-Oligozän) aus marinen Sedimenten der Zentralen Paratethys im Linzer Raum (Österreich). Beiträge zur Paläontologie in Österreich, 9:1-209.

Kovar-Eder, J. 1988. Obermiozäne (Pannone) Floren aus der Molassezone Österreichs. Beiträge zur Paläontologie in Österreich, 14:19-121.

Kovar-Eder, J. 2016. Early Oligocene plant diversity along the Upper Rhine Graben: the fossil flora of Rauenberg, Germany. Acta Palaeobotanica, 56:329-440. https://doi.org/10.1515/acpa-2016-0011

Kovar-Eder, J., Kvaček, Z., and Meller, B. 2001. Comparing Early to Middle Miocene floras and probable vegetation types of Oberdorf N Voitsberg (Austria), Bohemia (Czech Republic), and Wackersdorf (Germany). Review of Palaeobotany and Palynology, 114:83-125. https:// doi.org/10.1016/s0034-6667(00)00070-1

Kovar-Eder, J., Kvaček, Z., and Ströbitzer-Hermann, M. 2004. The Miocene flora of Parschlug (Styria, Austria) - revision and synthesis. Annalen des Naturhistorischen Museums Wien, 105A:45-159.

Kovar-Eder, J. and Hably, L. 2006. The flora of Mataschen - a unique plant assemblage from the late Miocene of eastern Styria (Austria). Acta Palaeobotanica, 46:157-233.

Kovar-Eder, J. and Kvaček, Z. 2007. The integrated plant record (IPR) to reconstruct Neogene vegetation - the IPR vegetation analysis. Acta Palaeobotanica, 47:391-418.

Kovar-Eder, J., Jechorek, H., Kvaček, Z., and Parashiv, V. 2008. The integrated plant record: an essential tool to reconstruct Neogene zonal vegetation in Europe. Palaios, 23:97-111. https:// doi.org/10.2110/palo.2006.p06-039r

Kovar-Eder, J., Knörr, U.C., and Mazouch, P. 2013. Fruit ecology of Eocene and Neogene plant assemblages in Europe: tracing shifts in dispersal syndromes. Palaios, 27:887-903. https://doi.org/10.2110/palo.2012.p12-051r

Kovar-Eder, J. and Teodoridis, V. 2018. The Middle Miocene Central European plant record revisited; widespread subhumid sclerophyllous forests indicated. Fossil Imprint, 74:115-134. https://doi.org/10.2478/if-2018-0009

Kóvats, J. 1856a. Fossile Flora von Erdöbenye. Arbeiten der geologischen Gesellschaft für Ungarn, 1:1-37. 
Kóvats, J. 1856b. Fossile Flora von Tállya. Arbeiten der geologischen Gesellschaft für Ungarn, 1:39-52.

Kvaček, Z. 2004. Revisions to the Early Oligocene flora of Flörsheim (Mainz Basin, Germany) based on epidermal anatomy. Senckenbergiana Lethaea, 84(1-2):1-73. https://doi.org/ $10.1007 /$ bf03043465

Kvaček, Z., Teodoridis, V., and Gregor, H.-J. 2008. The Pliocene leaf flora of Auenheim, Northern Alsace (France). Documenta naturae, 155(10):1-108.

Kvaček, Z., Teodoridis, V., and Roiron, P. 2011. A forgotten Miocene mastixioid flora of Arjuzanx. Palaeontographica, Abteilung B, 285:3-111. https://doi.org/10.1127/palb/285/2011/3

Łańcucka-Środoniowa, M. 1966. Tortonian flora from the "Gdów Bay" in the south of Poland. Acta Palaeobotanica, 7:1-135.

Łańcucka-Środoniowa, M. 1984. The results obtained hitherto in studies on the Miocene macroflora from the salt-mine at Wieliczka (S.Poland). Acta Palaeobotanica, 24:3-26.

Łańcucka-Środoniowa, M. and Zastawniak, E. 1997. The middle Miocene flora of Wieliczka revision of Jan Zablocki's collection. Acta Palaeobotanica, 37:17-49.

Mai, D.H. 1964. Die Mastixioideen-Floren im Tertiär der Oberlausitz. Paläontologische Abhandlungen, Abteilung B, 2(1):1-192.

Mai, D.H. 1976. Fossile Früchte und Samen aus dem Mitteleozän des Geiseltales. Abhandlungen des Zentralen Geologischen Instituts, 26:93-149.

Mai, D.H. and Walther, H. 1988. Die pliozänen Floren von Thüringen/Deutsche Demokratische Republik. Quartärpaläontologie, 7:55-297.

Mai, D.H. and Walther, H. 2000. Die Fundstellen eozäner Floren des Weisselster-Beckens und seiner Randgebiete. Altenburger Naturwissenschaftliche Forschungen, 13:1-59.

Manchester, S.R. and Zastawniak, E. 2007. Fruit with perianth remains of Chaneya Wang \& Manchester (extinct Rutaceae) in the Upper Miocene of Sośnica, Poland. Acta Palaeobotanica, 47:253-259.

Martinetto, E. and Vassio, E. 2010. Reconstructing "Plant Community Scenarios" by means of carpological data from the CENOFITA database, with an example from the Ca'Viettone site (Pliocene, Northern Italy). Quaternary International, 225:25-36. https://doi.org/10.1016/ j.quaint.2009.08.020

Mosbrugger, V. and Utescher, T. 1997. The coexistence approach-a method for quantitative reconstructions of Tertiary terrestrial palaeoclimate data using plant fossils. Palaeogeography, Palaeoclimatology, Palaeoecology, 134:61-86. https://doi.org/10.1016/ s0031-0182(96)00154-x

Němejc, F., Kvaček, Z., Pacltová, B., and Konzalová, M. 2003. Tertiary plants of the Plzeň Basin (West Bohemia). Acta Universitatis Carolinae, Geologica, 46(2002):121-176.

Peel, M.C., Finlayson, B.L., and Mcmahon, T.A. 2007. Updated world map of Köppen-Geiger climate classification. Hydrology and Earth System Sciences Discussions, European Geosciences Union, 11(5):1633-1644.

Rasser, M.W., Bechly, G., Böttcher, R., Ebner, M., Heizmann, E.P.J., Höltke, O., Joachim, C., Kern, A.K., Kovar-Eder, J., Nebelsick, J.H., Roth-Nebelsick, A., Schoch, R.R., Schweigert, G., and Ziegler, R. 2013. The Randeck Maar: palaeoenvironment and habitat differentation of a Miocene lacustrine system. Palaeogeography, Palaeoclimatology, Palaeoecology, 392:426-453. https://doi.org/10.1016/j.palaeo.2013.09.025

Roth-Nebelsick, A., Grein, M., Traiser, C., Moraweck, K., Kunzmann, L., Kovar-Eder, J., Kvaček, J., Stiller, S., and Neinhuis, C. 2017. Functional leaf traits and leaf economics in the Paleogene - a case study for Central Europe. Palaeogeography, Palaeoclimatology, Palaeoecology, 472:1-14. https://doi.org/10.1016/j.palaeo.2017.02.008

Roth-Nebelsick, A. Grein, M., Traiser, C., Kunzmann, L., Kvaček, J., Wypich, J., and KovarEder. J. 2021. Taxon-specific variability of leaf traits in three long-ranging fossil taxa of the Paleogene and Neogene: responses to climate? Palaeontologia Electronica, 24(1):a04. https://doi.org/10.26879/1114

Royer. D.L. 2012. Climate reconstruction from leaf size and shape: new developments and challenges. The Paleontological Society Papers, 18:195-212. https://doi.org/10.1017/s1089332600002618

Rüffle, L. 1963. Die obermiozäne (sarmatische) Flora vom Randecker Maar. Paläontologische Abhandlungen, 1(3):139-298. 
Shevenell, A., Kennett, J.P., and Lea, D.W. 2004. Middle Miocene Southern ocean cooling and Antarctic cryosphere expansion. Science, 305:1766-1769. https://doi.org/10.1126/ science.1100061

Spicer, R.A. 2007. Recent and future developments of CLAMP: building on the legacy of Jack A. Wolfe. Courier Forschungsinstitut Senckenberg, 258:109-118.

Stachurska, A., Sadowska, A., and Dyjor, S. 1973. The Neogene flora at Sosnica near Wroclaw in the light of geological and palynological investigations. Acta Palaeobotanica, 14:147-176.

Striegler, U. 2008. Pflanzenfossilien aus den eemzeitlichen sowie spätsaalzeitlichen und frühweichselzeitlichen Schichten von Klinge bei Cottbus. Natur und Landschaft in der Niederlausitz, 27:16-71.

Stuchlik, L. 1982. Rostlinné mikrofosílie vildštejnského souvrství (chebská pánev). Časopis pro Mineralogii a. Geologii, 27(3):301-307.

Sturm, M. 1971. Die eozäne Flora von Messel bei Darmstadt. I. Lauraceae. Palaeontographica, Abteilung B, 134(1-3):1-60.

Szafer, W. 1961. Mioceńska flora ze starych Gliwic na Śląsku. Instytut Geologiczny Prace, 33:1205.

Teodoridis, V., Kvaček, Z., and Uhl, D. 2009. Pliocene palaeoenvironment and correlation of the Sessenheim-Auenheim floristic complex (Alsace, France). Palaeodiversity, 2:1-17.

Teodoridis, V., Kovar-Eder, J., and Mazouch, P. 2011. Integrated Plant Record (IPR) vegetation analysis applied to modern vegetation in South China and Japan. Palaios, 26:623-638. https://doi.org/10.2110/palo.2010.p10-149r

Teodoridis, V., Kvaček, Z., Zhu, H., and Mazouch, P. 2012. Environmental analysis of the midlatitudinal European Eocene sites of plant macrofossils and their possible analogues in East Asia. Palaeogeography, Palaeoclimatology, Palaeoecology, 333-334:40-58. https://doi.org/ 10.1016/j.palaeo.2012.03.008

Teodoridis, V. and Kvaček, Z. 2015. Palaeoenvironmental evaluation of Cainozoic plant assemblages from the Bohemian Massif (Czech Republic) and adjacent Germany. Bulletin of Geosciences, 90(3):695-720. https://doi.org/10.3140/bull.geosci.1553

Teodoridis, V., Kvaček, Z., Sami, M. Utescher, T., and Martinetto, E. 2015. Palaeoenvironmental analysis of the Messinian macrofossil floras of Tossignano and Monte Tondo (Vena del Gesso Basin, Romagna Apennines, Northern Italy). Sborník Národního muzea v Praze, řada B, 71(3-4):249-292.

Teodoridis, V., Bruch, A.A., Vassio, E., Martinetto, E., Kvaček, Z., and Stuchlik, L. 2017. PlioPleistocene floras of the Vildštejn Formation in the Cheb Basin, Czech Republic - a floristic and palaeoenvironmental review. Palaeogeography, Palaeoclimatology, Palaeoecology, 467:66-190. https://doi.org/10.1016/j.palaeo.2015.09.038

Teodoridis, V., Mazouch, P., and Kovar-Eder, J. 2020. The Integrated Plant Record (IPR) analysis: methodological advances and new insights into the evolution of European Palaeogene/Neogene vegetation. Palaeontologia Electronica, 23(1):a16. https://doi.org/ 10.26879/1055

Teodoridis, V., Kovar-Eder, J., Marek, P., Mazouch, P., and Kvaček, Z. 2011-2021. IPR database. http://www.iprdatabase.eu/. Faculty of Education, Charles University, Prague.

Teodoridis, V., Mazouch, P., and Kovar-Eder, J. 2021. On-line application of Drudge 1 and 2 simple and quick determination of the modern vegetation most closely resembling fossil plant assemblages. Neues Jahrbuch für Geologie und Paläontologie Abhandlungen, 299(1):1-5. https://doi.org/10.1127/njgpa/2021/0955

Traiser, C. and Mosbrugger, V. 2004. ELPA (European Leaf Physiognomic Approach): grid data set of environmental and ecological parameters. PANGAEA, https://doi.org/10.1594/ PANGAEA.218187

Utescher, T., Erdei, B., François, L., and Mosbrugger, V. 2007. Tree diversity in the Miocene forests of Western Eurasia. Palaeogeography, Palaeoclimatology, Palaeoecology, 253:226250. https://doi.org/10.1016/j.palaeo.2007.03.041

Utescher, T., Bruch, A.A., Erdei, B., François, L., Ivanov, D., Jacques, F.M.B., Kern, A.K., Liu, Y.S., Mosbrugger, V., and Spicer, R.A. 2014. The Coexistence Approach - theoretical background and practical considerations of using plant fossils for climate quantification. Palaeogeography, Palaeoclimatology, Palaeoecology, 410:58-73. https://doi.org/10.1016/ j.palaeo.2014.05.031

Velitzelos, D. (ed.) 2002. Field Trip Guidebook Evia Island, Neogene Sedimentary Basin of Kymi/ Aliveri. Athens. 
Walther, H. and Zastawniak, E. 1991. Fagaceae from Sosnica and Malczyce (near Wroclaw, Poland). A revision of original materials by Goeppert 1852 and 1855 and a study of new collections. Acta Palaeobotanica, 31:153-199.

Walther, H. and Kvaček, Z. 2007. Early Oligocene flora of Seifhennersdorf (Saxony). Sborník Národního muzea v Praze, řada B, 63:85-174.

Wang, C.-W. 1961. The forests of China with a survey of grassland and desert vegetation. Maria Moors Cabot Foundation, 5:1-313.

Wilde, V. 1989. Untersuchungen zur Systematik der Blattreste aus dem Mitteleozän der Grube Messel bei Darmstadt (Hessen, Bundesrepublik Deutschland). Courier Forschungsinstitut Senckenberg, 115:1-215.

Wilde, V. 1995. Die Makroflora aus dem Mitteleozän des Geiseltalgebietes, kurze Übersicht und Vergleiche. Hallesches Jahrbuch der Geowissenschaften, B, 17:121-138.

Wilde, V., Kvaček, Z., and Bogner, J. 2005. Fossil leaves of the Araceae from the Eocene of Europe. International Journal of Plant Sciences, 166:157-183. https://doi.org/10.1086/ 425673

Wilf, P. 1997. When are leaves good thermometers? A new case for Leaf Margin Analysis. Paleobiology, 23:373-390. https://doi.org/10.1017/S0094837300019746

Wolfe, J.A. 1993. A method of obtaining climatic parameters from leaf assemblages. United States Geological Survey Bulletin, 2040:1-73.

Wolfe, J.A. and Spicer, R.A. 1999. Fossil leaf character states: multivariate analysis, p. 233-239. In Jones, T.P. and Rowe, N.P. (eds.), Fossil Plants and Spores: Modern Techniques. Geological Society, London.

Zachos, J., Pagani, M., Sloan, L., Thomas, E., and Billups, K. 2001. Trends, rhythms, and aberrations in global climates 65 Ma to present. Science, 292:686-693. https://doi.org/ 10.1126/science.1059412

Zastawniak, E. 1980. Sarmatian leaf flora from the southern margin of the Holy Cross Mts. (South Poland). Prace Museum Ziemi, 33:39-107.

Zastawniak, E. and Walther, H. 1998. Betulaceae from Sosnica near Wroclaw (Poland) - a revision of Goeppert's original materials and a study of more recent collections. Acta Palaeobotanica, 38:87-145.

Zidianakis, G., Iliopoulos, G., Zelilidis, A., and Kovar-Eder, J. 2015. Myrica from the plant assemblage of Pitsidia (Crete, Late Miocene): putting the puzzle together. Palaeontographica, Abteilung B, 293:149-171.

Zidianakis, G., Iliopoulos, G., Zelilidis, A., and Kovar-Eder, J. 2016. Pinus remains from the Pitsidia plant assemblage document costal pine forests in southern Crete during the Late Miocene. Review of Palaeobotany and Palynology, 235:11-30. https://doi.org/10.1016/ j.revpalbo.2016.09.003

Zidianakis, G., Iliopoulos, G., Zelilidis, A., and Kovar-Eder, J. 2020. Three middle to late Miocene plant macro-remain assemblages (Pitsidia, Kassanoi and Metochia) from the Messara Gavdos region, southern Crete. Acta Palaeobotanica, 60:333-437. https://doi.org/10.35535/ acpa-2020-0018 


\section{APPENDIX 1.}

IPR scores of the 54 early Eocene to Pleistocene plant assemblages from Europe, Turkey and Armenia (test set of fossil assemblages) included in this study. (All appendices are available in a downloadable zipped file https://palaeo-electronica.org/content/2021/3352-vegetation-evolutionand-climate-change.)

\section{APPENDIX 2.}

Copy and paste application of Drudge 1 including taxa, IPR scoring, and results for the early Oligocene plant assemblage of Seifhennersdorf (Germany) version Drudge_1_505_corr_20200510. (All appendices are available in a downloadable zipped file https://palaeoelectronica.org/content/2021/3352-vegetation-evolution-and-climate-change.)

\section{APPENDIX 3.}

Copy and paste application of Drudge 2 including taxa, IPR scoring, and results for the early Oligocene plant assemblage of Seifhennersdorf (Germany) version Drudge_2_505_corr_20200510.(All appendices are available in a downloadable zipped file https://palaeoelectronica.org/content/2021/3352-vegetation-evolution-and-climate-change.)

\section{APPENDIX 4.}

Matrix resulting from the application of Drudges 1 and 2 transferred to a spreadsheet and visualisation of the results with interactive colour signature. The fossil assemblages (abscissa) are plotted against the dataset of modern vegetation types and their units (ordinate). Only those units are included that received scores. The five best fitted results are mapped for IPR Similarity, Taxonomic Similarity (TS), and Results Mix for both Drudges as well as the synthesis of all results derived by both Drudges (sheet "Overall scores Drudge 1+2"). Note that TS is mapped only once in the overall scores because it is identical for Drudge 1 and 2. Moving the cursor over the columns provides the designation of the proxies and their relevance for every fossil assemblage. (All appendices are available in a downloadable zipped file https://palaeo-electronica.org/ content/2021/3352-vegetation-evolution-and-climate-change.)

\section{APPENDIX 5.}

Vegetation units from Europe and the Caucasus (Bohn et al., 2004), China and Mongolia (Wang, 1961), China and Japan (Teodoridis et al., 2011, Teodoridis et al., 2012). Linkage of ID codes and the designation of the modern plant units (from Teodoridis et al., 2020, app. 7). (All appendices are available in a downloadable zipped file https://palaeo-electronica.org/content/2021/3352vegetation-evolution-and-climate-change.)

\section{APPENDIX 6.}

Results of the Integrated Plant Record (IPR) vegetation analysis for modern vegetation proxies in East Asia and Europe showing the proportions of the major zonal woody angiosperm components BLD (broad-leaved deciduous), BLE (broad-leaved evergreen), SCL+LEG (sclerophyllous plus legume-like). Included are only those units that were delivered as proxies by applying the Drudges. The complete score results of the IPR vegetation analysis are available in Teodoridis et al. (2020, appendices 1-4). (All appendices are available in a downloadable zipped file https:// palaeo-electronica.org/content/2021/3352-vegetation-evolution-and-climate-change.) 


\section{APPENDIX 7.}

Comparison of East Asian forest types, mixed mesophytic forests (MMF), broad-leaved deciduous forests (BLDF), mountain coniferous forests (MCF), regarding diversity and proportion of major zonal woody angiosperm components based on the IPR vegetation analysis performed on the data provided by Wang (1961). The detailed scores are available in Teodoridis et al. (2020, appendix 4) and Teodoridis et al. (2011-2021). (All appendices are available in a downloadable zipped file https://palaeo-electronica.org/content/2021/3352-vegetation-evolution-and-climatechange.)

\section{APPENDIX 8.}

Relevance of modern East Asian and European vegetation types and formations as derived by Drudge 1 and 2 in the IPR Similarities, Taxonomical Similarity (TS), and Results Mix. (All appendices are available in a downloadable zipped file https://palaeo-electronica.org/content/2021/ 3352-vegetation-evolution-and-climate-change.)

\section{APPENDIX 9.}

Relevance of modern East Asian and European vegetation types and formations regarding age and region as derived by Drudge 1 and 2. (All appendices are available in a downloadable zipped file https://palaeo-electronica.org/content/2021/3352-vegetation-evolution-and-climatechange.)

\section{APPENDIX 10.}

Climate data for fossil sites reconstructed by CLAMP and the Coexistence Approach. (All appendices are available in a downloadable zipped file https://palaeo-electronica.org/content/2021/ 3352-vegetation-evolution-and-climate-change.)

\section{APPENDIX 11.}

Climatic parameters of the modern European vegetation Formations $\mathrm{F}, \mathrm{G}$, and $\mathrm{H}$. Climate data marked with an asterisk: obtained from Bohn et al. (2004). Data marked with "^" obtained from Traiser and Mosbrugger (2004). Abbreviations: MAT = mean annual temperature; WMMT = warm-month mean temperature; $\mathrm{CMMT}=$ cold-month mean temperature; $\mathrm{MAP}=$ mean annual precipitation; $\mathrm{N}=$ North, $\mathrm{E}=$ East, $\mathrm{S}=$ South, $\mathrm{W}=$ West. (All appendices are available in a downloadable zipped file https://palaeo-electronica.org/content/2021/3352-vegetation-evolution-andclimate-change.) 